Florida International University FIU Digital Commons

FIU Electronic Theses and Dissertations

University Graduate School

$11-9-2010$

\title{
An Improved Framework for Dynamic Origin- Destination (O-D) Matrix Estimation
}

Hongbo Chi

Florida International University, hchi001@fiu.edu

DOI: $10.25148 /$ etd.FI10120309

Follow this and additional works at: https://digitalcommons.fiu.edu/etd

Part of the Civil Engineering Commons

\section{Recommended Citation}

Chi, Hongbo, "An Improved Framework for Dynamic Origin-Destination (O-D) Matrix Estimation" (2010). FIU Electronic Theses and Dissertations. 289.

https://digitalcommons.fiu.edu/etd/289

This work is brought to you for free and open access by the University Graduate School at FIU Digital Commons. It has been accepted for inclusion in FIU Electronic Theses and Dissertations by an authorized administrator of FIU Digital Commons. For more information, please contact dcc@fiu.edu. 


\section{FLORIDA INTERNATIONAL UNIVERSITY}

Miami, Florida

\section{AN IMPROVED FRAMEWORK FOR DYNAMIC ORIGIN-DESTINATION (O-D) \\ MATRIX ESTIMATION}

A dissertation submitted in partial fulfillment of the

requirements for the degree of

DOCTOR OF PHILOSOPHY

in

CIVIL ENGINEERING

by

Hongbo Chi 
To: Dean Amir Mirmiran

College of Engineering and Computing

This dissertation, written by Hongbo Chi, and entitled An Improved Framework for Dynamic Origin-Destination (O-D) Matrix Estimation, having been approved in respect to style and intellectual content, is referred to you for judgment.

We have read this dissertation and recommend that it be approved.

Zhenmin Chen

Albert Gan

Mohammed Hadi, Co-Major Professor

Fang Zhao, Co-Major Professor

Date of Defense: November 9, 2010

The dissertation of Hongbo Chi is approved.

Dean Amir Mirmiran

College of Engineering and Computing

Interim Dean Kevin O'Shea

University Graduate School

Florida International University, 2010 


\section{DEDICATION}

I dedicate this dissertation to my parents, Guihua Li and Jianzhong Chi, and to my elder sister Hongyan Chi, for their unconditional and endless love. 


\section{ACKNOWLEDGMENTS}

Above all, I would like to thank my major advisor, Dr. Fang Zhao, not only for her inspiration, guidance, and support during my doctoral life, but also for her patience, tolerance, and confidence in me. In my opinion, she exemplifies a responsible professional in our society. She is my mentor as well as my friend.

I would also like to thank my Co-Major Advisor, Dr. Hadi, who is experienced in the transportation field and shared many good ideas with me. In addition, he is also a very generous professor towards his students. I also feel very grateful to the rest of my dissertation committee members and other professors for their guidance. In particular, Dr. Albert Gan is a strict, but friendly, professor. In doing everything professionally, he has proven to be a respectable person. I took many statistic classes from Dr. Zhenming Chen. More than a statistics expert, he always finds a way to explain difficult concepts in understandable ways. He provided me with very useful guidance in my research. I would also like to thank Dr. L. David Shen, who taught my first class upon arrival in America. Dr. Shen enlightened me on my future points of study and career.

I want to thank my colleagues for collaborating with me all of these years at FIU, specifically Dr. Mintang Li, Dr. Lee-Fang Chow, Dr. Soon Chung, Dr. Xuemei Liu, Dr. Zhen Ding, Dr. Jing Qi, Shanshan Yang, Dr. Chenxi Lu, and Dr. Keqiang Xing. I am also grateful to Dr. Halit Ozen, Yan Xiao, Huijing Qiang, and Dr. Wangyang Wu. Additionally, Ms. Amy Diaz, Ms. Lilia Silverio, Ms. Laura Osorno, and Ms. Haydee Cadena provided many helpful services to the students in the college and the department, and I am grateful for their help in my overseas study. 
I would like to acknowledge the financial support from Florida International University. The presidential enhanced assistantship and the dissertation year fellowship awarded by the university graduate school offered me strong support during my years of study.

Finally, I would like to thank all of my friends for their care and support during my study overseas. 


\section{ABSTRACT OF THE DISSERTATION \\ AN IMPROVED FRAMEWORK FOR DYNAMIC ORIGIN-DESTINATION (O-D) \\ MATRIX ESTIMATION}

by

Hongbo Chi

Florida International University, 2010

Miami, Florida

\section{Professor Mohammed Hadi, Co-Major Professor \\ Professor Fang Zhao, Co-Major Professor}

This dissertation aims to improve the performance of existing assignment-based dynamic origin-destination (O-D) matrix estimation models to successfully apply Intelligent Transportation Systems (ITS) strategies for the purposes of traffic congestion relief and dynamic traffic assignment (DTA) in transportation network modeling.

The methodology framework has two advantages over the existing assignmentbased dynamic O-D matrix estimation models. First, it combines an initial O-D estimation model into the estimation process to provide a high confidence level of initial input for the dynamic O-D estimation model, which has the potential to improve the final estimation results and reduce the associated computation time.

Second, the proposed methodology framework can automatically convert traffic volume deviation to traffic density deviation in the objective function under congested traffic conditions. Traffic density is a better indicator for traffic demand than traffic volume under congested traffic condition, thus the conversion can contribute to improving the estimation performance. 
The proposed method indicates a better performance than a typical assignmentbased estimation model (Zhou et al., 2003) in several case studies. In the case study for I95 in Miami-Dade County, Florida, the proposed method produces a good result in seven iterations, with a root mean square percentage error (RMSPE) of 0.010 for traffic volume and a RMSPE of 0.283 for speed. In contrast, Zhou's model requires 50 iterations to obtain a RMSPE of 0.023 for volume and a RMSPE of 0.285 for speed. In the case study for Jacksonville, Florida, the proposed method reaches a convergent solution in 16 iterations with a RMSPE of 0.045 for volume and a RMSPE of 0.110 for speed, while Zhou's model needs 10 iterations to obtain the best solution, with a RMSPE of 0.168 for volume and a RMSPE of 0.179 for speed.

The successful application of the proposed methodology framework to real road networks demonstrates its ability to provide results both with satisfactory accuracy and within a reasonable time, thus establishing its potential usefulness to support dynamic traffic assignment modeling, ITS systems, and other strategies. 


\section{TABLE OF CONTENTS}

CHAPTER $\quad$ PAGE

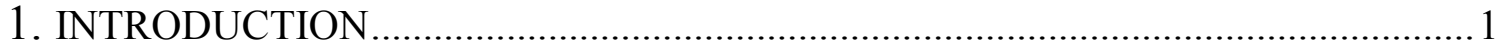

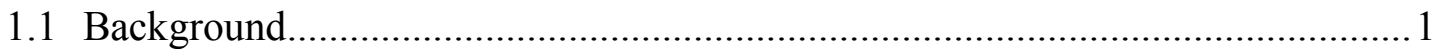

1.2 Problem Statement.......................................................................................

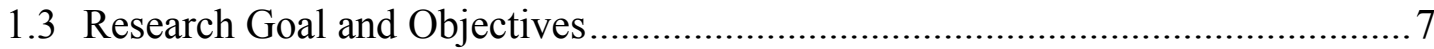

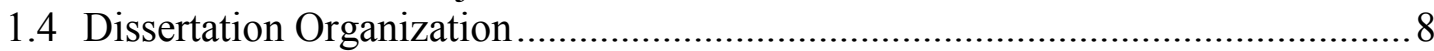

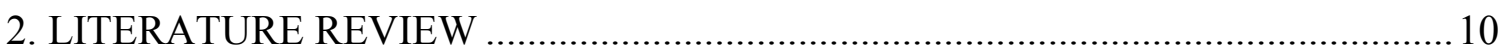

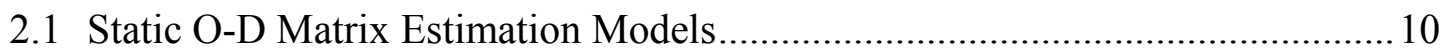

2.2 Dynamic O-D Matrix Estimation Models ……………………………............. 15

2.2.1 Assignment-Based Estimation Models ................................................. 15

2.2.2 Non-Assignment-based Estimation Models ………………………........ 35

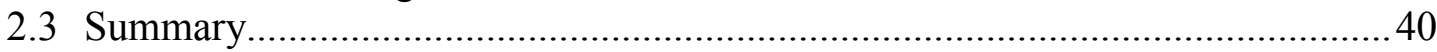

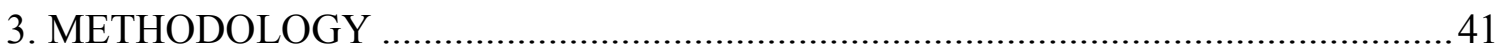

3.1 Methodology Framework …………………………..................................... 41

3.2 DTA Traffic Flow Model Calibration (TFMC) Model .........................................42

3.3 Initial O-D Matrix Estimation (IODE) Model.....................................................4

3.3.1 Initial O-D Matrix Preparation............................................................4 46

3.3.2 Static Initial O-D Matrix Estimation (SIODE) …………........................48

3.3.3 Dynamic Initial O-D Matrix Estimation (DIODE) ………………......50

3.4 Dynamic O-D Matrix Estimation (DODE) ………….....................................56

3.5 Traffic Flow Model Fine-Tuning (TFMFT) Model..............................................64

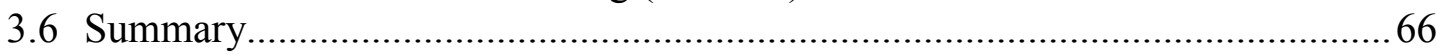

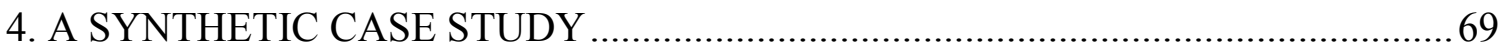

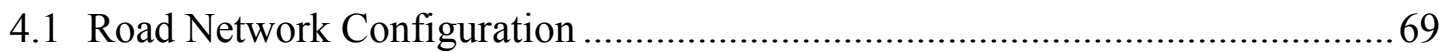

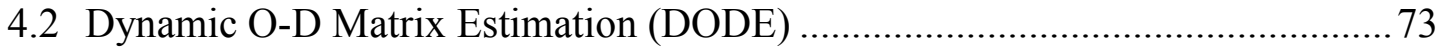

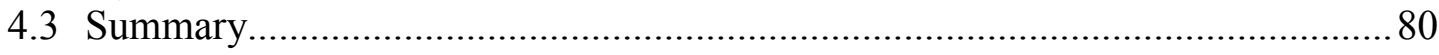

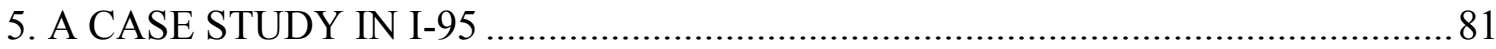

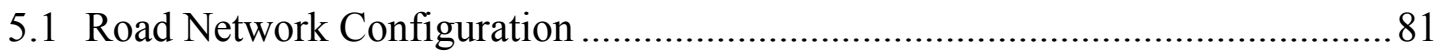

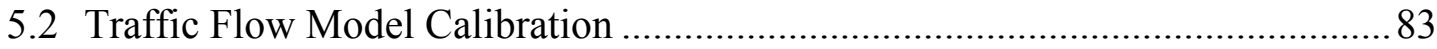

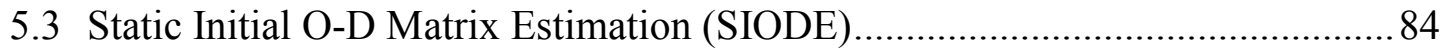

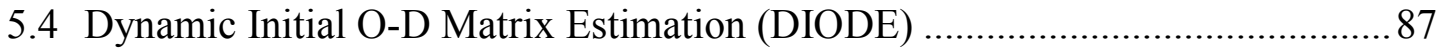

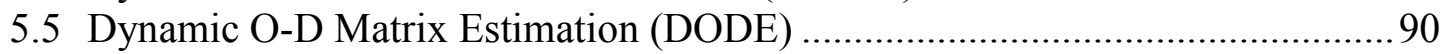

5.6 Traffic Flow Model Fine Tuning (TFMFT) ………………………………........95

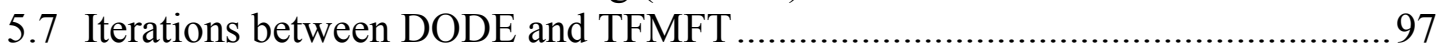

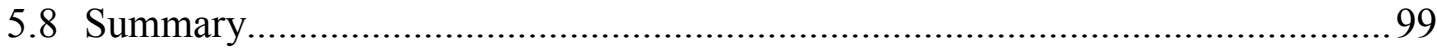

6. A CASE STUDY OF JACKSONVILLE …………............................................... 101

6.1 Subarea Road Network and Initial Traffic Demand Preparation ....................... 101

6.2 Traffic Data Preparation and Traffic Flow Model Calibration ............................ 103 


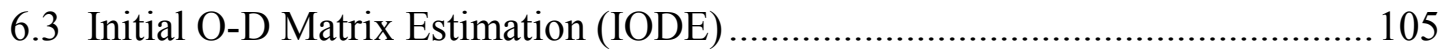

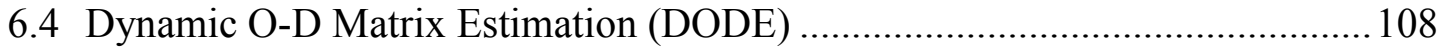

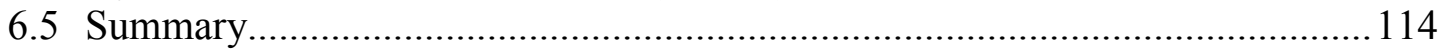

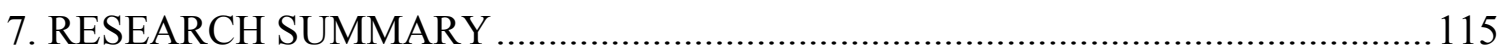

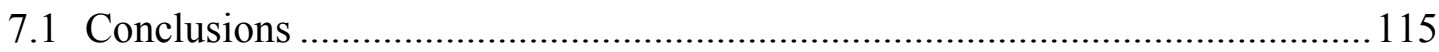

7.2 Limitations and Future Work ................................................................... 117

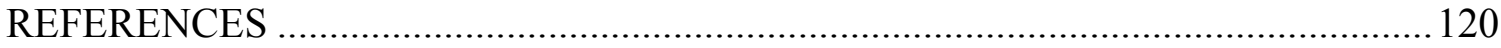

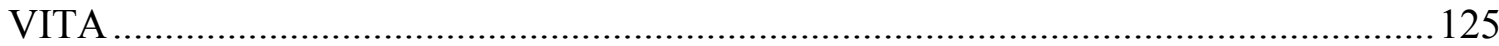




\section{LIST OF TABLES}

TABLE

PAGE

Table 4.1 Real and initial O-D demand. ........................................................... 71

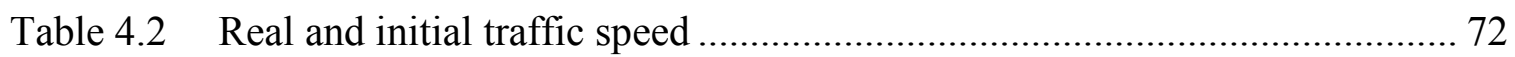

Table 4.3 Real and initial traffic volume ........................................................... 73

Table 4.4 Real and estimated O-D demand. ........................................................ 75

Table 4.5 Real and estimated traffic speed. ........................................................ 75

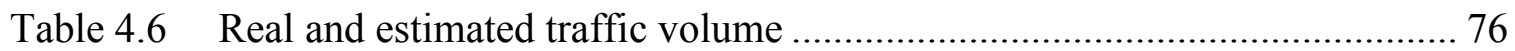

Table 4.7 The comparison of the estimation results

before-and-after the proposed terms are used.................................................... 78

Table 4.8 The comparison of the estimation result based on the proposed DODE and Zhou's estimation model. ................................................. 79

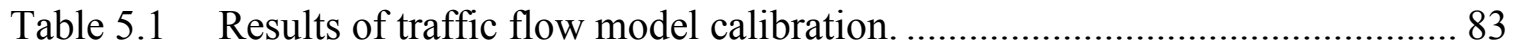

Table 5.2 Comparison between static assignment traffic volumes

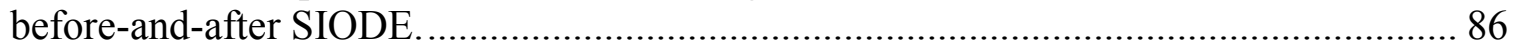

Table 5.3 Comparison of traffic volume RMSPE before-and-after DIODE. .............. 90

Table 5.4 Comparison of assignment traffic volume before-and-after DODE........... 92

Table 5.5 Comparison of traffic speed before and after DODE. .............................. 93

Table 5.6 Comparison of traffic volume and speed based on

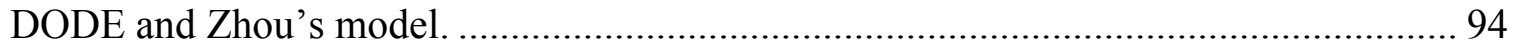

Table 5.7 Traffic volume and speed comparison after TFMFT ................................ 96

Table 5.8 Results of traffic flow model calibration. ........................................... 97

Table 5.9 Final assignment traffic volume and traffic speed comparison. .................. 98

Table 6.1 Detector and road link.................................................................. 103

Table 6.2 Traffic flow model parameters.......................................................... 105 


\section{LIST OF FIGURES}

FIGURE

PAGE

Figure 1.1 The relationship between link traffic volume

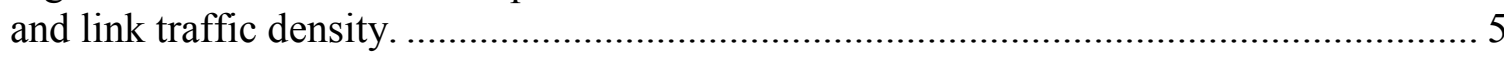

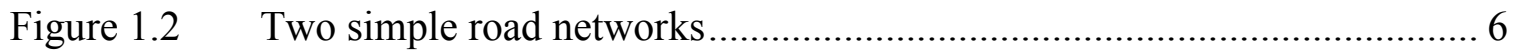

Figure 2.1 A typical freeway corridor............................................................. 35

Figure 3.1 The methodology framework of the study....................................... 42

Figure 3.2 Type 1 traffic flow model (Source: Mahmassani et al., 2005). ............... 44

Figure 3.3 Type 2 traffic flow model (Source: Mahmassani et al., 2005). ............... 44

Figure 3.4 Calibration of Type 2 traffic flow model.......................................... 45

Figure 3.5 Procedure for static initial O-D matrix estimation............................... 50

Figure 3.6 The systematic link volume deviation. ........................................... 51

Figure 3.7 The distribution of volume deviation after

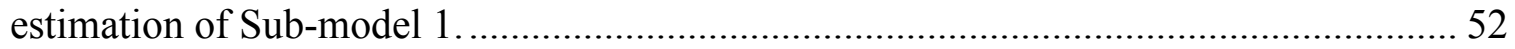

Figure 3.8 The distribution of volume deviation after

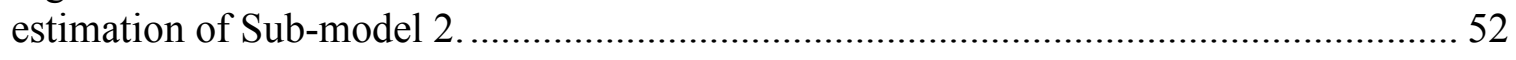

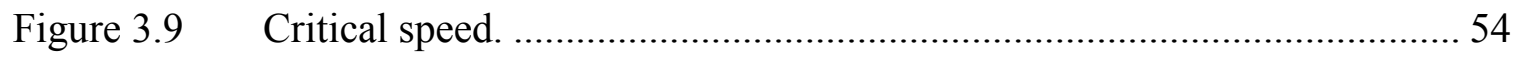

Figure 3.10 The procedure to perform DIODE ................................................. 56

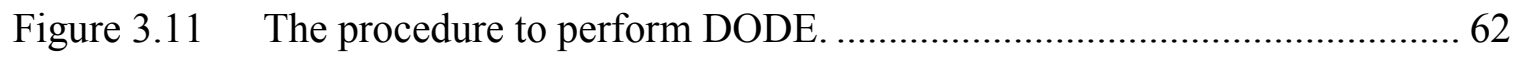

Figure 4.1 A hypothetical freeway road network............................................. 70

Figure $4.2 \quad$ Assumed traffic flow model.......................................................... 71

Figure 4.3 Real and initial simulated traffic speed from the detector...................... 72

Figure 4.4 Real and initial simulated traffic volume from the detector. .................. 72

Figure 4.5 Performance of the proposed dynamic O-D matrix

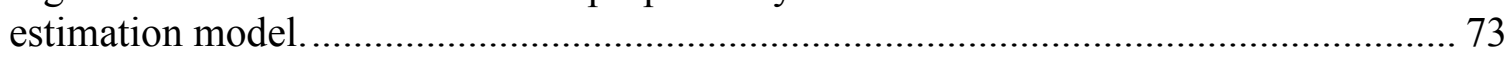

Figure 4.6 The comparison among real demand, initial demand and the estimated demand after the first iteration. 
Figure 4.7 The comparison between real speed and estimated speed..................... 75

Figure 4.8 The comparison between real volume and estimated volume. ................ 76

Figure 4.9 The comparison among the model performance

before-and-after the proposed terms are employed. ............................................... 77

Figure 4.10 Comparison of performance between the proposed

DODE model and the Zhou's model. ....................................................................... 79

Figure 5.1 Road network structure and detector locations.................................. 82

Figure 5.2 A typical traffic flow model calibration.......................................... 84

Figure 5.3 Flowchart of static initial O-D matrix estimation (SIODE).................... 85

Figure 5.4 Comparison of assignment volume RMSPE

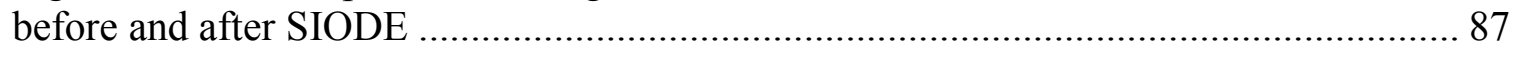

Figure 5.5 Performance of DIODE model 1. ................................................... 88

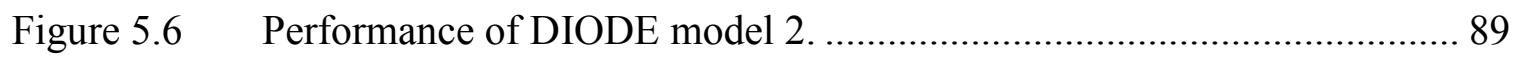

Figure 5.7 Comparison of RMSPE for assignment volume

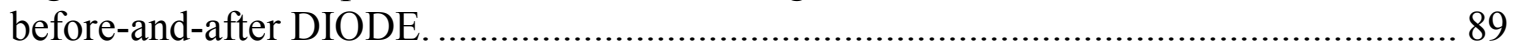

Figure 5.8 Performance of the DODE model..................................................... 91

Figure 5.9 Comparison of RMSPE for assignment volume

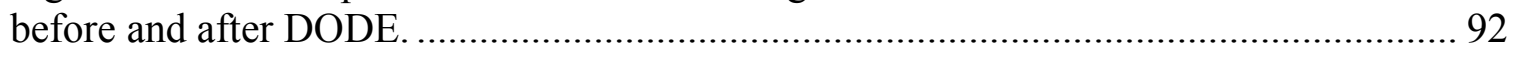

Figure 5.10 Performance of Zhou's dynamic O-D matrix estimation model. ............. 94

Figure 5.11 Performance of the successive SPSA algorithm for TFMFT. ................. 96

Figure 5.12 Observed volume versus final assignment volume............................. 99

Figure 5.13 Observed speed versus final assignment speed. .................................. 99

Figure 6.1 Subarea road network in Jacksonville, FL....................................... 102

Figure 6.2 The location of detectors in the subarea road network. ......................... 104

Figure 6.3 Comparison between assignment volume

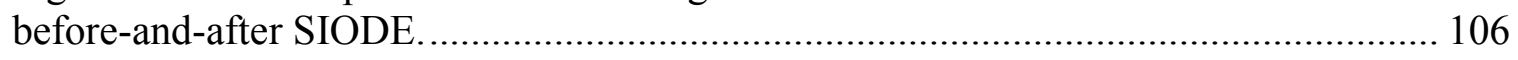

Figure 6.4 Performance of DIODE Sub-model 1............................................ 107 
Figure 6.5 Performance of DIODE Sub-model 2 ……...................................... 107

Figure 6.6 Comparison between assignment volume

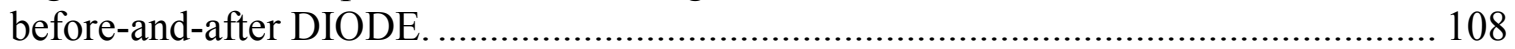

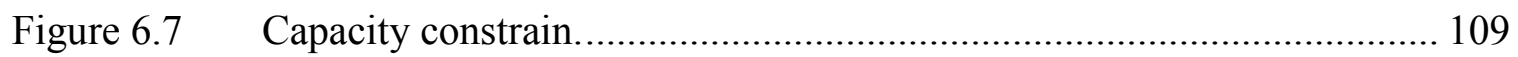

Figure 6.8 Performance of dynamic O-D matrix estimation (DODE).................... 110

Figure 6.9 Comparison between assignment volume before-and-after DODE........ 110

Figure 6.10 Assignment volumes versus observed volumes before SIODE.............. 111

Figure 6.11 Assignment volumes versus observed volumes after DODE. ................ 111

Figure 6.12 Comparison between assignment speedbefore-and-after applying the proposed methodology framework. ……………………......................... 112

Figure 6.13 Performance of Zhou's dynamic O-D matrix estimation model. ........... 113 


\section{CHAPTER 1}

\section{INTRODUCTION}

\subsection{Background}

In urban areas, traffic congestion is becoming increasingly frustrating for commuters and businesses. Despite the billions of dollars invested in transportation systems each year in America, traffic congestion continues to worsen. Schrank and Lomax (2009) estimated that, in 2007, congestion caused urban Americans to travel 4.2 billion hours more and to purchase an extra 2.8 billion gallons of fuel for a congestion cost of $\$ 87.2$ billion in 439 American urban areas. In addition to wasting fuel and money, congestion also adds to business costs, increases pollution, and contributes to various environmental problems.

Traffic congestion happens when traffic demand overwhelms the capacity of a transportation system. Solutions to the congestion problem usually focus on two principal aspects: increasing the physical capacity of the network system and reducing the travel demand. Given the limited availability of land and the negative impacts of construction on the environment, many local governments and researchers have focused on increasing the efficiency of transportation systems instead of continuing to increase road network capacity. For example, through Intelligent Transportation Systems (ITS) strategies the efficiency of transportation systems can be increased by using advanced information and telecommunication technologies. Two basic components of ITS are Advanced Traveler Information Systems (ATIS) and Advanced Traffic Management Systems (ATMS). ATIS changes travel demand patterns by providing real-time traffic information to commuters to help them better plan their trips through bypassing congested routes, choosing different departure times, or using different travel modes. ATMS uses a variety of detectors, 
cameras, and communication systems to monitor, manage, and control traffic, based on information collected from different sources.

A dynamic O-D matrix contains time dependent traffic demand information for a road network. Given a dynamic O-D matrix, it is possible to estimate and predict time dependent traffic flow in a road network. Thus, an accurate dynamic O-D matrix can act as a critical input to ITS to implement short-term traffic controls, real-time route guidance, and so on. In addition, the dynamic O-D matrix is important for the success of dynamic traffic assignment applications.

Due to the importance of dynamic O-D matrices over the past two decades, much effort has been devoted to developing effective and efficient methods to estimate dynamic O-D matrices. Existing dynamic O-D matrix estimation models can be classified into assignment-based and non-assignment-based. Assignment-based estimation models employ a dynamic traffic assignment (DTA) simulator to determine the relationship between traffic demand and traffic measurement data. They then use an optimization method to estimate a dynamic O-D matrix by minimizing the difference between the observed traffic measurements and the simulated ones. Representative works include those by Ashok and Ben-Akiva (1993, 2000, and 2002) and Zhou et al. (2006 and 2007). The advantages of assignment-based dynamic O-D matrix estimation models are, (1) they have a simple model structure; (2) they can be applied to large road networks; and (3) they can easily combine available traffic information, such as automatic vehicle identification (AVI) data, into the estimation function to improve accuracy. In contrast, the drawbacks of assignment-based O-D matrix estimation models are, (1) estimation results depend heavily on an initial O-D matrix, so the initial O-D matrix must first be 
accurate to get a good estimation; and (2) the DTA simulator employed needs to be accurately calibrated in order to correctly imitate travelers' behavior and traffic conditions in the real world.

Non-assignment-based models do not rely on a DTA simulator. Instead, they establish relationships between dynamic traffic demand and traffic measurements based on the traffic conservation relationship between trips at entries, exits, and mainstreams of a road network. Studies by Cremer Keller (1981, 1984, and 1987), Bell (1991a and 1991b), Chang and Wu (1994), and Lin (2006) fall into this category. Although nonassignment-based dynamic O-D matrix estimation models are independent of a DTA simulator, they still have problems in three aspects: (1) the traffic conservation equations cannot describe complicated traffic situations such as queuing and signal delay; (2) the traffic conservation models are too cumbersome to apply accurately to a large road network; (3) extra traffic measurement data, such as AVI data, are not easy to integrate into the model. Currently, the main applications of non-assignment-based models are for freeway segments.

Despite the progress made by researchers in the past 20 years, developing an improved model for dynamic O-D matrix estimations based on available traffic measurements remains a challenge.

\subsection{Problem Statement}

Assignment based dynamic O-D matrix estimation models have attracted an increasing amount of attention because they do not require the construction of complicated traffic conservation equations. This dissertation focuses on improving assignment-based dynamic O-D matrix estimation models. 
A general O-D matrix estimation is the inverse process of a traffic assignment, with the observed traffic measurement as input and the traffic demand as output. The criterion used in the estimation addresses if the estimated demand can reproduce observed traffic conditions. Existing assignment-based dynamic O-D matrix estimation models have two types of problems: (1) they implicitly contain a problematical assumption, namely the proportional assignment assumption defined by Bell and Lida (1997), meaning a doubling in demand causes a doubling in traffic volume; and (2) they ignore the initial O-D matrix estimation.

With the implicit proportional assumption, the existing assignment-based dynamic O-D matrix estimation models try to minimize the deviation between assignment and observed traffic volume, based on a fixed linear relationship between the O-D demand and assignment traffic volume. This relationship is often denoted as the dynamic traffic mapping matrix.

The proportional assumption ignores the real relationship between traffic demand and traffic volume, as illustrated by Figure 1.1, based on the modified Greenshields model (Mahmasani et al., 2005). Under the proportional assumption, traffic volume is assumed to have the positive proportional relationship with traffic demand. However, Figure 1.1 illustrates that the positive proportional relationship between traffic volume and traffic demand only holds true in Region A, indicated by the solid line, where traffic volume is below road capacity. In Region B of Figure 1.1, upon reaching the link's capacity, the relationship between traffic volume and demand becomes negative. Clearly, in Region B, the assumption is totally inapplicable, as increasing traffic demand only causes traffic volume to drop. 


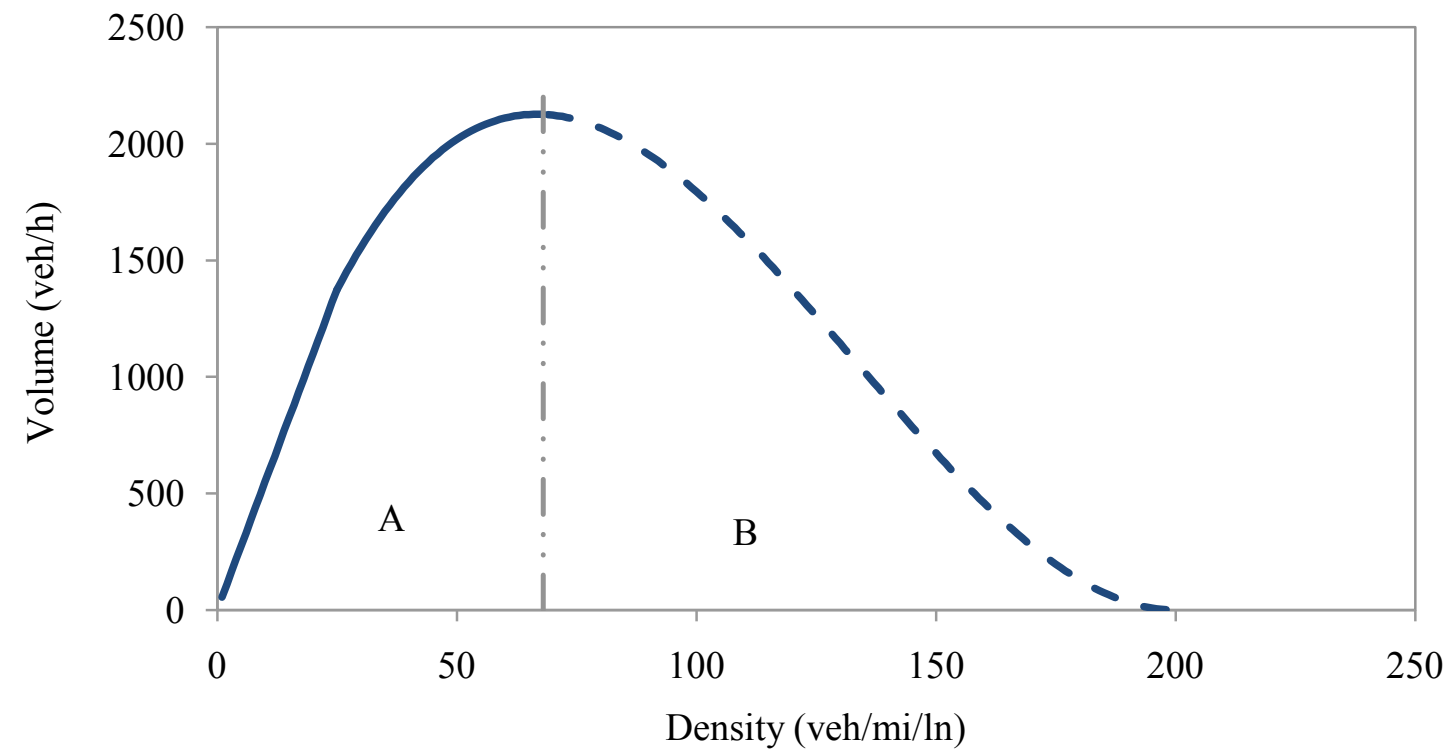

Figure 1.1 The relationship between link traffic volume and link traffic density.

The proportional assignment assumption cannot be entirely abandoned for its weakness, as analyzed above. The point is that the link traffic condition needs to be identified before applying the assumption. The assumption can be used for those links with traffic conditions that fall in Region A, as shown in Figure 1.1. In Region B, some necessary modifications to the assumption are needed.

The second problem of the existing dynamic O-D matrix model is the ignorance of the initial O-D matrix estimation. An inaccurate initial O-D matrix can cause problems in two aspects:

1. First, if an initial O-D matrix is much larger than the real one, then the traffic conditions in many road links will fall in Region B of Figure 1.1. Under this condition, it will be much less likely for the existing estimation model to yield an accurate result because the proportional assignment assumption cannot hold true in Region B. 
2. Secondly, the final estimation result relies heavily on the initial O-D matrix because of the limited information from an insufficient number of traffic detectors in a road network. Known as an under-specification problem, Zhou and Mahmassani (2006) define it as when the number of estimated, unknown O-D demand is higher than the available constraints. The under-specification problem can be seen in the example shown by the hypothetical road networks of Figure 1.2.

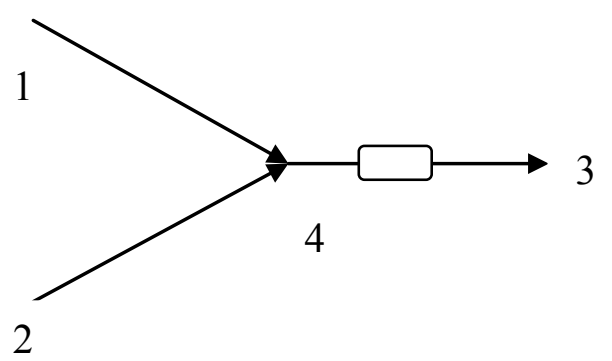

(a)

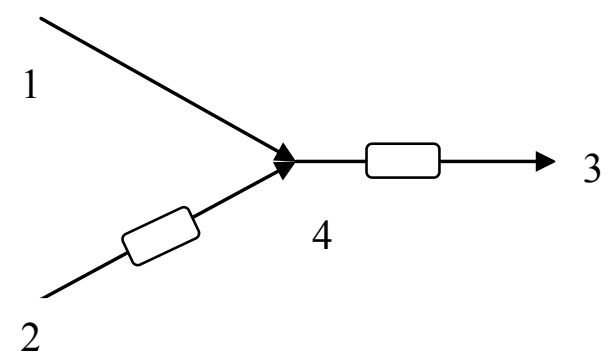

(b)

Figure 1.2 Two simple road networks

In Figure 1.2 (a), there are two O-D pairs $(1 \rightarrow 3$ and $2 \rightarrow 3)$ with only one detector installed in link 4-3, thus the O-D matrix estimation for the network in Figure 1.1 (a) has the under-specification problem. In order to see the impact of the under-specification problem, two scenarios can be assumed in the hypothetical network: (1) the detector observed volume is 100 , and initial trips for $1 \rightarrow 3$ and $2 \rightarrow 3$ are assumed 10 and 10 ; and (2)

the detector observed volume is 100 , and initial trips for $1 \rightarrow 3$ and $2 \rightarrow 3$ are assumed as 10 and 0 . Based on the proportional assumption, the estimation result for scenario (1) is 50 for $1 \rightarrow 3$ and $2 \rightarrow 3$, and the result for scenario (2) is 100 for $1 \rightarrow 3$ and 0 for $2 \rightarrow 3$. Differences in the initial O-D demand can result in different estimations with under- 
specification problem. The O-D matrix estimation for the road network in Figure 1.2 (b)

does not have the under-specification problem because there are as many detectors as unknown demands.

An accurate initial O-D matrix can provide not only the approximate demand information for an individual O-D pair but also useful information on traffic demand temporal distribution pattern. Suggested by Lin (2006), the accuracy of an initial O-D matrix is important for the estimation result. However, current assignment-based dynamic O-D matrix estimation studies ignore the estimation of an initial O-D matrix.

\subsection{Research Goal and Objectives}

Designed to develop an assignment-based dynamic O-D matrix estimation framework both efficient in computation and accurate in estimation, this study has the following main objectives:

1. Develop an initial O-D matrix estimation model to provide an improved initial OD matrix for the proposed dynamic O-D matrix estimation model and thereby increase the accuracy of the final estimation results.

2. Develop a dynamic O-D matrix estimation model with efficient and accurate estimation performance under congested traffic conditions. The model is designed to detect links with heavy simulated traffic congestion in Region B of Figure 1.1 (where the proportional assumption cannot hold), and then modify the corresponding element in the dynamic mapping matrix to improve the performance of the dynamic O-D matrix estimation under congested traffic conditions. 


\subsection{Dissertation Organization}

This dissertation consists of seven chapters. Chapter 1 introduces the background of the research, describes the major problems, and sets forth the project's goals and objectives.

Chapter 2 presents an extensive literature review covering classic static O-D matrix estimation models, assignment-based dynamic O-D matrix estimation models, and non-assignment-based dynamic O-D matrix estimation models. The purpose of the review is to understand the current studies in order to analyze the existing problems in those models.

Chapter 3 proposes a complete methodology framework. In response to the problems stated in Chapter 1, this chapter puts forward a series of models: a traffic flow model calibration (TFMC) model, an initial O-D matrix estimation (IODE) model, a dynamic O-D matrix estimation (DODE) model, and a traffic flow model fine-tuning (TFMFT) model.

Chapter 4 uses a hypothetical road network to test the dynamic O-D matrix estimation (DODE) model in the proposed methodology framework. In this case study, with the simulation data, each term in the estimation model is analyzed. Finally, a comparison of the DODE model's performance with that of the existing one demonstrates the advantage of the proposed model.

Chapter 5 applies the proposed methodology to a road segment of I-95. The case study is designed to test the feasibility and efficiency of the proposed methodology for a middle-sized road network.

Chapter 6 applies the proposed methodology to a regional road network from Jacksonville to test the feasibility and efficiency of the proposed methodology for a large- 
sized road network.

Chapter 7 summarizes the major research results in each chapter, draws conclusions, and recommends issues for future research. 


\section{CHAPTER 2}

\section{LITERATURE REVIEW}

This chapter reviews the literature on O-D matrix estimation models, including static and dynamic approaches. Static O-D matrix estimation models are the foundation of dynamic O-D matrix estimation approaches. There are two types of dynamic O-D matrix estimation models, namely assignment-based and non-assignment-based estimation models, and these are reviewed separately.

\subsection{Static O-D Matrix Estimation Models}

Developed mainly for the purpose of transportation planning, static O-D matrix estimation models can be categorized into two groups, namely entropy maximizationbased (EM) and econometrics based.

The EM approach means applying the concept of entropy to quantitative methods to forecast spatial interaction (Wilson, 1967). The entropy of an O-D matrix is the number of different permutations of trips. The hypothesis is that an O-D matrix that maximizes the entropy subject to the constraints of link traffic measurements would be the most likely O-D matrix.

Van Zuylen and Willumsen (1980) developed an EM-based static O-D matrix estimation model. In their study, O-D matrix estimation problems are formulated as Equations 2.1 and 2.2.

$$
\hat{T}_{i j}=\arg \max _{T_{i j}}\left[-\sum_{i j}\left(T_{i j} \ln T_{i j}-T_{i j}\right)\right]
$$

subject to

$$
V_{a}=\sum_{i j} T_{i j} P_{i j}^{a}
$$


where $T_{i j}$ is the O-D trips from $i$ to $j$ that are to be estimated; $\hat{T}_{i j}$ is the best estimation of $T_{i j} ; P_{i j}^{a}$ is the proportion of O-D demand from $i$ to $j$ passing link $a$; and $V_{a}$ is the observed traffic volume on link $a$.

The advantages of the EM approaches are, (1) their full utilization of observed data; (2) their ability to easily incorporate prior estimations of O-D matrices; (3) their ability to produce estimation when knowledge of travel behavior is lacking; and (4) their potential application for equilibrium assignment. However, EM approaches assume that link flows are measured without error, which conflicts with real situations; furthermore, the assumption of EM approaches may not be consistent with the traveler's route choice behaviors in the real world.

Econometrics based approaches aim to build statistical models between O-D matrices and measured traffic counts. Econometrics approaches can be categorized into three groups: maximum likelihood-based (ML) models, Bayesian inference-based (BI) models, and generalized least square-based (GLS) models.

Spiess (1987) presented a ML-based O-D matrix estimation model from the observed traffic volumes of several links and a sample O-D matrix. The assumption is that the elements of a sample O-D matrix $t_{i}$ follow an independent Poisson distribution with mean $\rho_{i} T_{i}$. The coefficient $\rho_{i}$ represents the sampling rate factor for the O-D pair $T_{i}$ that is to be estimated. The proposed estimation model is presented as Equations 2.3 and 2.4, below:

$$
f\left(T_{i}\right)=\operatorname{Min} \sum_{i \in O}\left(\rho_{i} T_{i}-t_{i} \ln T_{i}\right)
$$

subject to 


$$
\sum_{i \in O} n_{i a} T_{i}=V_{a}, a \in I
$$

where the coefficient $n_{i a}$ corresponds to the proportion of trips for O-D pair $i$ that uses link $a ; V_{a}$ denotes the observed volume of link $a$; $I$ stands for the set of links with observed traffic volumes; and $O$ represents the total number of O-D pairs.

Maher (1983) and Cascetta and Nguyen (1988) proposed BI-based O-D matrix estimation models. There are three advantages to BI-based models (Maher, 1983): (1) they are flexible when considering the confidence level of an a priori O-D matrix and observed traffic volumes; (2) they can successively update O-D matrices by observed link volumes; and (3) they can produce confident intervals for the estimation results. In a typical BI model, the a priori information on trip demand $\mathbf{T}$ can be formulated as the $a$ priori probability function $g(\mathbf{T})$. Traffic volumes are considered an additional source of information about $\mathbf{T}$, with given probability $L(\mathbf{V} \mid \mathbf{T})$, and BI methods allow the combination of these two sources of information to provide the a posteriori probability function $f(\mathbf{T} \mid \mathbf{V})$ :

$$
f(\mathbf{T} \mid \mathbf{V}) \propto L(\mathbf{V} \mid \mathbf{T}) \cdot g(\mathbf{T})
$$

Based on the maximization of an a posteriori distribution, $\mathbf{T}$ can be estimated as:

$$
\mathbf{T}=\arg \max \ln f(\mathbf{T} \mid \mathbf{V})=\arg \max [\ln L(\mathbf{V} \mid \mathbf{T})+\ln g(\mathbf{T})]
$$

Generally speaking, the a priori distribution function of $\mathrm{g}(\mathbf{T})$ can be assumed to be a multinomial Poisson or a multivariate normal distribution function. The traffic volume distribution function $\mathrm{L}(\mathbf{V} \mid \mathbf{T})$ can be a Poisson or a multivariate normal likelihood function. One of the disadvantages of BI estimation is that the estimation result varies with different a priori distribution functions for $\mathrm{g}(\mathbf{T})$. Usually, the historical information 
on the large dimensional vector $\mathbf{T}$ is insufficient to derive a reliable $\mathrm{g}(\mathbf{T})$, which can render the estimation result erroneous.

Cascetta (1984) proposed a GLS-based O-D matrix estimation model. The model combines O-D demands with traffic volume through an assignment model. O-D demands are modeled as Equation 2.7, below:

$$
\hat{\mathbf{t}}=\mathbf{t}+\boldsymbol{\varepsilon}
$$

where $\hat{\mathbf{t}}$ is an estimated $\mathrm{O}-\mathrm{D}$ vector; $\mathbf{t}$ is a true $\mathrm{O}-\mathrm{D}$ vector; and $\boldsymbol{\varepsilon}$ is a random vector with mean $\mu$ and variance and covariance matrix $\mathbf{V}$.

The relationship between O-D demands and traffic volumes, also known as the assignment model, is formulated as Equation 2.8:

$$
\mathbf{f}=\hat{\mathbf{A}} \mathbf{t}+\boldsymbol{\eta}
$$

where $\mathbf{f}$ is a traffic volume vector; $\hat{\mathbf{A}}$ is an assignment matrix; and $\boldsymbol{\eta}$ is a random vector with mean $\boldsymbol{\delta}$ and variance and covariance matrix $\mathbf{W}$.

Based on Equations 2.7 and 2.8, Cascetta (1984) models the O-D matrix estimation problem as Equation 2.9:

$$
\min _{\mathbf{t}}\left[\begin{array}{c}
\hat{\mathbf{t}}-\mathbf{t} \\
\hat{\mathbf{f}}-\hat{\mathbf{A}} \mathbf{t}
\end{array}\right]\left[\begin{array}{cc}
\mathbf{V}^{-1} & \mathbf{O} \\
\mathbf{O} & \mathbf{W}^{-1}
\end{array}\right]\left[\begin{array}{c}
\hat{\mathbf{t}}-\mathbf{t} \\
\hat{\mathbf{f}}-\hat{\mathbf{A}} \mathbf{t}
\end{array}\right]
$$

In the above GLS model, if the random vector $(\varepsilon, \eta)^{\mathrm{T}}$ follows a multivariate normal distribution with a mean of 0 , the estimator is the best linear unbiased estimator (BLUE) of the O-D matrix $\mathbf{t}$, which is the same as the maximum likelihood estimator. Generally speaking, GLS models can account for measurement errors in traffic flows 
explicitly. The disadvantage of GLS models is that the results may contain bias due to error in the initial O-D matrices' values and from the assignment models.

To solve the O-D matrix estimation problem from available traffic measurements, a bi-level structure is often needed, where the upper-level problem minimizes a distance metric between measured and estimated traffic conditions, and the lower-level generates an equilibrium assignment mapping matrix to feed the upper level problem. Considering the heavy computation required by bi-level approaches, Nie et al. (2005) proposed a path flow estimation framework that integrates a decoupled path flow estimator (PFE) into a generalized least square (GLS) model as shown in Equation 2.10. The GLS model minimizes the combined errors from link volume and historical the O-D demand matrix.

$$
\min \mathrm{z}(\mathbf{f})=0.5(\mathbf{P f}-\mathbf{x}) \mathbf{T}^{-1}(\mathbf{P f}-\mathbf{x})+0.5(\mathbf{M f}-\mathbf{q}) \mathbf{S}^{-1}(\mathbf{M f}-\mathbf{q})
$$

subject to: $\mathbf{f} \geq 0$

where $\mathbf{P}$ represents an user equilibrium assignment mapping matrix, obtained from a Kshortest path ranking procedure; $\mathbf{M}$ is a matrix that converts equilibrium path flows to $\mathrm{O}$ D demands; $\mathbf{f}$ represents path flows; and $\mathbf{S}$ and $\mathbf{T}$ represent the variance-covariance matrices for target matrix $\mathbf{q}$ and traffic counts $\mathbf{x}$. The decoupled PFE model can exogenously calculate user-equilibrium optimal paths based on a K-shortest path algorithm, which simplifies the work in equilibrium assignment.

By relaxing the user equilibrium conditions, Nie and Zhang (2008) proposed another O-D estimation model that can incorporate travelers' route choice behavior. Through relaxation, efficient algorithm solutions can be developed to handle large-scale 
estimation problems. A column generation algorithm was used to solve the relaxed model. The relaxed model is formulated by Equation 2.11, as follows:

$$
\min \mathrm{z}(\mathbf{f})=0.5 w_{x}(\mathbf{P f}-\mathbf{x})^{2}+0.5 w_{q}(\mathbf{M f}-\mathbf{q})^{2}+\frac{1}{\theta} \sum_{a} \int_{0}^{P_{a} f} \tau_{a}(w) d w
$$

subject to: $\mathbf{f} \geq 0$

where $\mathbf{f}$ is a vector of path flows; $\mathbf{q}$ is a vector of historical travel demands; $\mathbf{x}$ is a vector of measured traffic counts; $\tau_{a}(w)$ is a travel cost function of link $a ; \mathbf{P}$ and $\mathbf{M}$ are corresponding path-link incidence and path-OD incidence matrices; $w_{x}>0$ and $w_{q}>0$ are relative weight factor for $\mathbf{x}$ and $\mathbf{q}$; and $\theta$ is a dispersion parameter for travel cost.

\subsection{Dynamic O-D Matrix Estimation Models}

A dynamic O-D matrix provides the critical input information for implementing and evaluating traffic management strategies. According to Lin (2006), dynamic O-D matrix estimation models can be categorized into two groups: assignment-based and nonassignment-based. In the following two subsections, these two methods are reviewed.

\subsubsection{Assignment-Based Estimation Models}

With the assistance of DTA simulators to obtain complex traffic dynamics, assignment based dynamic O-D matrix estimation models yield the best estimation by minimizing the difference between observed traffic measurements and simulated ones. Based on modeling approaches, assignment-based dynamic O-D matrix estimation models can be classified into three groups: generalized least square-based (GLS) models, variational inequality-based (VI) models, and state-space-based models. Currently, with more traffic data available, people are trying to combine traffic data from different resources into 
dynamic O-D estimation models. As such, the last subsection will introduce some studies on the dynamic O-D matrix estimation models that use combination of AVI data, called AVI data-based estimation models. The following subsections review the four dynamic O-D matrix estimation models.

\subsubsection{GLS based Estimation Models}

This group of models extends the static GLS O-D matrix estimation models by adding a temporal dimension in both O-D matrices and traffic measurement data.

Cascetta et al. (1993) transformed static GLS O-D matrix estimation models into dynamic ones. In their study, traffic volume can be formulated as shown in Equation 2.12, below:

$$
\hat{\mathbf{V}}_{l h}=\mathbf{V}_{l h}+\mathbf{W}_{l h}
$$

where $\hat{\mathbf{V}}_{l h}$ is a measured link volume vector for link $l$ in time interval $h$; $\mathbf{V}_{l h}$ is a real link volume vector for link $l$ in time interval $h$; and $\mathbf{W}_{l h}$ is a random term.

Link volume $\mathbf{V}_{l h}$ results from O-D demand passing link $l$ during time interval $h$, and the relation between O-D matrices and link volumes can be formulated as Equation 2.13, shown below:

$$
\mathbf{V}_{l h}=\sum_{t=1}^{h} \sum_{r} \mathbf{p}_{l h}^{r t} \mathbf{d}_{r t}
$$

where $\mathbf{d}_{r t}$ is a vector of a time-dependent O-D matrix, and $\mathbf{p}_{l h}^{r t}$ is the fraction of O-D matrix $\mathbf{d}_{r t}$ which contributes to the observed traffic volume of link $l . \mathbf{p}_{l h}^{r t}$ can be formulated as the product of link-path fraction and route choice probability, as shown in Equation 2.14 below: 


$$
\mathbf{P}_{l h}^{r t}=\sum_{k \in K_{r}} \boldsymbol{\alpha}_{l h}^{k t} \mathbf{q}(k, t)
$$

where $\boldsymbol{\alpha}_{l h}^{k t}$ is a link path incidence fraction, and $\mathbf{q}(k, t)$ is the probability of an O-D trip choosing path $k$ with departure time interval $t$.

In actual applications, $\hat{\mathbf{q}}(k, t)$ and $\hat{\boldsymbol{\alpha}}_{l h}^{k t}$ may be obtained through the simulation result from a DTA model, which is subject to estimation errors. Therefore, the relationship between O-D matrices and link volumes can be revised as follows:

$$
\hat{\mathbf{V}}_{l h}=\sum_{t=1}^{h} \sum_{r} \hat{\mathbf{P}}_{l h}^{r t} \mathbf{d}_{r t}+\pi_{h}
$$

where $\pi_{h}$ is a random error vector.

After redefining the dynamic relationship between the dynamic O-D matrices and link volumes, the dynamic O-D matrix estimation model can be represented as a GLS:

$$
\mathbf{d}=\arg \min _{\mathbf{s} \in \mathbf{S}}\left[\mathrm{f}_{1}(\mathbf{s}, \hat{\mathbf{d}})+\mathrm{f}_{2}(\mathbf{v}, \hat{\mathbf{v}})\right]
$$

where $\mathbf{d}$ is a dynamic $\mathrm{O}-\mathrm{D}$ demand vector; $\mathbf{s}$ is an a priori known dynamic O-D demand vector (sample O-D matrix); $\mathbf{S}$ is a feasible set of $\mathbf{s}$; and $f_{1}(\cdot)$ and $f_{2}(\cdot)$ measure the distance between observed and estimated measurements.

Sherali and Park (2001) developed a dynamic path flow estimation method based on an optimization algorithm that uses link volume data in a general road network. The proposed model aims to determine the path flows with the least cost O-D paths and the least deviation between assignment and observed link traffic volumes. The developed model can be decomposed into a restricted master programming model and a subprogramming model. The purpose of the master programming model is to find a set of path flows that minimize the combination of the total network travel cost and the 
difference between the assignment and the observed link volumes. The sub-programming model's purpose is to solve the problem of a time dependent shortest path on an expanded time-space network. The result of the sub-programming model feeds into the master programming model for the next round of estimation. The proposed methodology is conducive only for off-line processing purposes. A constrained least square model formulates the master-programming model in their study, as shown by Equations 2.17 and 2.18 below:

$$
\begin{aligned}
& \text { Minimize } \mathbf{z}(\mathbf{f})=\frac{1}{2} \sum_{h=1}^{T} \sum_{l \in L}\left[\sum_{r \in O D} \sum_{k \in K_{r}} \sum_{t \in T} P_{l h}^{k t r} f_{k t}^{r}-\hat{y}_{l h}\right]^{2}+\mu \sum_{r \in O D} \sum_{k \in K} \sum_{t=1}^{T} c_{k t}^{r} f_{k t}^{r} \\
& f_{k t}^{r} \geq 0
\end{aligned}
$$

where $r$ is the number of O-D pairs; $k$ is the number of routes connecting a given O-D pair; $l$ is the number of links with measured traffic volumes; $h$ is a specific time interval; $f_{k t}^{r}$ is the dynamic O-D route flow for O-D pair $r$ departing at interval $t$ in route $k ; \hat{y}_{l h}$ is the traffic volume on link $l$ and during interval $h ; P_{l h}^{k t r}$ is the proportion of $f_{k t}^{r}$ contributing to $\hat{y}_{l h} ; c_{k t}^{r}$ is the travel delay for vehicles entering route $k$ at interval $t$; and $\mu$ is the weight for path travel cost.

Gajewski et al. (2002) developed an integrated square error (L2E) dynamic O-D matrix estimation model instead of an existing least square (LS) model, which is vulnerable to the traffic measurement error. The authors assume that the possibility for measurement error in observed traffic volume is high, so the LS model is not ideal for dynamic O-D matrix estimation. In contrast, the L2E model is theoretically more robust 
than the LS model in defending against large measurement error because it measures the integrated squared difference between the observed and estimated traffic volume probability density functions. The L2E model for dynamic O-D matrix estimation is shown, as follows, in Equation 2.19:

$$
F_{L 2 E}=\min _{\mathbf{P}}\left\{\int_{-\infty}^{+\infty}\left[f\left(D_{t j} \mid \mathbf{O}_{t} \mathbf{P}_{j}\right)-f\left(D_{t j} \mid \mathbf{O}_{t} \mathbf{P}_{O j}\right)\right]^{2} d D_{t j}\right\}
$$

where $t$ is the number of time periods $(t=1,2 \ldots, T) ; D_{t j}$ is the observed traffic volume in destination $j$ during time interval $t ; \mathbf{O}_{t}$ is a row vector that stands for the original observed traffic volumes vector in time period $t$ for destination $j ; \mathbf{P}_{j}$ is a column vector that represents the estimated split proportions from all origins to destination $j ; \mathbf{P}_{O j}$ is a column vector that represents the real split proportions from all origins to destination $j$; and $f(. \mid$.) is a conditional probability distribution function. After certain approximations, Equation 2.19 can be converted into Equation 2.20, as shown:

$$
F_{L 2 E}=\min _{\mathbf{p}}\left\{\frac{1}{2 \sigma \sqrt{\pi}}-\frac{2}{T p} \sum_{t=1}^{T} \sum_{j=1}^{p} N\left(D_{t j}-\mathbf{O}_{t} \mathbf{P}_{j} ; \sigma^{2}\right)\right\}
$$

where $T$ is the total number of time periods; $\sigma$ is the standard deviation for the difference between observed estimated traffic volumes; $p$ represents the number of destinations; and $N\left(D_{t j}-\mathbf{O}_{t} \mathbf{P}_{j} ; \sigma^{2}\right)$ stands for a normal probability distribution function.

According to Equation 2.20, L2E models minimize the sum of probability density functions while the maximum likelihood estimation (MLE) or LS models minimize the negative product of probability density functions. The effect of data outliers on MLE or LS models is more severe than that on L2E models. Thus, L2E dynamic O-D matrix 
estimation models have the potential to obtain better results in the case of large data errors.

Tavana and Mahmassani (2001) propose a bi-level optimization method to estimate dynamic O-D matrices based on observed traffic measurement data. In their method, a least-squares estimation model is used at the upper-level and a DTA simulator solves a user-equilibrium problem at the lower-level. Equations 2.21, 2.22, and 2.23 show the proposed model, as follows:

Upper-level:

$Z=\sum_{l, h}\left[\sum_{t, i, j} p_{(l, h),(t, i, j)} \cdot d_{t, i, j}-c_{l, h}\right]^{2}$

subject to

$d_{t, i j} \geq 0$

Lower-level:

$$
p_{(l . h),(t, i, j)}=\text { assignment }\left|d_{(t, i, j)}\right| \text { from DTA }
$$

where $i$ and $j$ are, respectively, the origin and destination zones; $t$ and $h$ represent, respectively, trip departure time interval and link volume observation time interval; $l$ stands for the link number; $p_{(l . h),(t, i, j)}$ represents the link-flow proportion that is to the ratio of demand $d_{t, i, j}$, which flows onto link $l$ during observation interval $h ; d_{t, i, j}$ is the demand that initiates trips during time interval $t$ with origin $i$ and destination $j$; and $c_{l, h}$, is the observed traffic count from link $l$ in time interval $h$. Case studies show that when the network is congested, the proposed model cannot guarantee satisfactory estimation results because observed link volume data cannot provide enough information on the O-D pattern under congested traffic conditions. 
Zhou et al. (2003) improved the dynamic O-D matrix estimation method of Tavana and Mahmassani (2001) by incorporating multi-day link traffic volumes into the objective function. Composed of two terms, the objective function's first term is the deviation between observed and estimated link flows for multiple days, and the second term is the deviation between target and estimated demand for multiple days. The updated model can help analyze traffic demand patterns using multi-day data, and is formulated by Equations $2.24,2.25$ and 2.26 below:

Upper-level:

$$
\left.Z=\sum_{m}\left\{(1-w) \sum_{l, h}\left[\sum_{t, i, j} p_{(l, h),(t, i, j), m} \cdot d_{(t, i, j), m}-c_{(l, h), m}\right\}\right]^{2}+w \sum_{i, j}\left[\sum_{t} d_{(t, i, j), m}-g_{(i, j)}\right]^{2}\right\}
$$

subject to

$$
d_{(t, i, j), m \geq 0}
$$

Lower-level:

$$
p_{(l . h),(t, i, j), m}=\text { assigning } d_{(t, i, j)} \text { based on a DTA simulator }
$$

where $i$ and $j$ respectively stand for origin and destination zone number; $t$ and $h$ represent, respectively, trip departure time interval and link volume observation time interval; $l$ is the link number; $m$ denotes the day; $w$ is a weighting factor; $p_{(l . h),(t, i, j), m}$ is a link-flow proportion, which is the proportion of demand $d_{(t, i, j), m}$ that flows onto link $l$ during observation interval $h ; d_{(t, i, j), m}$ is the demand that activates trips during interval $t$ on day $m$ with origin $i$ and destination $j ; c_{(l, h), m}$ is the observed traffic count from link $l$ in time interval $h$ of day $m$; and $g_{(i, j)}$ is the target demand from $i$ to $j$. 
Recently, off-line GLS-based dynamic O-D matrix estimation methods (Kim, 2006 and Balakrishna et al., 2007) have provided an approach to jointly estimate timedependent O-D matrices and DTA supply-side parameters, without using traffic mapping matrices, by using a stochastic optimization algorithm. The typical model of this group has the following generalized least squared format:

$$
\begin{aligned}
& Z(\mathbf{X}, \boldsymbol{\beta})=\min \left\{\sum_{h=1}^{H}\left[\boldsymbol{\varepsilon}_{M h}^{\prime} \boldsymbol{\Omega}_{M h}^{-1} \boldsymbol{\varepsilon}_{M h}+\boldsymbol{\varepsilon}_{x h}^{\prime} \boldsymbol{\Omega}_{x h}^{-1} \boldsymbol{\varepsilon}_{x h}\right]+\boldsymbol{\varepsilon}_{\beta}^{\prime} \boldsymbol{\Omega}_{\beta}^{-1} \boldsymbol{\varepsilon}_{\beta}\right\} \\
& \boldsymbol{\varepsilon}_{M h}=\mathbf{M}_{h}-\mathbf{M}_{h}^{o} \\
& \boldsymbol{\varepsilon}_{x h}=\mathbf{X}_{h}-\mathbf{X}_{h}^{a} \\
& \boldsymbol{\varepsilon}_{x h}=\boldsymbol{\beta}_{h}-\boldsymbol{\beta}_{h}^{a} \\
& \mathbf{M}_{h}=f(\mathbf{X}, \boldsymbol{\beta}, \mathbf{G}) \\
& \mathrm{L}_{\mathbf{X}} \leq \mathbf{X} \leq \mathrm{U}_{\mathbf{x}} \\
& \mathrm{L}_{\boldsymbol{\beta}} \leq \boldsymbol{\beta} \leq \mathrm{U}_{\boldsymbol{\beta}}
\end{aligned}
$$

where $\mathbf{X}$ represents an O-D demand vector; $\boldsymbol{\beta}$ denotes the DTA simulator model parameters; M stands for observed traffic measurement data, such as speed and link volume; $\mathbf{X}_{h}^{a}$ represents a historical O-D demand vector; $\mathbf{G}$ denotes a road network; $\boldsymbol{\varepsilon}$ stands for the deviation vectors between observed or historical variables with the variables awaiting estimation; and $\mathbf{\Omega}$ represents the variance and covariance matrix for observed or historical variables.

Kim (2006) proposed a bi-level structure to jointly calibrate the dynamic O-D matrix and a micro-simulation model for a real road network in Texas. A genetic algorithm (GA) is employed to calibrate the simulation model parameters in the upper- 
level because of the significant complexity in the relationship between traffic measurements and subsequent model parameters.

Balakrishna et al. (2007) employed a stochastic approximation algorithm developed by Spall (1998) to simultaneously calibrate a mescoscopic DTA model in terms of supply parameters and demand parameters, based on the generally available sensor data. After test runs, the suggested algorithm received validation in both synthetic and real-world road networks.

Kattan and Abdulhai (2006) proposed an approach based on evolutionary algorithms (EA) to estimate dynamic O-D trip matrices. EA is potentially powerful as a global search and optimization tool. While the results of their study proved the use of EA to be better than the existing deterministic O-D matrix estimation method, the computational burden incurred by this approach remains to be problem.

\subsubsection{VI based Estimation Models}

Nie and Zhang (2008) modeled the dynamic O-D estimation problem as a variational inequality (VI) in consideration of travelers' response to congestion. By endogenetically determining the dynamic assignment matrix, the model avoids a bi-level solution structure. In their model, the path deviation for a time interval $t$ is formulated by Equation 2.34, as follows:

$$
\mathbf{d}=\frac{w_{x}}{i_{m}} \mathbf{P}^{T}(\mathbf{u}-\mathbf{P f})+w_{q} \mathbf{M}^{T}(\mathbf{q}-\mathbf{M f})
$$

where $\mathbf{P}$ is a dynamic path-link incidence matrix; $\mathbf{M}$ is a dynamic path-O-D incidence matrix; $\mathbf{u}$ and $\mathbf{q}$ are, respectively, vectors for observed traffic volume and historical O-D demand; $w_{x}$ and $w_{q}$ are, respectively, confidence levels for $\mathbf{u}$ and $\mathbf{q}$; and $i_{m}$ represents the number of measurement time intervals. 
At optimum, any user will experience a travel cost equivalent to the scaled dynamic deviation. The experienced travel cost would be always equal to or higher than the scaled dynamic deviation on any unused path. Mathematically, this implies the following:

$$
\begin{aligned}
& f_{r s}^{k t}\left(c_{r s}^{k t}-\theta d_{r s}^{k t}\right)=0 \\
& c_{r s}^{k t} \geq \theta d_{r s}^{k t}, \quad f_{r s}^{k t} \geq 0
\end{aligned}
$$

where, $f_{r s}^{k t}, c_{r s}^{k t}$, and $d_{r s}^{k t}$ are, respectively, path flow, path travel cost, and path deviation for the $k$ th path between O-D pair $r s$ with departure interval $t$; and $\theta$ is a dispersion factor, which reflects the weight of travelers' behavior.

The above optimal conditions can be transformed into a variational inequality (VI), which finds $\tilde{f}_{r s}^{k t}$ such that:

$$
\sum_{r, s, k, t}\left[\tilde{c}_{r s}^{k t}-\theta \tilde{d}_{r s}^{k t}\right]\left[f_{r s}^{k t}-\tilde{f}_{r s}^{k t}\right] \geq 0
$$

where $\tilde{c}_{r s}^{k t}$ and $\tilde{d}_{r s}^{k t}$ are generated based on $\tilde{f}_{r s}^{k t}$. A column generation algorithm is used to solve the VI model.

\subsubsection{State-Space-based Estimation Models}

As an approach to modeling a physical system, state-space models include a set of firstorder differential equations with input, output, and state variables. State-space models can act as a convenient way to model dynamic systems.

State-space models have been used in modeling the dynamic relationship between O-D demands and observed link volumes in consecutive time intervals (Okutani, 1987; Ashok and Ben-Akiva, 1993, 2000, and 2002; and Antoniou et al., 2007). A typical statespace dynamic O-D matrix estimation model consists of two parts, namely the transition function and the measurement function. 
Transition functions model the auto-regressive relationship among the O-D demand at time interval $h+1$ and those at previous time intervals, as Equation 2.38 below shows:

$$
\mathbf{X}_{h+1}=\sum_{p=h-q}^{h} \mathbf{f}_{h}^{p} \mathbf{X}_{p}+\mathbf{W}_{h}
$$

where $\mathbf{X}_{h+1}$ is the dynamic O-D demand origins during time interval $h+1 ; \mathbf{f}_{h}^{p}$ is the corresponding auto-regression coefficient matrices; $p$ is the time interval before $h+1 ; q$ is the number of lagged O-D demand affecting the O-D demand in time interval $h+1$; and $\mathbf{W}_{h}$ is a random error matrix.

Measurement functions model the relationship between the O-D demand and the link volume, as shown in Equation 2.39:

$$
\mathbf{Y}_{h}=\sum_{p=h-r}^{h} \mathbf{a}_{h}^{p} \mathbf{X}_{p}+\mathbf{V}_{h}
$$

where $\mathbf{Y}_{h}$ is the link flow matrix at time interval $h ; \mathbf{a}_{h}^{p}$ is a traffic assignment coefficient matrix; $r$ is the maximum number of time intervals taken to travel between any O-D pairs in the network; and $\mathbf{V}_{h}$ is a random measurement error matrix term.

Okutani (1987) applied a state-space model to estimate the dynamic O-D matrix for a small test network. In the model, O-D matrices were directly used as state variables. The results show that a larger traffic volumes data set leads to better estimation results.

Ashok and Ben-Akiva (1993), however, thought that Okutani's model only captured temporal interdependencies among O-D flows, but ignored structural information on O-D patterns. They used deviations of current O-D flows from the best historical estimated O-D flows as state variables in their study. Such model formulations 
indirectly take into account all the available a priori structural information, thus making the transformed variables follow a normal distribution. A normal distribution of model variables is useful in allowing the available algorithms to effectively solve the state-space model. The proposed state-space models are shown as Equation 2.40 and Equation 2.41 below:

$$
\begin{aligned}
& \mathbf{X}_{h+1}-\mathbf{X}_{h+1}^{H}=\sum_{p=h-q^{\prime}}^{h} \mathbf{f}_{h}^{p}\left(\mathbf{X}_{p}-\mathbf{X}_{p}^{H}\right)+\mathbf{W}_{h} \\
& \mathbf{y}_{h}-\mathbf{y}_{h}^{H}=\sum_{p=h-p^{\prime}}^{h} \mathbf{a}_{h}^{p}\left(\mathbf{X}_{p}-\mathbf{X}_{p}^{H}\right)+\mathbf{V}_{h}
\end{aligned}
$$

where $\mathbf{X}_{h+1}$ is the O-D vector for time interval $h+1 ; \mathbf{X}_{h+1}^{H}$ is the best historical O-D vector estimation for time interval $h+1 ; \mathbf{f}_{h}^{p}$ is a autoregressive coefficient matrix that reflects the effects of previous time interval demand deviations on the current time interval demand deviation; $\mathbf{W}_{k}$ is a vector of random errors; $\boldsymbol{y}_{h}$ is the observed traffic count vector for time interval $h ; \mathbf{y}_{h}^{H}$ is the historical observed traffic count vector for time interval $h ; \mathbf{a}_{h}^{p}$ is an assignment matrix that reflects the contribution of demand vectors from previous time intervals to $\boldsymbol{y}_{h}$; and $\mathbf{V}_{h}$ is a vector of random measurement errors on traffic counts.

Ashok and Ben-Akiva (2000) proposed an alternative method to model a statespace structure by representing O-D demands as the product of origin trip and O-D split factors (the percentage of origin traffic demand to each destination), which supposedly enhances the predictive ability of the model because of the relative stability of O-D split factors. The authors combined the trip split factors into their estimation model to improve the model performance. The two transition equations and one measurement equation are shown as Equations 2.42, 2.43, and 2.44, respectively: 


$$
\begin{aligned}
& \mathbf{t}_{h+1}-\mathbf{t}_{h+1}^{H}=\sum_{p=h-q 1^{\prime}}^{h} \boldsymbol{\Phi}_{h}^{p}\left(\mathbf{t}_{p}-\mathbf{t}_{p}^{H}\right)+\mathbf{U}_{h} \\
& \boldsymbol{\Psi}_{h+1}-\boldsymbol{\Psi}_{h+1}^{H}=\sum_{p=h-q 1^{\prime}}^{h} \boldsymbol{\gamma}_{h}^{p}\left(\boldsymbol{\Psi}_{p}-\boldsymbol{\Psi}_{p}^{H}\right)+\mathbf{W}_{h} \\
& \mathbf{y}_{h}=\sum_{p=h-p^{\prime}}^{h} \mathbf{a}_{h}^{p} \mathbf{b}_{p} \mathbf{t}_{p}+\mathbf{V}_{h}
\end{aligned}
$$

where $t_{h}$ is the origin trip vector for time interval $h ; \boldsymbol{\psi}_{h}$ is the trip split factor vector for time interval $h$; superscript $H$ means that the vector is the best historical estimation; $\boldsymbol{\Phi}_{h}^{p}$ is the transition matrix for origin trip vectors; $\boldsymbol{\gamma}_{h}^{p}$ is the transition matrix for O-D split factor vectors; $\mathbf{y}_{h}$ is the observed link count vectors; $\mathbf{a}_{h}^{p}$ is the matrix mapping the O-D demand to link volume; $\mathbf{b}_{p}$ is the trip split factor matrix, which is transformed from vector $\boldsymbol{\psi}$ and has a unique mapping relationship with vector $\boldsymbol{\psi}$; and $\mathbf{U}_{h}, \mathbf{W}_{h}$, and $\mathbf{V}_{h}$ are random error matrices.

Ashok and Ben-Akiva (2002) incorporated the stochastic character of a dynamic O-D matrix estimation model by systematically modeling the dynamic O-D mapping matrix. The dynamic O-D mapping matrix was modeled in two ways. First, the randomness of mapping matrices was directly taken into account through a new measurement equation, shown in Equation 2.45 below:

$$
\hat{\mathbf{a}}_{h}^{p}=\mathbf{a}_{h}^{p}+\mathbf{u}_{h}^{p}
$$

where $\hat{\mathbf{a}}_{h}^{p}$ is the assignment matrix calculated based on available travel times and route choice fraction, according to the method proposed by Cascetta et al. (1993), and $\mathbf{u}_{h}^{p}$ is a random error vector that stands for the error of $\hat{\mathbf{a}}_{h}^{p}$ from an inaccurate measurement of travel times and route-choice fractions $\mathbf{q}_{p}$. 
Instead of directly modeling the matrix, the mapping matrix is assumed to be only determined by O-D matrix $\mathbf{T}_{h}$ and route-choice fraction $\mathbf{q}_{h}$, so the mapping matrix is indirectly modeled with Equations 2.46 and 2.47:

$$
\begin{aligned}
& \hat{\mathbf{T}}_{h}=\mathbf{T}_{h}+\boldsymbol{\lambda}_{h} \\
& \hat{\mathbf{q}}_{h}=\mathbf{q}_{h}+\boldsymbol{\Psi}_{h}
\end{aligned}
$$

where $\hat{\mathbf{T}}_{h}$ and $\hat{\mathbf{q}}_{h}$ are, respectively, the measured values of $\mathbf{T}_{h}$ and $\mathbf{q}_{h}$, and $\boldsymbol{\lambda}_{h}$ and $\boldsymbol{\psi}_{h}$ denote random error vectors. Equations 2.45, 2.46, and 2.47 can act as additional measurement equations to produce a better estimation for the state-space model in preceding Equations 2.40 and 2.41.

Zhou and Mahmassani (2007) assert that the stationary assumption of the existing state-space mode can be violated when there are structural deviations between actual and regular O-D demands. In response, they developed a structural state-space, real-time O-D matrix estimation model. In their study, the real-time O-D demand is organized into regular demand patterns, structural deviations, and random fluctuations. The true demand, $D_{(j, \tau)}$, in the following study is modeled as a linear combination of the a priori estimation, the structural deviation, and random disturbance shown in Equation 2.48. A polynomial filter on the structural deviation is then modeled to absorb the deviation and to obtain a robust prediction, as shown below:

$$
D_{(j, \tau)}=\widetilde{D}_{(j, \tau)}^{r}+\mu_{(j, \tau)}+\varepsilon_{(j, \tau)}
$$

where $\widetilde{D}_{(j, \tau)}^{r}$ denotes an a priori estimation demand matrix for O-D pair $j$ during time interval $\tau ; \mu_{(j, \tau)}$ represents a structural deviation term; and $\varepsilon_{(j, \tau)}$ is a random disturbance term following a normal distribution with zero mean. 
The structural deviation term at time $\tau+\zeta$ is then represented by an $m$ th order polynomial function, as shown in Equation 2.49:

$$
\mu_{(j, \tau+\zeta)}=b_{0}+b_{1} \zeta+b_{2} \zeta^{2}+\ldots .+b_{\mathrm{m}} \zeta^{\mathrm{m}}
$$

where $b_{p}=\mu_{(j, \tau)}^{(p)} / p$ ! is based on Taylor's expansion formulation. The transition equation manifests as Equation 2.50, below:

$$
\mu_{(j, \tau+l)}^{(p)}=\sum_{s=p}^{m} \frac{l^{(s-p)}}{(s-p) !} \mu_{(j, \tau)}^{(s)}+w_{(j, \tau)}^{(p)}
$$

where the departure time interval $\tau=k l$, and $w_{(j, \tau)}^{(p)}$ is a random error term. The measurement equation in this study is formulated as Equation 2.51:

$$
c_{(i, t)}-\sum_{\zeta=-q}^{l-1} \sum_{j=1}^{N_{\text {od }}}\left(L P_{(i, t),(j, \tau+\zeta)} \times \widetilde{D}_{(j, \tau+\zeta)}^{r}\right)=\sum_{\zeta=-q}^{l-1} \sum_{j=1}^{N_{o d}}\left(L P_{(i, t),(j, \tau+\zeta)} \times \sum_{s=0}^{m} \frac{\zeta^{s}}{s !} \mu_{(j, \tau)}^{(s)}\right)+v_{i, t}
$$

where $c_{(i, t)}$ denotes the observed volume in link $i$ during time interval $t ; L P_{(i, t),(j, \tau+\zeta)}$ represents the link proportion factor (meaning the contribution of O-D demand affects a certain link volume); and $v_{i, t .}$ is a random error term.

Antoniou et al. (2007) present a state-space model for an online calibration of dynamic O-D matrix and time-dependent parameters of DTA systems. The state variables in the study include the parameters that need calibration during time interval $h$, including O-D flows, speed-density relationship parameters, and segment capacities. Similar to the study conducted by Ashok and Ben-Akiva (1993), this study uses the deviations of unknown variables as state variables. The transition equation of this study is formulated as Equation 2.52: 


$$
\mathbf{X}_{h+1}-\mathbf{X}_{h+1}^{H}=\sum_{q=h-p}^{h} \mathbf{f}_{q}^{h+1}\left(\mathbf{X}_{q}-\mathbf{X}_{q}^{H}\right)+\boldsymbol{\eta}_{h}
$$

where $\mathbf{X}$ stands for the state vector; subscript $h+1$ or $q$ denotes the time interval; subscript $p$ signifies the order of the relationship; superscript $H$ means that the vector

comes from the historical measurement or estimation; $\mathbf{f}_{q}^{h+1}$ is the autoregressive factors matrix capturing the impact of the state vector $\mathbf{X}_{q}$ on the state vector $\mathbf{X}_{h+1}$ during interval $h+1$; and $\boldsymbol{\eta}_{h}$ is a random error matrix. The direct measurement equation is modeled below as Equation 2.53:

$$
\mathbf{X}_{h}^{a}-\mathbf{X}_{h}^{H}=\mathbf{X}_{h}-\mathbf{X}_{h}^{H}+\mathbf{W}_{h}
$$

where $\mathbf{X}_{h}{ }^{a}$ is the a priori parameter vector for time interval $h$ and $\mathbf{W}_{h}$ is a random error vector that is uncorrelated with $\boldsymbol{\eta}_{h}$. An additional measurement equation can be formulated as the following:

$$
\mathbf{M}_{h}-\mathbf{M}_{h}^{H}=S\left(\mathbf{X}_{h}\right)-\mathbf{M}_{h}^{H}+\mathbf{V}_{h}
$$

where $S$ denotes the DTA simulator; $\mathbf{V}_{h}$ is a random error vector that is uncorrelated with $\mathbf{W}_{h}$ and $\boldsymbol{\eta}_{h} ; \mathbf{M}_{h}$ is the traffic measurement vector; and superscript $H$ signifies that the vector comes from a historical measurement. Due to the non-linear term $S\left(\mathbf{X}_{h}\right)$ introduced in Equation 2.54 above, the author proposes the use of non-linear Kalman filtering algorithms to solve the state-space model.

State-space models have the potential for online applications in O-D matrix estimation and prediction, and Kalman filtering algorithms are commonly used to solve state-space models. The limitations of state-space models in practical applications lie in two aspects: (1) calibrating the coefficients of state functions requires sufficient information from historic traffic demands, which are not easy to collect in the field; and 
(2) solving a state-space model is difficult for a large road network because it is timeconsuming to compute the inverse of a large-scale matrix, as indicated by Bierlaire and Crittin (2004). Compared with state-space models, GLS models have the advantage of a lighter computational burden, as many solution algorithms can be used to exploit the sparsity of the large-scale matrix, and thus save computation time.

\subsubsection{AVI Data-based Estimation Models}

In the real world, because there are insufficient traffic measurement data, the dynamic OD matrix estimation follows an under-determined process. More traffic information needs to be collected to relieve this problem. With the development of automatic vehicle identification (AVI) technology, AVI data, including point data (such as speed) and point-to-point traffic data (such as travel time and sample O-D demand), have become useful information sources in creating a better estimation of dynamic O-D matrices.

The information extracted from AVI data is usually incorporated into dynamic OD matrix estimation models in two ways: as observed O-D demand data and as link choice proportions data.

Van der Zijpp (1997) integrated AVI data as observed O-D demand information in a state-space dynamic O-D matrix estimation model. The result from a case study shows consistently lower estimation errors with the added AVI information. The proposed state-space model consists of Equation 2.55 as the transition equation, Equation 2.56 as the measurement equation, and Equation 2.57 as an additional measurement equation based on AVI data:

$$
\begin{aligned}
& \mathbf{b}(t+1)=\mathbf{b}(t)+\mathbf{w}(t) \\
& \mathbf{y}(t)=\mathbf{H}(t) \mathbf{b}(t)+\mathbf{v}(t)
\end{aligned}
$$




$$
\mathbf{e}(t)=\mathbf{G}(t) \mathbf{b}(t)+\mathbf{z}(t)
$$

where $t$ denotes time interval; $\mathbf{b}(t)$ is an O-D split factor vector indicating the percentage of origin trips that lead to certain destination; $\mathbf{y}(t)$ is an observed link volume vector; $\mathbf{H}(t)=\boldsymbol{\tau} \times \mathbf{q}(t)$, where $\boldsymbol{\tau}$ is a path link incidence vector reflecting if a path includes a certain link; $\mathbf{q}(t)$ is the number of vehicles entering the network; $\mathbf{e}(t)$ is an observed AVI vehicle vector that includes the number of vehicles recognized at site $a$ during period $t$ and at site $b$ during period $t_{1} ; \mathbf{G}(t)=\mathbf{q}(t) \times \mathbf{p}$, where $\mathbf{p}$ stands for the contribution of the O-D demand to a certain AVI count, and can be estimated based on a nested selection; and $\mathbf{w}(t), \mathbf{v}(t)$ and $\mathbf{z}(t)$ are uncorrelated random error matrices.

Kwon and Varaiya (2005) incorporated AVI data as a sample O-D demand matrix for the estimation model and developed a moments-based estimator. The assumption in their study is that for a large number of real O-D demand $\mathbf{N}$, by the Central Limit Theorem (CLT), collected AVI O-D demand M approximately follows a multivariate normal distribution, which can be expressed as:

$$
\mathbf{M} \sim N(\mathbf{A N}, \Sigma(\mathbf{M}))
$$

where the matrix $\mathbf{A}_{j k}=p_{j k} \times \psi, p_{j k}$ is the identification rate for vehicles with an AVI transponder from $j$ to $k$; $\psi$ is the probability that a vehicle is equipped with an AVI transponder; and $\Sigma(\cdot)$ specifies variance-covariance function. Based on the method of moments estimation, the final O-D demand vector is stated as:

$$
\hat{\mathbf{N}}=\mathbf{A}^{-1} E(\mathbf{M})
$$

where, $E($ ) stands for the expectation function. 
Antoniou et al. (2004) extracted the O-D demand information from the AVI data by introducing a diagonal expansion factor matrix and combining this demand information into Ashok's state-space model (1996) to estimate the dynamic O-D matrix. Two additional measurement equations were constructed and were integrated into a statespace model to estimate the dynamic O-D matrix. The first measurement equation relates to AVI O-D demands and historical O-D demands, and is shown as:

$$
\mathbf{Y}_{h}^{\text {probe }}=\mathbf{E}_{h} \mathbf{X}_{h}+\mathbf{u}_{h}
$$

where $\mathbf{E}_{h}$ is a diagonal matrix and represents an expansion factor that accounts for the fact that probe vehicles constitute only a fraction of the total number of vehicles in the network; $\mathbf{Y}_{h}^{\text {probe }}=\mathbf{x}_{h}^{\text {probe }}-\mathbf{E}_{h} \mathbf{x}_{h}^{H}$ is the deviation of observed probe vehicle flow $\mathbf{x}_{h}^{\text {probe }}$ from the best estimation of the available expansion matrix $\mathbf{E}_{h}$ and the historical demand; $\mathbf{X}_{h}=\mathbf{x}_{h}-\mathbf{x}_{h}^{H}$ is a vector of deviations of the O-D demand flows for time interval $h$; and $\mathbf{u}_{h}$ is a vector of Gaussian, zero-mean, uncorrelated errors.

The second additional measurement equation reflects the contribution of an AVI O-D demand flow to the sub-path flow, in terms of deviations, and is formulated as Equation 2.61, shown below:

$$
\mathbf{Z}_{h}=\mathbf{G}_{h} \mathbf{X}_{h}+\boldsymbol{\eta}_{h}
$$

where $\mathbf{Z}_{h}=\mathbf{z}_{h}-\sum_{p=h-p^{\prime}}^{h} \mathbf{G}_{h}^{p} \mathbf{x}_{p}$ is a vector of deviations on sub-path flows; $\mathbf{X}_{h}=\mathbf{x}_{h}-\mathbf{x}_{h}^{H}$ is a vector of deviations on O-D demand flows for time interval $h$; $\boldsymbol{\eta}_{h}$ is a vector of Gaussian, zero-mean, uncorrelated errors; and $\mathbf{G}_{h}^{p}$ is a matrix $\left(\mathrm{n}_{\text {subpath }} \times \mathrm{n}_{\mathrm{OD}}\right)$ that matches the sub- 
path flows observed in time interval $h$ (as obtained by a combination of sequential AVI measurements of the same vehicle) to O-D flows that departed in time interval $p$.

Zhou and Mahmassani (2006) integrated the link-to-link split factor from the AVI data into a least square dynamic O-D matrix estimation objective function, which avoids the effort of estimating the identification rates and market penetration of the AVI equipped vehicles. Their dynamic O-D matrix estimation model uses the following series of equations, as shown:

$$
Z=\min \left[Z_{1}+Z_{2}+Z_{3}\right]
$$

subject to

$\hat{p}=$ assigning traffic demand based on a DTA simulator

where

$$
\begin{aligned}
& \mathrm{Z}_{1}=w_{1} \sum_{l \in L_{l}, t}\left[c_{(l, t)}-\sum_{i, j, \tau} \hat{p}_{(l, t)(i, j, \tau)} \cdot d_{(i, j, \tau)}\right]^{2} \\
& \mathrm{Z}_{2}=w_{2} \sum_{i, j}\left[g_{(l, t)}-\sum_{\tau} d_{(i, j, \tau)}\right]^{2} \\
& \mathrm{Z}_{2}=w_{3} \sum_{l \in L_{i, t}, t s \in L_{L_{l}}}\left[\frac{c^{i d}{ }_{(l, s, t)}}{c^{i d}(l, t)}-\frac{\sum_{i, j, \tau} \hat{p}_{(l, s, t)(i, j, \tau)} \cdot d_{(i, j, \tau)}}{\sum_{i, j, \tau} \hat{p}_{(l, t)(i, j, \tau)} \cdot d_{(i, j, \tau)}}\right]^{2}
\end{aligned}
$$

where $w_{1}, w_{2}$, and $w_{3}$ are the weighting factors on deviations of link counts, historical demand, and observed split fractions; $l$ is the subscript for the link with traffic volume data measurement; $i$ is the subscript for origin zone; $j$ is the subscript for destination zones; $\tau$ is the subscript for departure time intervals; $t$ is the subscript for observation time intervals; $i d$ is the superscript for AVI vehicles; $c_{(, t)}$ is the number of observed vehicles 
on link $l$ during observation interval $t ; c_{(l, t)}^{i d}$ is the corresponding term for additional AVI vehicles; $c_{(l, s, t)}$ is the number of vehicles observed on link $s$ traveling from link $l$ during observation interval $t ; c_{(l, s, t)}^{i d}$ is the corresponding term for additional AVI vehicles; $d_{(i, j, \tau)}$ is the traffic demand with destination zone $j$ originating from zone $i$ during departure interval $\tau ; \hat{p}_{(i, t)(i, j, \tau)}$ is link flow proportions that are the proportion of vehicles from origin $i$ to destination $j$ starting their trips during departure interval $\tau$ and contributing to the flow on link $l$ during observation interval $t ; \hat{p}_{(l, s, t)}(i, j, \tau)$ is link-to-link-flow proportions that are the proportion of vehicles from origin $i$ to destination $j$ starting their trips during departure interval $\tau$ and contributing to the link-to-link flow from link $l$ to link $s$ in observation intervals $t ; g_{(i, j)}$ is the static target demand for O-D pair $(i, j) ; L_{l c}$, is the set of links with observed link count data; and $L_{V i}$ is the set of links with observed AVI data.

\subsubsection{Non-Assignment-based Estimation Models}

The models in this category estimate dynamic O-D matrices based on the traffic flow conservation equations of a road network. A typical freeway corridor is shown in Figure 2.1, where detectors are installed at ramps and mainline links. The available information from detectors are time dependent on-ramp volume $q_{i}(k)$, off-ramp volume $y_{j}(k)$, and mainline volume $U_{l}(k)$.

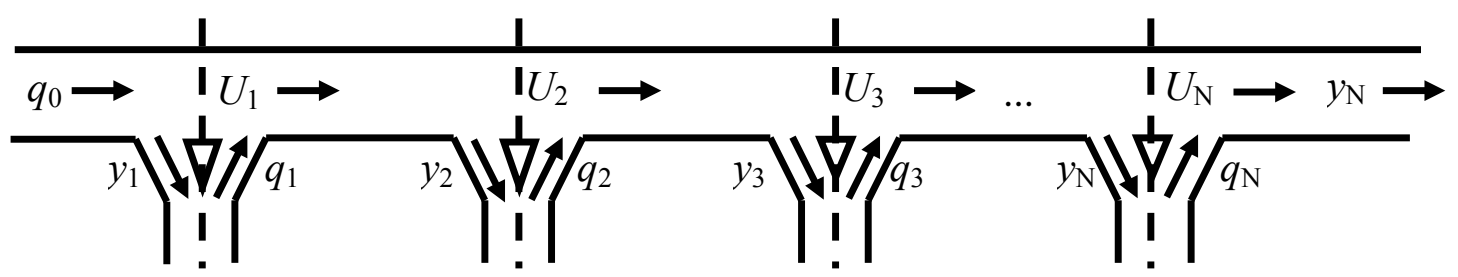

Figure 2.1 A typical freeway corridor. 
Let $b_{i j}(k)$ represent the percentage of vehicles entering the network on-ramp $i$ to off-ramp $j$ during time interval $k . b_{i j}(k)$ has its two natural constraints, as shown by Equations 2.66 and 2.67:

$$
\begin{aligned}
& 0 \leq b_{i j}(k) \leq 1,0 \leq i<j \leq N . \\
& \sum_{j=i+1}^{N} b_{i j}(k)=1, i=0,1, \ldots, N-1 .
\end{aligned}
$$

Cremer and Keller (1981) developed a dynamic O-D matrix estimation model for a small network without considering vehicle O-D travel time. Their model is formulated as follows:

$$
y_{j}(k)=\sum_{i=0}^{j-1} b_{i j}(k) q_{i}(k)
$$

subject to Equations 2.60 and 2.61

The model is useful in estimating the dynamic O-D matrix for an isolated intersection or a simple network. However, when the O-D travel time is significantly longer on a network, the model fails to generate reasonable estimation results because there are time lags between input and output flow, which are ignored by the model.

Bell (1991a) integrated a platoon dispersion factor (proposed by Roberson, 1969) into a dynamic O-D matrix estimation model for intersections. The assumption of the model is that there are geometrically distributed vehicle travel times in a road network. The assumption only holds true for short travel distance. Bell's model is shown in Equation 2.69 below:

$$
y_{j}(k)=\left(1-\alpha_{j}\right) y_{j}(k-1)+\alpha_{j} q^{T}(k) b_{i j}(k)
$$

subject to Equations 2.66 and 2.67 
where $\alpha_{j}$ is an additional smoothing parameter $\left(0 \leq \alpha_{j} \leq 1\right)$ requiring estimation. A model of Equation 2.64 can better reflect the traffic flow dynamics for an isolated intersection or small freeway network.

Bell (1991a) further extends the model to a larger freeway network by combining traffic travel time factors $b_{i j m}$ into the estimation model without considering the distribution assumption on a vehicle's travel time. $b_{i j m}$ represents the proportion of trips from entrance $i$ to destination $j$ with $m$ time intervals to travel. The model is shown in Equations 2.70, 2.71 and 2.72, as follows:

$$
\begin{aligned}
& y_{j}(k)=\sum_{m=0}^{M} \sum_{i=0}^{j-1} q_{i}(k-m) b_{i j m}(k), j=1,2, \ldots, N \\
& \sum_{j=i+1}^{N} \sum_{m=0}^{M} b_{i j m}(k)=1, i=0,1, \ldots, N-1 \\
& 0 \leq b_{i j m}(k) \leq 1,0 \leq i<j \leq N ., m=0,1, \ldots, M
\end{aligned}
$$

The above model offers a more realistic formulation, since the travel time for any O-D pair may be more than one time interval. However, the equations would involve too many unknown parameters $b_{i j m}(k)$.

Chang and $\mathrm{Wu}$ (1994) proposed a freeway O-D matrix estimation model by employing both mainline flow counts, $U_{l}(k)$, and ramp flow measurements, $q_{i}(k)$ and $y_{j}(j)$, to construct a set of dynamic equations. To further capture the relationship between O-D flow proportions and traffic counts, they proposed a set of new variables, $\theta_{i j}^{m}(k)$ and $\theta_{i l j}^{m}(k)$, to represent the fraction of $q_{i}(k-m) b_{i j}(k-m)$ trips that arrive at off-ramp $j$ and mainline $l$ during time interval $k$. The model formulations are shown as Equations 2.73, 2.74, 2.75, and 2.76 below: 


$$
\begin{aligned}
& y_{j}(k)=\sum_{m=0}^{M} \sum_{i=0}^{j-1} q_{i}(k-m) \theta_{i j}^{m}(k) b_{i j}(k-m) \quad j=1,2, \ldots, N \\
& U_{l}(k)-q_{l}(k)=\sum_{m=0}^{M} \sum_{i=0}^{l-1} \sum_{j=l+1}^{N} q_{i}(k-m) \theta_{i l}^{m}(k) b_{i j}(k-m) \quad l=1,2, \ldots, N-1
\end{aligned}
$$

subject to:

$$
\begin{aligned}
& 0 \leq \theta_{i j}^{m} \leq 1 \quad 0 \leq i<j \leq N, m=0,1,2, \ldots, M \\
& \sum_{m=0}^{M} \theta_{i j}^{m}(k+m)=0 \quad 0 \leq i<j \leq N
\end{aligned}
$$

and Equations 2.66 and 2.67

The model of Chang and $\mathrm{Wu}$ introduces a large amount of unknown parameters $b_{i j}(k)$ and $\theta_{i j}^{m}(k)$. Thus, it would be difficult for the model to solve dynamic O-D matrix estimation problems for a large road network.

In order to reduce the number of unknown variables in the model developed by Chang and $\mathrm{Wu}$, Lin (2006) assumed that the travel time for vehicles from $i$ to $j$ departing in time interval $k$ follows a normal distribution, $t_{i j} \sim N\left[u_{i j}(k), \sigma_{i j}(k)\right]$, so that it works with the travel time distribution factor $\theta_{i j}^{m}(k)=\int_{m \cdot t_{0}}^{(m+1) \cdot t_{0}} f_{i j}(x) d x$, where $f_{i j}(\mathrm{x})$ stands for the PDF function of $t_{i j}$.

Lin (2006) further assumes that travel times for vehicles from point $i$ to mainline point $l$ is $\mathrm{t}_{i l j}(k)$ and follows a normal distribution. She also assumes that the speed distribution for vehicles from $i$ to $j$ remains unchanged. Under these assumptions, travel time $t_{i j}(k)$ is proportional to travel time $\mathrm{t}_{i j}(k)$. Then the equation becomes $\mathrm{t}_{i l k}(k) \sim \mathrm{N}\left(\mathrm{r}_{t i j}\right.$, 
$\left.\mathrm{r}_{i l k} \sigma(k)\right)$, where $\mathrm{r}_{i l j}$ is the ratio of distance $d_{i l}$ to distance $d_{i j}$. The travel time distribution factor becomes $\theta_{i j j}^{m}(k)=\int_{m \cdot t_{0}}^{(m+1) t_{0}} f_{i l j}(x) d x$.

Another improvement of Lin (2006) is that the model only needs to estimate the average $\mathrm{b}_{i j}(k)$ for consecutive time intervals, namely $\bar{b}_{i j}(k)$, instead of estimating $\mathrm{b}_{i j}(k)$ for each small time interval. Finally, Lin's model is given in Equations 2.77 and 2.78 below:

$$
\begin{aligned}
& y_{j}(k)=\sum_{m=0}^{M} \sum_{i=0}^{j-1} q_{i}(k-m)\left[\int_{m \cdot t_{0}}^{(m+1) t_{0}} f_{i j}(x) d x\right] \bar{b}_{i j}(k) \quad j=1,2, \ldots, N \\
& U_{l}(k)-q_{l}(k)=\sum_{m=0}^{M} \sum_{i=0}^{l-1} \sum_{j=l+1}^{N} q_{i}(k-m)\left[\int_{m \cdot t_{0}}^{(m+1) \cdot t_{0}} f_{i j}(x) d x\right] \bar{b}_{i j}(k) \quad l=1,2, \ldots, N-1
\end{aligned}
$$

subject to Equations 2.66, 2.67, 2.75 and 2.76

where $\bar{b}_{i j}(k)=\frac{1}{M+1} \sum_{m=0}^{M} b_{i j}(k-m)$.

With Lin's improvements, the unknown variables are reduced to $\bar{b}_{i j}(k)$ and $\sigma_{i j}(k)$. Non-assignment-based dynamic O-D matrix estimation methods require the road network be "closed," meaning that all incoming and outgoing traffic must be known. Furthermore, when a network becomes too large, analytic equations will become complicated and difficult to solve. The additional traffic information, such as AVI data, is also hard to incorporate into the model. 


\subsection{Summary}

This chapter reviews the state-of-the-art, current dynamic O-D matrix estimation methods. Compared with the non-assignment-based model, the assignment-based model demonstrates preferable characters in a simpler modeling structure, in its applicability for large road networks, and in exploiting the available traffic measurement. The deficiency of the assignment-based model lies in two aspects: (1) a reliable initial O-D matrix is needed; (2) the estimation is poor in congested traffic conditions. These two problems can pose a challenge based on limited survey data. The stochastic dynamic O-D matrix estimation model (assignment-based) is a promising approach to a simpler modeling structure yielding better results, but the heavy computation burden can restrict the model when applied to a real road network. For assignment-based models, there is also a trend to integrate more available traffic data, such as AVI, into the dynamic O-D matrix estimation model to improve the estimation performance. 


\section{CHAPTER 3}

\section{METHODOLOGY}

This chapter presents the methodology proposed in this dissertation. It has two main parts: (1) an initial O-D matrix estimation method, and (2) a dynamic O-D matrix estimation model that delivers a good performance under congested traffic conditions. Section 3.1 presents the methodology framework for this study, and then describes each component of the methodology framework in detail from Sections 3.2 to 3.4.

\subsection{Methodology Framework}

The methodology framework of this study is shown in Figure 3.1. The proposed methodology framework includes the following four models:

1. Traffic flow model calibration (TFMC). A DTA simulator is used to dynamically load the traffic demand of a road network. In order to get an accurate simulation result, the traffic flow models of the DTA simulator need to be calibrated accurately based on available traffic measurement data.

2. Initial O-D matrix estimation (IODE). The model is aimed to produce an accurate initial O-D matrix in order to improve the performance of dynamic O-D matrix estimations.

3. Dynamic O-D matrix estimation (DODE). This model is designed to get an improved dynamic O-D matrix estimation under congested traffic conditions.

4. Traffic flow model fine tuning (TFMFT). This model is used to further adjust the DTA supply-side parameters based on the resulting dynamic O-D matrix from the third model. This model is iterated with the dynamic O-D matrix estimation model until a convergent result is derived. Since there is no explicit relationship 
between the DTA supply-side parameters and the traffic measurement data, only a heuristic method such as a stochastic optimization algorithm can be used to finetune the parameters. When the road network includes a large number of O-D pairs, the stochastic optimization will take too long a time to find a good solution. Thus, it is inefficient to use this model when the road network is large.

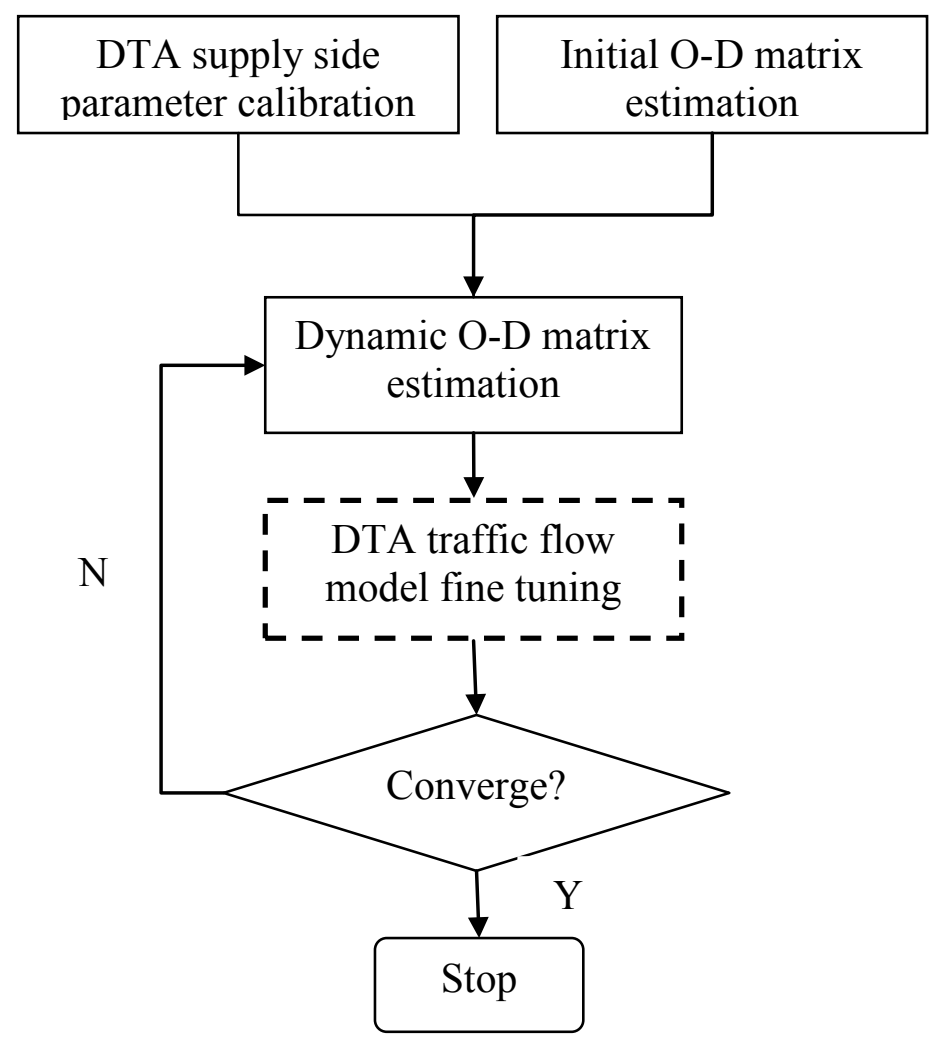

Figure 3.1 The methodology framework of the study.

The four components of the methodology framework are described separately in the following three sections.

\subsection{DTA Traffic Flow Model Calibration (TFMC) Model}

DYNASMART-P is used to perform dynamic traffic assignment (DTA). In order to better replicate real traffic situations, the traffic flow models in DYNASMART-P need to be calibrated carefully based on available traffic measurement data. 
The modified Greenshields models are used as the traffic flow model in DYNASMART-P. Two types of the modified Greenshields models are used. Type 1 is a dual-regime model for freeway links, as shown in Figure 3.2 (Mahmassani et al., 2005), and Type 2 is a single-regime model for arterial links, as shown in Figure 3.3 (Mahmassani et al., 2005). The Type 1 traffic flow model is formulated as follows, by Equations 3.1 and 3.2:

$$
\begin{array}{ll}
V_{i}=U_{f} & 0 \leq K_{i} \leq K_{b} \\
V_{i}-V_{0}=\left(V_{f}-V_{0}\right)\left[1-\frac{K_{i}}{K_{j}}\right]^{\alpha} & K_{b} \leq K_{i} \leq K_{j}
\end{array}
$$

The Type 2 traffic flow model is formulated as Equation 3.3 below:

$$
V_{i}-V_{0}=\left(V_{f}-V_{0}\right)\left[1-\frac{K_{i}}{K_{j}}\right]^{\alpha}
$$

where for road segment $i, V_{i}$ is the mean vehicle speed $(\mathrm{mi} / \mathrm{h}) ; U_{f}$ is the vehicle free speed $(\mathrm{mi} / \mathrm{h}) ; V_{0}$ is the minimum speed $(\mathrm{mi} / \mathrm{h}) ; K_{j}$ is the traffic density $(\mathrm{veh} / \mathrm{mi} / \mathrm{ln}) ; K_{b}$ is the regime's break point density ( $\mathrm{veh} / \mathrm{mi} / \mathrm{ln}) ; K_{j}$ is the jam density $(\mathrm{veh} / \mathrm{mi} / \mathrm{ln})$; and $\alpha$ is the power term used to capture the sensitivity of speed to density. 


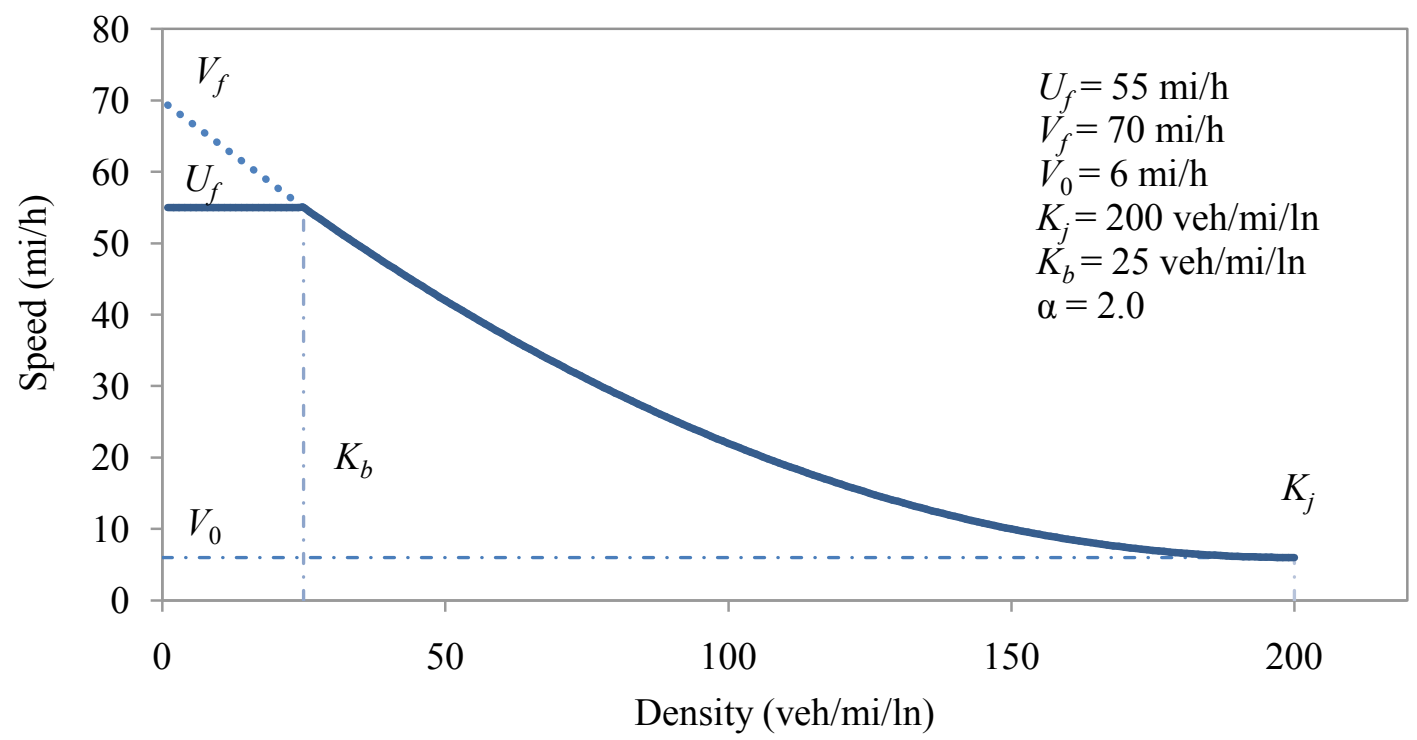

Figure 3.2 Type 1 traffic flow model (Source: Mahmassani et al., 2005).

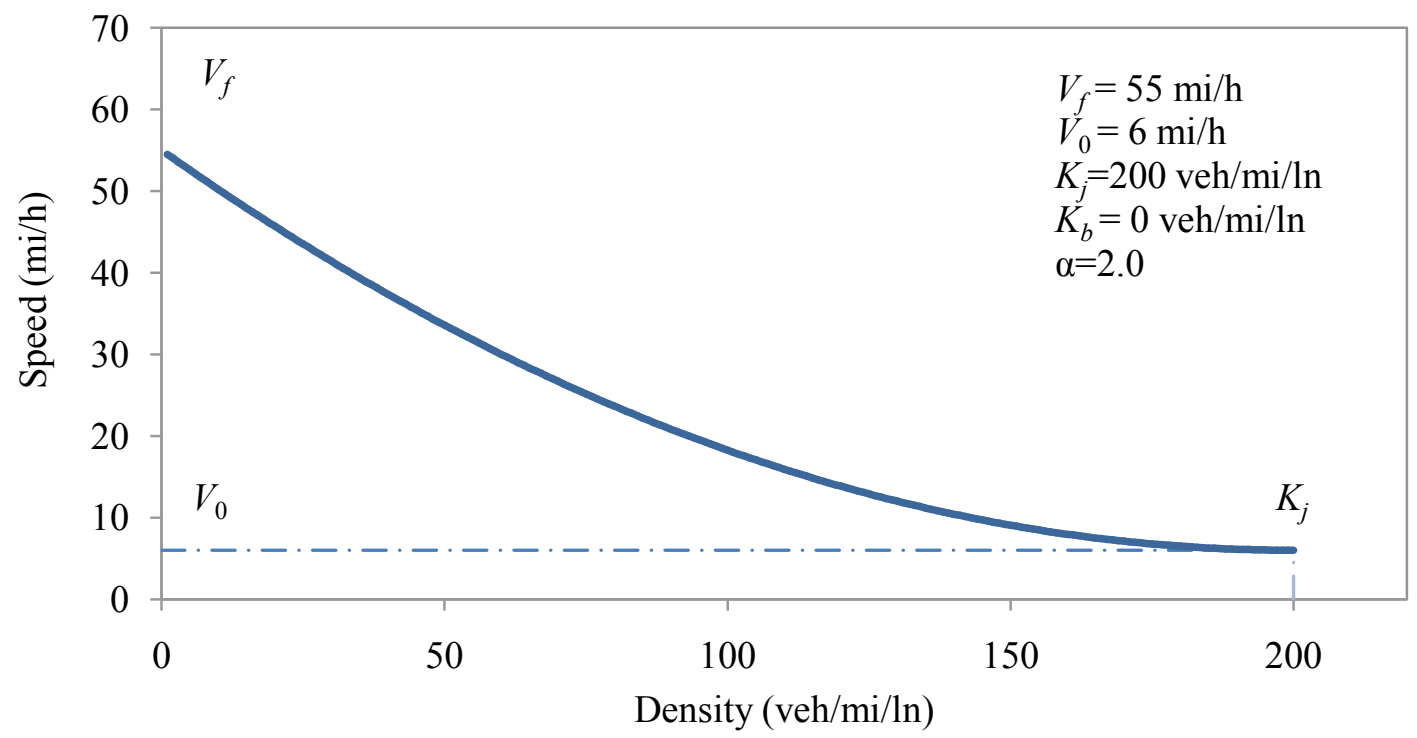

Figure 3.3 Type 2 traffic flow model (Source: Mahmassani et al., 2005).

The two-regime model is used for the freeway links where traffic speed is relatively insensitive to traffic volume in uncongested traffic conditions. The singleregime model is applied to arterial links because arterials have intersections, and the travel speed on arterials is more sensitive to increases in traffic. The parameters of the traffic flow model can be calibrated by using a linear regression technique based on 
available traffic measurement data, as illustrated in Figure 3.4, for the Type 2 traffic flow model calibration. The calibration of the Type 1 traffic flow model is similar to that of the Type 2 model.

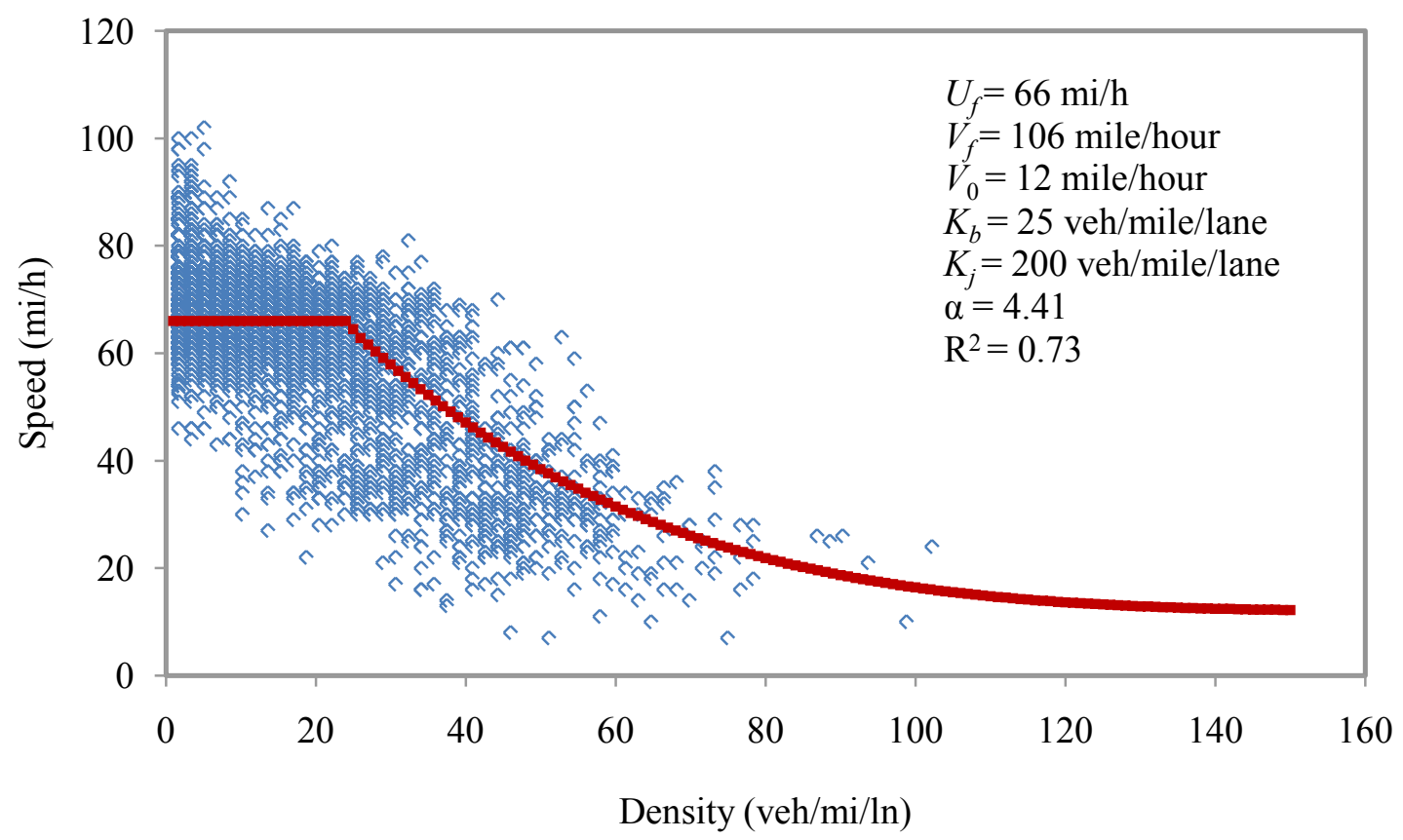

Figure 3.4 Calibration of Type 2 traffic flow model.

Linear regression analysis is a tool to calibrate the above two types of traffic flow models (Mahmassani et al., 2005). This involves transforming the modified Greenshields' model into a linear form and taking the natural logarithm on both sides. For example, the curve part of the Type 1 model can be transformed into Equation 3.4 below:

$$
\operatorname{Ln}\left(V_{i}-V_{0}\right)=\operatorname{Ln}\left(V_{f}-V_{0}\right)+\alpha \operatorname{Ln}\left(1-\frac{K_{i}}{K_{j}}\right)
$$

Based on the fact that the curve passes the point $\left(U_{f}, K_{b}\right)$, the above equation can be further transformed into the following: 


$$
\operatorname{Ln}\left(V_{i}-V_{0}\right)=\operatorname{Ln}\left(U_{f}-V_{0}\right)+\alpha \operatorname{Ln}\left(\frac{K_{j}-K_{i}}{K_{j}-K_{b}}\right)
$$

$U_{f}$ is the free-flow speed of freeway segments, which can be easily deduced from the density-speed scatter plot of a segment. $V_{0}$ and $K_{j}$ can be predefined based on common experience. $K_{b}$ varies for different road segments and can be determined based on the scatter plot of speed and density. Equation 3.5 can then be converted into a simple form of Equation 3.6:

$$
Y=\alpha X+\beta
$$

The parameters in the above equations can be calibrated using the ordinary least square (OLS) method.

\subsection{Initial O-D Matrix Estimation (IODE) Model}

An important part of this study involves estimating an accurate initial O-D matrix to improve the performance of proposed dynamic O-D matrix estimations. The procedure for an initial O-D matrix estimation includes the following steps:

1. Prepare an initial static O-D matrix based on the gravity models,

2. Estimate the static O-D matrix by using a maximum likelihood (ML) estimation model, and

3. Estimate the initial dynamic O-D matrix using a GLS programming method.

The above procedures are described separately in Sections 3.3.1, 3.3.2, and 3.3.3.

\subsubsection{Initial O-D Matrix Preparation}

Gravity models are used to prepare the initial O-D matrix for a study area based on socioeconomic data. Currently, gravity models are the most widely used trip distribution models because they respond better to changes in the network and cost as compared to 
growth factor models, and have less computational burden than intervening opportunity models. For these reasons, gravity models are widely used for trip distribution in transportation planning. An initial O-D matrix can be obtained with the aid of a transportation planning model that implements gravity models during the trip distribution step.

The first rigorous use of a gravity model is by Casey (1955), who suggested using this approach to estimate shopping trips and catchment areas between towns. Since then, the gravity models have been gradually improved. A typical gravity model has the format of Equation 3.7:

$$
T_{i j}=\alpha \mathrm{O}_{i} \mathrm{D}_{j} f\left(c_{i j}\right)
$$

where $T_{i j}$ is the element of trip demand from origin zone $i$ to destination zone $j ; \alpha$ is a proportion factor; $\mathrm{O}_{i}$ is the total production of trips in zone $i$; $\mathrm{D}_{j}$ is the total attraction trips in zone $j$; and $f\left(c_{i j}\right)$ is the deterrence function that represents the disincentive to travel as travel cost $c_{i j}$ increases. Currently, the popular versions for this function are as follows:

$$
\begin{array}{ll}
f\left(c_{i j}\right)=\exp \left(-\alpha c_{i j}\right) & \text { exponential function } \\
f\left(c_{i j}\right)=\left(c_{i j}\right)^{-n} & \text { power function } \\
f\left(c_{i j}\right)=\left(c_{i j}\right)^{-\mathrm{n}} \exp \left(-\alpha c_{i j}\right) & \text { combined function }
\end{array}
$$

In some urban planning models, time-of-day (TOD) factor methods have been developed to convert a daily O-D demand matrix into a peak-period O-D matrix to better replicate the actual traffic conditions in specific periods, such as morning or afternoon peak-hours. For example, the Southeast Regional Planning Model (SERPM) includes TOD modeling to better replicate the variations in travel behavior, traffic congestion, traffic operations, and transit operations throughout the day. With the aid of a gravity 
model in some transportation planning model software packages, a relatively accurate initial O-D matrix, such as a peak-period O-D matrix, can be obtained.

\subsubsection{Static Initial O-D Matrix Estimation (SIODE)}

After a peak-period O-D matrix (about three to four hours) is estimated based on gravity models, the remaining work is to factorize the peak-period matrix into sequential smalltime interval O-D matrices and to estimate each of those small-time interval O-D matrices according to the observed traffic volume data. The factorizing factors can be simply determined based on the distribution pattern of observed traffic volume. The factorized O-D matrix then can be estimated based on some static estimation methods such as maximum likelihood (ML), generalized least square (GLS), and Bayesian inference (BI) models.

In this dissertation, a ML model is used to calibrate the factorized O-D matrices because it is embedded in the CUBE traffic planning software currently in use. The ML O-D estimation model proposed by Spiess (1986) estimates an O-D matrix by using an observed sample O-D matrix, observed link traffic volumes on screenlines and the total productions and attractions of zones. The assumption of the estimation model is an independent Poisson distribution of O-D trips, observed link traffic volumes, and production and attraction trips. The ML O-D matrix estimation model is formulated as Equation 3.8 below:

$$
\begin{aligned}
& M=\min _{T_{i j}}\left\{\sum_{k} \lambda_{Q}\left(V_{k}-Q_{k} \operatorname{Ln}\left(\lambda_{Q} V_{k}\right)\right)+\sum_{i} \lambda_{O}\left(G_{i}-O_{i} \operatorname{Ln}\left(\lambda_{O} G_{i}\right)\right)+\right. \\
& \left.\sum_{j} \lambda_{D}\left(A_{j}-D_{j} \operatorname{Ln}\left(\lambda_{D} A_{j}\right)\right)+\sum_{i j} \lambda_{N}\left(T_{i j}-N_{i j} \operatorname{Ln}\left(\lambda_{N} T_{i j}\right)\right)\right\}
\end{aligned}
$$

subject to: 


$$
\begin{aligned}
G_{i} & =\sum_{j} T_{i j} \\
A_{j} & =\sum_{i} T_{i j} \\
V_{k} & =\sum_{i} \sum_{j} R_{i j k} T_{i j} \\
R_{i j k} & =\text { assignment }\left(T_{i j}\right) \text { based on the static assignment method }
\end{aligned}
$$

where $M$ is the value of an objective function; $k, i$, and $j$ are, respectively, subscripts representing the $k$ th screen line, the $i$ th origin zone, and the $j$ th destination zone; $\lambda_{Q}, \lambda_{O}$, $\lambda_{D}$, and $\lambda_{N}$ are, respectively, confidence factors associated with observed traffic data volume $Q_{k}$, origins trip $O_{i}$, destination trips $D_{j}$, and initial O-D trips $N_{i j} ; V_{k}, G_{i}, A_{j}$, and $T_{i j}$ are, respectively, estimated traffic data volume $V_{k}$, origin trips $G_{i}$, destination trips $A_{j}$, and O-D trips $T_{i j}$; and $R_{i j k}$ is the proportion of trips in O-D matrix cell $(i, j)$ using screenline $k$. $R_{i j k}$ usually needs to be obtained through a static assignment method.

Before performing the ML O-D matrix estimation, two data sets need to be prepared in advance. The first one is observed traffic volumes from roadside detectors. The second one is the sub-period O-D matrix corresponding to each observation time interval. The assumption is that during peak-periods, most travelers have similar traffic behavior (i.e., go to work or go home). Thus, the percentage of traffic volume in a certain time interval over that volume of the peak-period can be used to factorize the peak-period O-D matrix and generate the initial matrices for each time interval. Then, the ML model is used to independently calibrate the initial O-D matrices. In this dissertation, the process of static initial O-D matrix estimation is illustrated in Figure 3.5. 


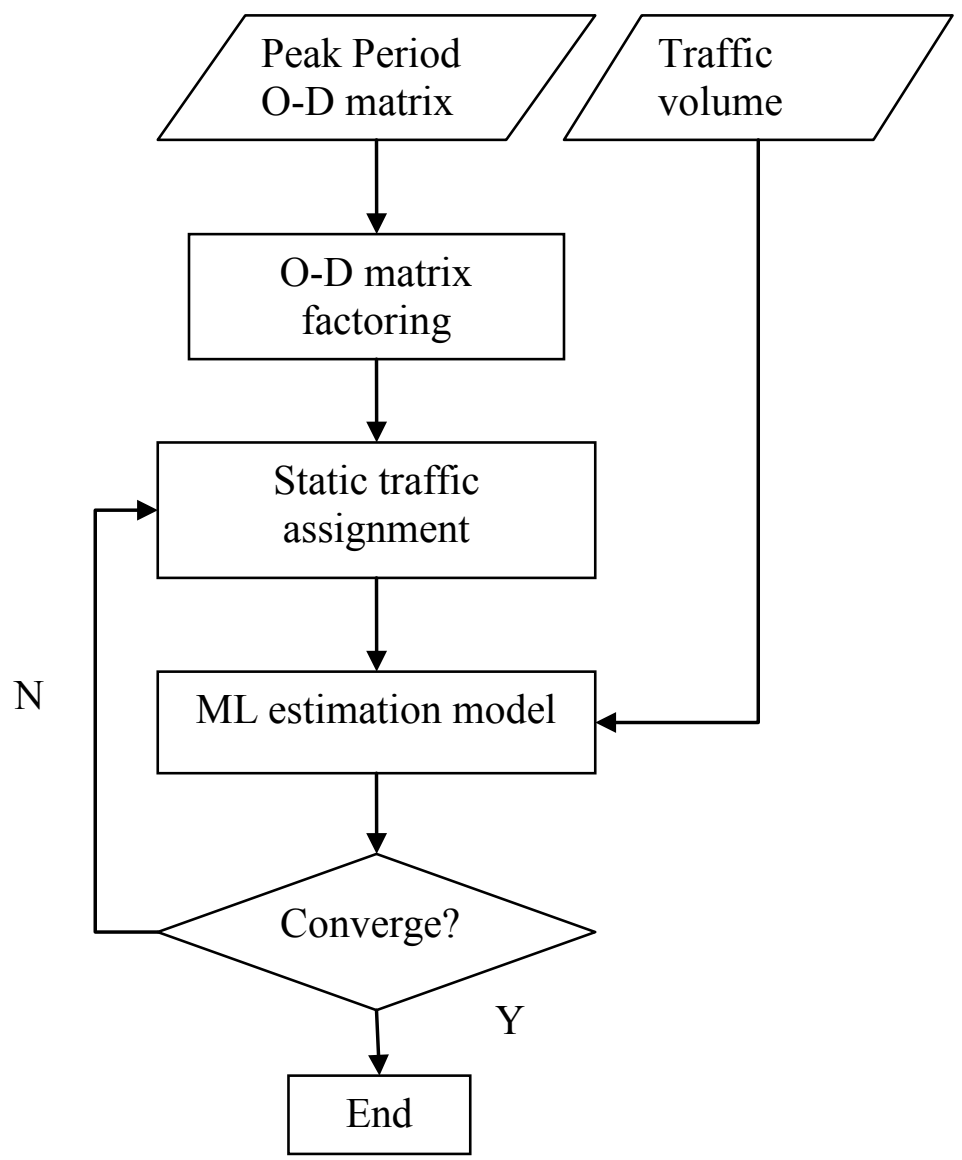

Figure 3.5 Procedure for static initial O-D matrix estimation.

The flowchart in Figure 3.5 can be summarized in the following steps:

1. Factorize the peak hours of the O-D matrix into sub-period O-D matrices;

2. Perform a static user equilibrium traffic assignment to determine the $R_{i j k}$, which is used as the input for the ML model; and

3. Employ the ML model to perform the O-D matrix estimation for each subperiod based on the observed traffic volumes in the corresponding time period. If a convergent result is derived, stop; otherwise, repeat Step 2.

\subsubsection{Dynamic Initial O-D Matrix Estimation (DIODE)}

The SIODE method in the previous section relies on a static assignment, also known as a static route choice. When the estimated O-D matrix is loaded onto the road network by a 
dynamic traffic assignment simulator, there are still large deviations between the assignment and observed traffic measurements (usually link traffic volumes). The deviations include not only random errors but also systematic errors. Typically the deviation distribution can be assumed as Figure 3.6, where the x-axis represents the time interval, and the y-axis stands for the deviation between assignment and observed traffic volumes. Based on the figure, the deviation envelope is far away from the $\mathrm{x}$-axis, so there are systematic deviations in the estimation result. It is necessary to reduce the systematic deviations.

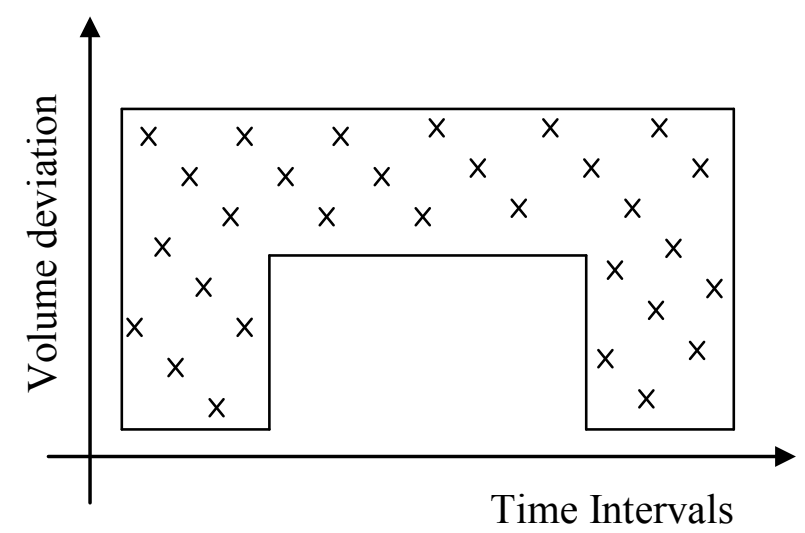

Figure 3.6 The systematic link volume deviation.

The adopted strategy is to reduce the systematic deviations of link volume, while keeping information of the initial input O-D matrix in terms of relative relationship to OD demand values. This can be accomplished by the following two sub-models:

1. Sub-model 1: This model attempts to reduce the systematic deviations of link volume for the entire period by estimating a single scaling factor for the whole time period. The resulting traffic volume deviation distribution is illustrated in Figure 3.7. Since the relative relationship of the O-D demand values remains 
unchanged, there is not much change on the shape of the resulting deviation envelop either.

2. Sub-model 2: This model is designed to obtain an optimal vector of scaling factors to scale the resulting multiple O-D matrices of each time period from Submodel 1. Sub-model 2 tries to reduce the systematic deviation for each time period. The link volume deviation distribution after the estimation is illustrated in Figure 3.8.

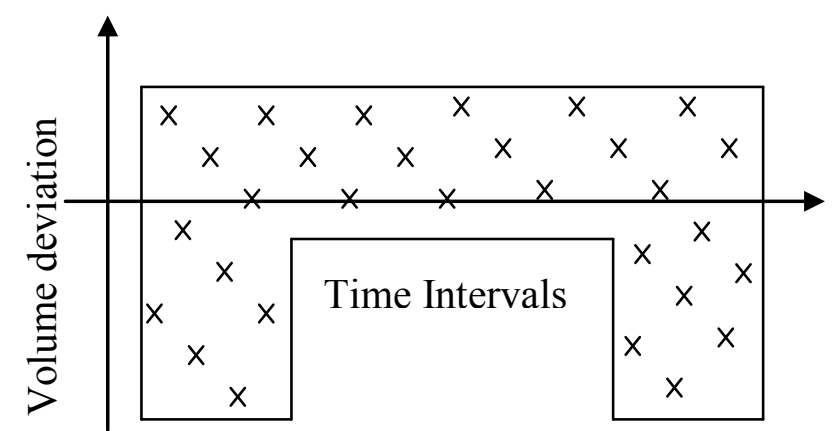

Figure 3.7 The distribution of volume deviation after estimation of Sub-model 1.

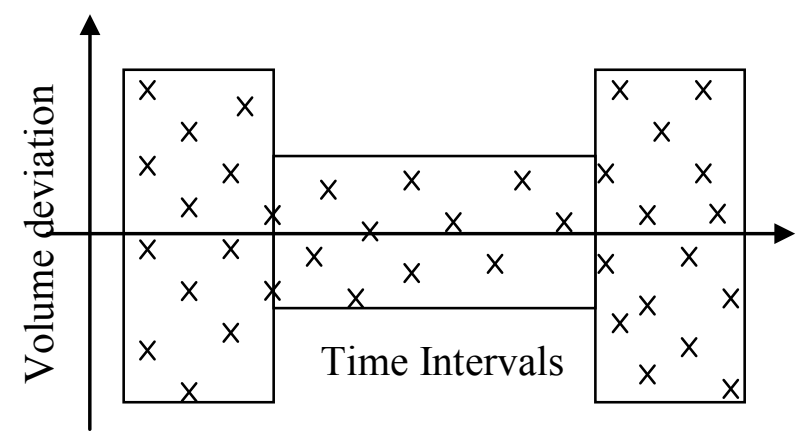

Figure 3.8 The distribution of volume deviation after estimation of Sub-model 2.

Both Sub-models 1 and 2 can be formulated with the mathematical optimization model given as Equation 3.9, below:

Upper-level:

$$
M=\min _{\lambda_{d}}\left\{\sum_{k, t}\left[\left(P_{k, t} \sum_{i, j, d} A_{i, j, d}^{k, t} X_{i, j, d} \lambda_{d}-V_{k, t}^{O}\right) / V_{k, t}^{O}\right]^{2}\right\}
$$

subject to, 


$$
\begin{aligned}
& \lambda_{d} \geq 0 \\
& \lambda_{1}=\lambda_{2}=\cdots=\lambda_{d} \text { (for Sub-model } 1 \text { only) }
\end{aligned}
$$

Low-level:

$$
A_{i, j, d}^{k, t}=\operatorname{assigning} X_{i, j, d} \text { by a DTA simulator }
$$

where $i, j, d, k$, and $t$ are, respectively, subscripts or superscripts for the origin zone, destination zone, departure time interval, link with traffic volume measurement, and observation time interval; $X_{i, j, d}$ is an element of the simulated dynamic O-D matrix; $A_{i, j, d}^{k, t}$ is the link proportion matrix that is proportionate of dynamic O-D matrix $X_{i, j, d}$ passing link $k$ in observation time interval $t ; V_{k, t}^{O}$ represents the observed traffic volume of link $k$ at time interval $t ; \lambda_{d}$ is the factor to be optimized; and $P_{k, t}$ is an adaptive conversion factor for the volume of link $k$ during time interval $t$.

$$
P_{k, t}=1 \text { if } S_{k, t}>S_{k, t}^{C} \text {, and } S_{k, t}^{O}>S_{k, t}^{C} \text { and } P_{k, t}=S_{k, t}^{O} / S_{k, t} \text { if } S_{k, t} \leq S_{k, t}^{C} \text { or } S_{k, t}^{O} \leq S_{k, t}^{C}
$$

where $S_{k, t}, S_{k, t}^{O}$ and $S_{k, t}^{C}$ are the respectively simulated, observed, and critical traffic speed of link $k$ at time interval $t$. The critical traffic speed $S_{k, t}^{C}$ is the link traffic speed that corresponds to the maximum flow rate of link $k$, which is illustrated in Figure 3.9 for the Type 2 traffic flow model. The critical speed $S_{k, t}^{C}$ can be determined for the Type 2 traffic flow model in a similar way. According to the figure, if the traffic speed on link $k$ is greater than the critical speed $S_{k, t}^{C}$, then the traffic condition on link $k$ is uncongested, meaning that an increase of traffic demand in link $k$ will cause the traffic volume to increase as well. If either the observed or simulated traffic speed on link $k$ is less than the 
critical speed, then the traffic condition on link $k$ is congested, and the traffic volume in link $k$ will decrease with the increasing traffic demand.

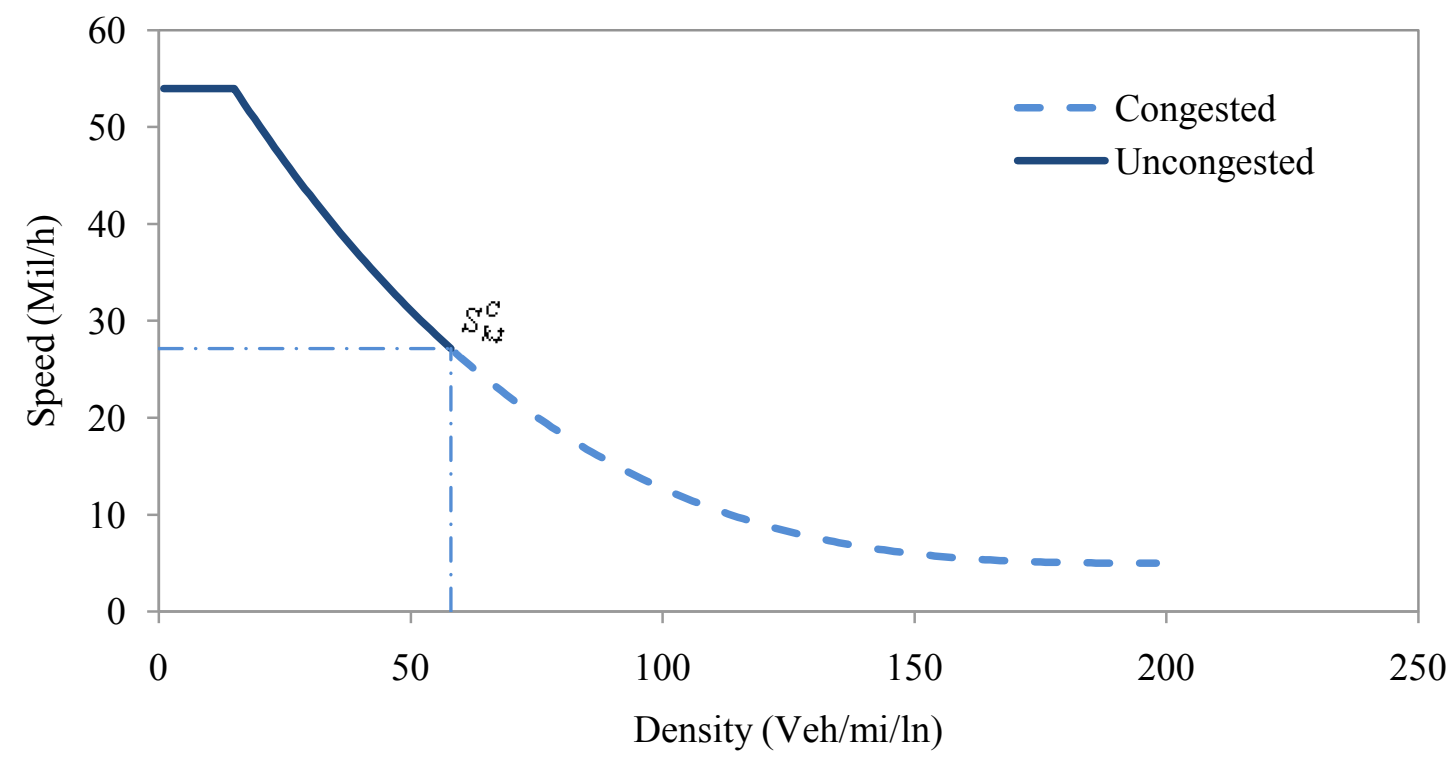

Figure 3.9 Critical speed.

During dynamic O-D estimation, there are two types of traffic congestion: (1) the observed traffic condition is congested, represented by $S_{k, t}^{O}<S_{k, t}^{C}$; and (2) the simulated traffic condition is congested from the inaccurate initial O-D matrix, indicated by $S_{k, t}<$ $S_{k, t}^{C}$. Under these two congested traffic conditions, the adaptive conversion factor $P_{k, t}$ of the proposed models can automatically convert the link traffic volume into the link traffic density, as seen in Equation 3.9, based on the fundamental traffic flow equation. The existing estimation models cannot produce a satisfying result under congested traffic conditions due to the proportional assignment assumption mentioned before. In the proposed model, before estimation, the link traffic condition is evaluated based on $S_{k, t}$, $S_{k, t}^{O}$ and $S_{k, t}^{C}$. If the traffic condition in link $k$ is uncongested both for simulated and observed traffic conditions, then $P_{k, t}=1$, which means that the traffic volume and traffic demand on the link represent a positive relationship, and that the proportional assignment 
assumption works. Otherwise, $P_{k, t}=S_{k, t}^{O} / S_{k, t}$, which means that the proportional relationship is revised by $P_{k, t}$. By substituting $P_{k, t}=S_{k, t}^{O} / S_{k, t}$ into the first term of the objective function and applying the fundamental traffic flow equation $V=S \times D$, Equation 3.10 can be deduced:

$$
\begin{aligned}
M & =\sum_{k, t}\left[\left(\left(S_{k, t}^{O} / S_{k, t}\right) \sum_{i, j, d} A_{i, j, d}^{k, t} X_{i, j, d} \lambda_{d}-V_{k, t}^{O}\right) / V_{k, t}^{O}\right]^{2} \\
& =\sum_{k, t}\left[\left(\sum_{i, j, d}\left(A_{i, j, d}^{k, t} X_{i, j, d} \lambda_{d}\right) / S_{k, t}-V_{k, t}^{O} / S_{k, t}^{O}\right) /\left(V_{k, t}^{O} / S_{k, t}^{O}\right)\right]^{2} \\
& =\sum_{k, t}\left[\left(\sum_{i, j, d} D_{k, t}-D_{k, t}^{O}\right) / D_{k, t}^{O}\right]^{2}
\end{aligned}
$$

where $D_{k, t}^{o}$ and $D_{k, t}$ are observed and estimated traffic densities for link $k$ during time interval $t$.

The link volume is transformed into a density measurement in the proposed model with the aid of $P_{k, t}$. The relationship between density and traffic demand is positive and monotonic, thus the converted objective function in Equation 3.10 works for congested traffic conditions. By optimizing the $\lambda_{i}$ factor through Equation 3.9, a better initial dynamic O-D matrix can be obtained.

The solution algorithm for the DIODE model can be illustrated as the following flowchart Figure 3.10. The detailed procedure to perform DIODE is presented as follows:

1. Based on the initial O-D matrix and road network, the DTA simulator is used to perform a DTA simulation.

2. From the simulation result, the link proportional matrix $\mathbf{A}$ is extracted. 
3. The adaptive conversion vector $\mathbf{P}$ is updated based on the comparison between the simulated speed and the critical speed, and between the observed speed and the critical speed.

4. The objective function is constructed based on the available data set, and can be solved by a nonlinear mathematical programming method.

5. If the estimated O-D matrix can decrease the objective function value, then go to Step 1 to perform another round of estimation; otherwise, the estimation is terminated.

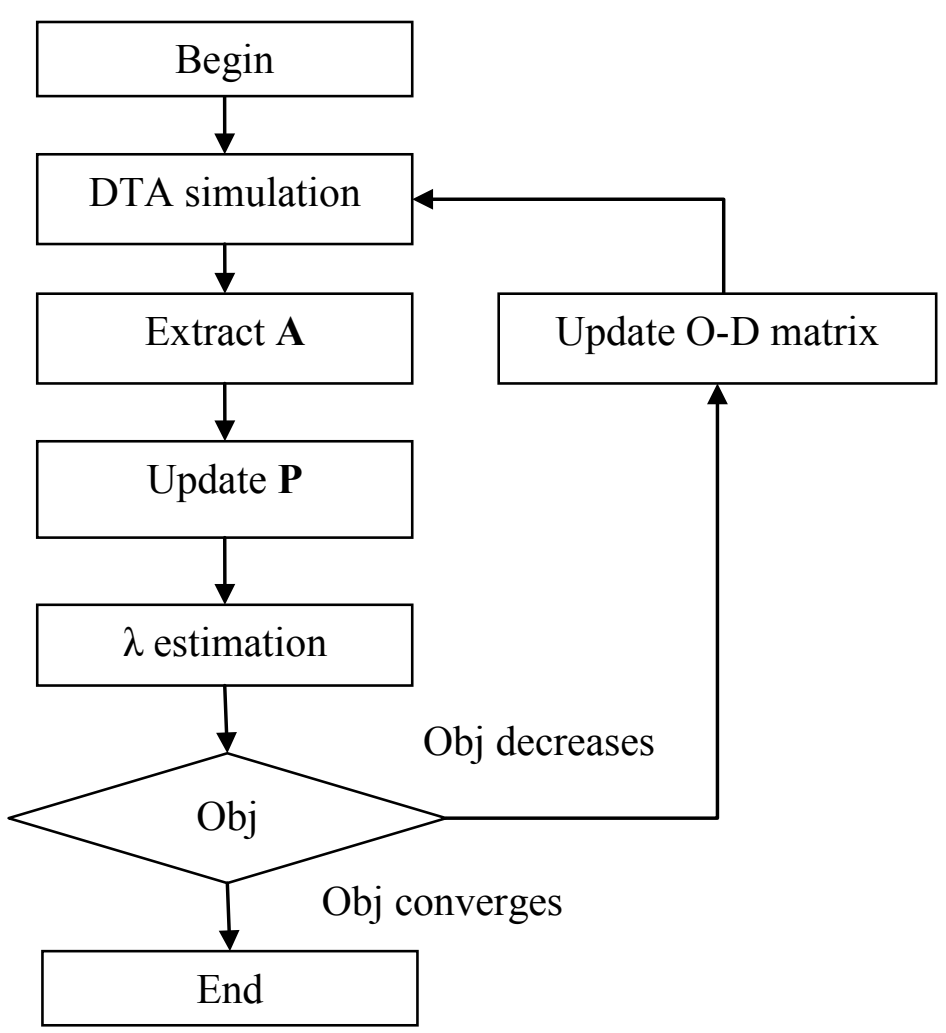

Figure 3.10 The procedure to perform DIODE.

\subsection{Dynamic O-D Matrix Estimation (DODE)}

The proposed dynamic O-D matrix estimation (DODE) model is an extension of the bilevel dynamic O-D demand estimation model proposed by Zhou et al. (2004). 
Specifically, the upper-level is a constrained ordinary least squares (OLS) model that aims to estimate dynamic O-D demands based on observed traffic volumes and link choice proportion matrices. A link choice proportion matrix is generated from the dynamic traffic network loading model in the lower-level. The DODE model is shown as Equation 3.11 below:

Upper-level:

$$
\begin{aligned}
& M=\min _{X_{i, j, d}}\left\{(1-w) \sum_{k, t}\left[\left(P_{k, t} \sum_{i, j, d} A_{i, j, d}^{k, t} X_{i, j, d}-V_{k, t}^{O}\right) / V_{k, t}^{O}\right]^{2}\right. \\
& +w \sum_{i, j, d}\left[\left(X_{i, j, d}-g\left(X_{i, j, d-1}, X_{i, j, d-2}, \cdots, X_{i, j, d-q}\right)\right) / X_{i, j, d}\right]^{2} \\
& \left.+w \sum_{i, j, d}\left[\left(X_{i, j, d}-X_{i, j, d}^{O}\right) / X_{i, j, d}^{O}\right]^{2}\right\}
\end{aligned}
$$

subject to,

$$
X_{i, j, d} \geq 0
$$

$$
\begin{aligned}
& P_{k, t}=1 \text { if } S_{k, t}>S_{k, t}^{C} \text { and } S_{k, t}^{O}>S_{k, t}^{C} \text { and } P_{k, t}=S_{k, t}^{O} / S_{k, t} \text { if } S_{k, t} \leq S_{k, t}^{C} \text { or } S_{k, t}^{O} \leq S_{k, t}^{C} \\
& \mathrm{~g}\left(X_{i, j, d-1}, X_{i, j, d-2}, \ldots, X_{i, j, d-q}\right)=\beta_{0}+\beta_{1} X_{i, j, d-1}+\beta_{2} X_{i, j, d-2}, \ldots+\beta_{q} X_{i, j, d-q}
\end{aligned}
$$

Low-level:

$$
A_{i, j, d}^{k, t}=\operatorname{assigning} X_{i, j, d} \text { based on a DTA simulator }
$$

where $i, j, d, k$, and $t$ are, respectively, subscripts or superscripts for the origin zone, destination zone, departure time interval, link with traffic volume measurement, and observation time interval; $X_{i, j, d}$ and $X_{i, j, d}^{O}$ are the elements of the estimated dynamic O-D matrix and that of the initial O-D matrix, respectively; $A_{i, j, d}^{k, t}$ is the link proportion matrix, which is the proportion of dynamic O-D matrix $X_{i, j, d}$ that passes link $k$ during observation time interval $t ; V_{k, t}^{O}$ is the observed traffic volume on link $k$ during time interval $t ; P_{k, t}$ is 
an adaptive conversion factor for the volume on link $k$ during time interval, and $P_{k, t}=1$ if $S_{k, t}>S_{k, t}^{C}$ and $S_{k, t}^{O}>S_{k, t}^{C}$ and $P_{k, t}=S_{k, t}^{O} / S_{k, t}$ if $S_{k, t} \leq S_{k, t}^{C}$ or $S_{k, t}^{O} \leq S_{k, t}^{C} ; S_{k, t}, S_{k, t}^{O}$, and $S_{k, t}^{C}$ are, respectively, the simulated, observed, and critical traffic speeds of link $k$ during time interval $t$; the constant $w$ is an adaptive weighting factor that automatically changes the weight on the demand adjustment term; and $\mathrm{g}()$ stands for the auto regression relationship between $X_{i, j, d-1}, X_{i, j, d-2}, \ldots, X_{i, j, d-q}$; and coefficient $\beta_{i}$ needs to be calibrated based on historical demand data. The critical traffic speed $S_{k, t}^{C}$ is the link traffic speed corresponding to the maximum flow rate for link $k$, which is illustrated in Figure 3.9.

The objective function in Equation 3.11 contains three terms: the deviation between the observed and simulated link volumes, the deviation between target and estimated traffic demand transition patterns, and the deviation between target and estimated traffic demands. The functions of the three terms are listed as follows:

1. The link traffic volume deviation term is used to match the estimated link volume to an observed one by adjusting the entries of a dynamic O-D matrix based on the link choice proportion matrix $A_{i, j, d}^{k, t}$. In order to improve the estimation performance under congested traffic conditions, a conversion factor $P_{k, t}$ is integrated into this term, which has been explained in Equation 3.10.

2. The traffic demand transition term is used to preserve the known temporal distribution pattern of the initial input O-D matrix. The traffic demand transition deviation term has not been used widely in previous GLS-based dynamic O-D estimation models. Balakrishna et al., (2007) show that the traffic demand 
transition deviation term can improve the performance of dynamic O-D matrix estimation models.

3. The traffic demand deviation term is the third term employed to restrict the magnitude of the adjustment on traffic demand in an attempt to avoid overadjustment of the O-D demand.

There are three weighting factors assigned to these three deviation terms in the objective function. Those factors are (1-w), w, and $w$ which represent the relative importance of the three deviation terms; the more important the term, the larger the weighting factor. For the purposes of simplification, the model for Equation 3.11 uses an equal weight factor $w$ on the transition deviation term and demand deviation terms. In reality, the weight of the two terms can be determined based on practical experience. In the proposed model, an adaptive $w$ is used to restrict the adjustment on O-D demand in the case of a poor estimation result. The reason for using an adaptive $w$ will be presented later. Compared with existing dynamic O-D matrix estimation models, the proposed model has three advantages in combining adaptive conversion factor $P_{k, t}$, adaptive weighting factor $w$, and the transition term.

The first advantage is when traffic congestion occurs on link $k, P_{k, t}$ can automatically convert the link traffic volume deviation into the link traffic density deviation in the objective function of Equation 3.11. Thus, it can produce better results than existing models with the proportional assignment assumption.

The second advantage is that adaptive weighting factor $w$ is employed in this study to better restrict the magnitude of O-D adjustments in each round of iteration. In the existing GLS-based dynamic O-D matrix estimation models, the weighting factor $w$ is 
fixed. It reflects the relative confidence level of the deviation terms of the objective function and can be estimated through the GLS method (Cascetta, 1984). Zhou et al. (2004) suggested incorporating the planner's knowledge and experience in the weighting factor during estimation and proposed an interactive method to determine the best compromise between weight factors for the dynamic O-D matrix estimation problem. There are two reasons for adopting the adaptive weighting factor $w$ in this study:

1. At the beginning of estimation, a large weight should be assigned to the traffic measurement deviation term in the objective function because a high confidence should be imputed to the traffic data collected from the real world. With the increase of the estimation iteration, when better estimation results are derived, more weight should be assigned to the demand deviation term and the transition deviation term of the objective function because there is higher confidence in the estimated O-D demand.

2. The upper-level estimation model adjusts the O-D demand based on the constant link proportion $A_{i, j, d}^{k, t}$. Since link proportion $A_{i, j, d}^{k, t}$ is naturally related to traveler's route choice behaviors, an O-D demand adjustment can affect the traveler's route choice and thus change link proportions $A_{i, j, d}^{k, t}$. There is circular dependency between O-D demand and link proportion $A_{i, j, d}^{k, t}$; therefore, by adjusting the O-D demand with a fixed link proportion $A_{i, j, d}^{k, t}$, the upper-level model may run the risk of divergence, meaning that too much O-D matrix adjustment can cause a drastic change in the link proportion and result in an increased objective value for the next round of iteration. The adaptive weighting factor $w$, however, can restrict the 
magnitude of an adjustment to the O-D demand. If a divergence problem arises in an iteration, $w$ is automatically increased to reduce the magnitude of O-D demand adjustment for the next iteration. The adaptive weighting factor $w$ can enhance the efficiency of the dynamic O-D matrix estimation and reduce unnecessary iterations. It is worthwhile to mention that $X_{i, j, d}^{O}$ in Equation 3.11, as the element of the target O-D matrix, should be updated for every iteration with a better estimated $X_{i, j, d}$.

The third advantage is that the proposed estimation model can combine the historical or known temporal distribution information of O-D demand into the estimation by using the transition term. Balakrishna et al. (2007) show that the traffic demands transition deviation term can improve the performance of dynamic O-D matrix estimation models. The solution algorithm for the DODE model is illustrated using the flowchart in Figure 3.11. 


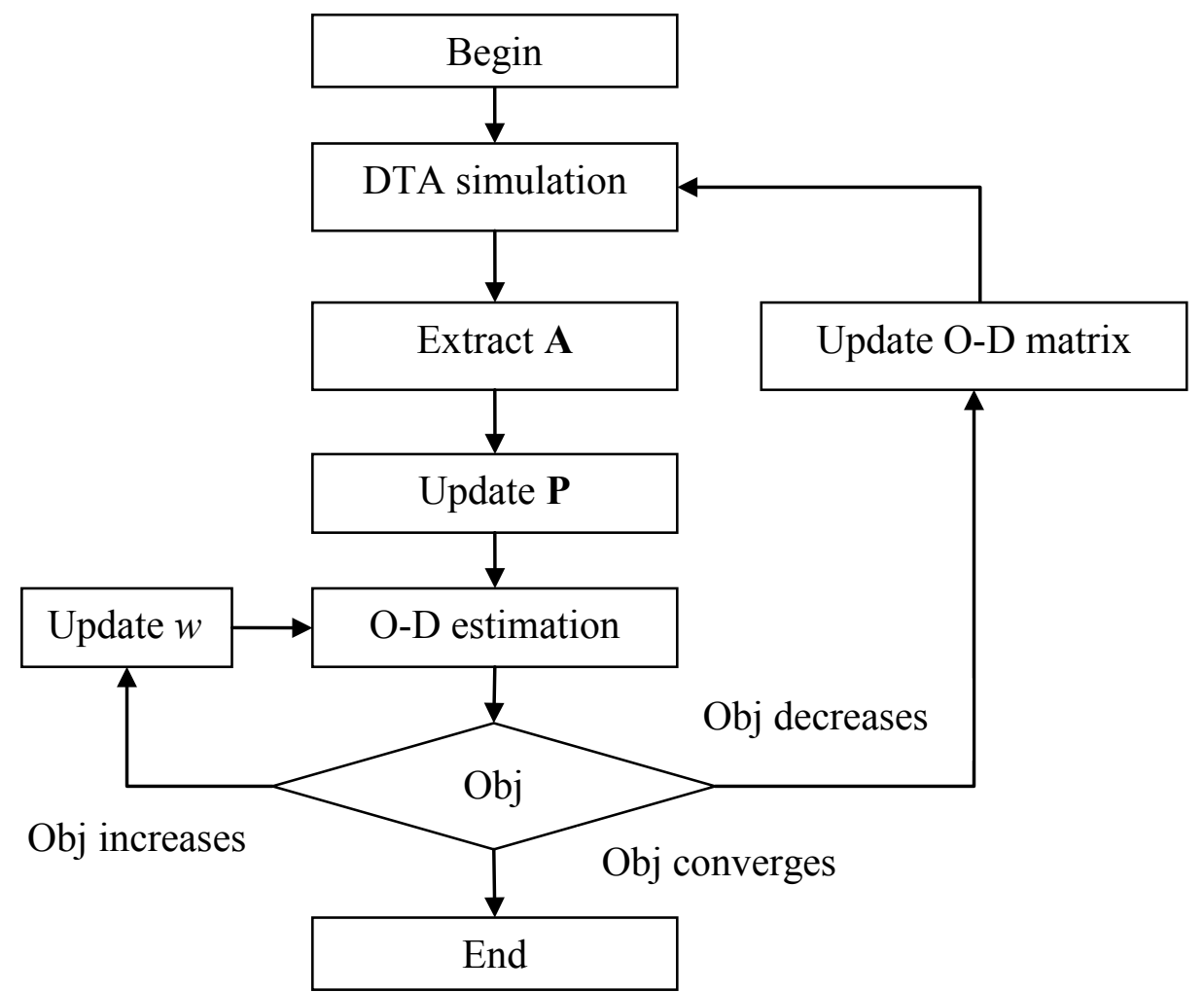

Figure 3.11 The procedure to perform DODE.

The detailed procedure to perform DODE is presented as follows:

1. $w$ is initialized as a certain value and its step is predefined as $\alpha$, for example. Based on the initial O-D matrix and road network, the DTA simulator is used to perform a DTA simulation.

2. From the simulation result, the link proportional matrix $\mathbf{A}$ is extracted.

3. The adaptive conversion vector $\mathbf{P}$ is updated based on the comparison between the simulated speed and the critical speed, and between the observed speed and the critical speed.

4. The objective function is constructed based on the available data set, and can be solved by a nonlinear mathematical programming method. 
5. If the estimated O-D matrix decreases the objective function value then go to Step 1 to perform another round of estimation; if the estimated O-D matrix increases the objective function value, $w=w+\alpha$, then go to Step 4 to solve the objective function again with the updated $w$; and if the $w$ reaches the predefined threshold or the predefined maximum iteration number has been reached, then the estimation is terminated.

In the above mentioned algorithms, for the DIODE and DODE model, the General Algebraic Modeling System (GAMS) software package (Murtagh and Saunders, 1987) is used to solve the nonlinear programming problems of Equations 3.10 and 3.11. GAMS is easy to use and powerful in solving large scale linear/nonlinear programming problems.

The originality of the DIODE and DODE model is in that these two models can automatically convert the deviation of traffic volume into the deviation of converted traffic density in the objective function, in congested link traffic conditions. Compared with the model of Zhou et al. (2005), the models do not completely replace the traffic volume deviation with traffic density deviation. This is because, in real word, traffic density data are not directly available and need to be converted from traffic occupancy data. The conversion may introduce some uncertain errors into the final converted traffic density because the conversion function assumes that the vehicles are evenly distributed on a road link, and the conversion function also needs some unavailable data such as average vehicle length. In order to reduce the errors in traffic measurement data, the proposed DIODE and DODE models do not use converted traffic density deviation unless necessary (under congested traffic conditions). In addition, by converting the traffic 
volume deviation into traffic density deviation, the traffic speed data are implicitly incorporated into the estimation model, which means more traffic information can be combined into the estimation models, thus contributing to the improvement of estimation performance.

\subsection{Traffic Flow Model Fine-Tuning (TFMFT) Model}

Because there may be some errors in the collected traffic data, the traffic flow model calibration (TFMC) cannot guarantee good traffic flow models. In order to reduce the deviation between the assigned traffic measurement and observed traffic measurement, traffic flow models need to be fine-tuned.

The developed traffic flow model fine-tuning (TFMFT) model has a bi-level structure. Specifically, the upper-level is a constrained, OLS model that can minimize the combined deviation between observed and simulated traffic measurements. A DTA simulator is used to produce simulated traffic measurements at the lower-level. The upper-level model can be presented as Equation 3.13 below:

$$
M_{2}=\min _{\theta}\left\{w_{1} \sum_{k, t}\left[\left(V_{k, t}-V_{k, t}^{O}\right) / V_{k, t}^{O}\right]^{2}+\sum_{k, t}\left[\left(S_{k, t}-S_{k, t}^{O}\right) / S_{k, t}^{O}\right]^{2}\right\}
$$

subject to,

$$
\theta \in \Theta
$$

where $M_{2}$ is the objective function value; $\theta$ is the parameter set of the traffic flow models; $\Theta$ is the domain of parameter values; $V_{k, t}$ and $S_{k, t}$ are outputs of the DTA simulator; $w_{1}$ is a weighting factor which represents the relative importance of the two deviation terms in the objective function; and $w 1$ can be determined with the practical experience. 
In the model shown in Equation 3.13, the objective function has two terms. One represents traffic volume deviation and the other signifies traffic speed deviation. An adaptive weighting factor $w_{1}$ is introduced to balance the weighting of the two deviation function terms and to increase the chance that these two terms will decrease simultaneously.

This study uses a stochastic algorithm to calibrate the parameters of traffic flow models because there is no explicit relationship between traffic measurements and the parameters of traffic flow models.

Simultaneous perturbation stochastic approximation (SPSA) (Spall, 1998) can solve the above model by estimating the gradient vector using only two objective function evaluations. The objective function evaluation in this study attempts to perform a DTA simulation with the updated traffic flow models. The minimization process of SPSA can be described in Equations 3.14 and 3.15:

$$
\theta_{i+1}=\theta_{i}-\mathrm{a} i \hat{\mathrm{g}}\left(\theta_{i}\right)
$$

where $\theta_{i}$ is the parameter vector in iteration $i$; $\hat{\mathrm{g}}\left(\theta_{i}\right)$ is an estimation of the gradient vector; and a $i$ is a gain factor. The gradient vector can be calculated using the following equation:

$$
\hat{g}\left(\boldsymbol{\theta}^{i}\right)=\operatorname{Diag}\left[\Delta_{i 1}^{-1}, \Delta_{i 2}^{-1}, \cdots, \Delta_{i k}^{-1}\right] \frac{z\left(\boldsymbol{\theta}_{i}+c_{i} \boldsymbol{\Delta}_{i}\right)-z\left(\boldsymbol{\theta}_{i}-c_{i} \boldsymbol{\Delta}_{i}\right)}{2 c_{i}}
$$

where $\mathrm{z}()$ is the objective function; $c_{i}$ is a decreasing sequence of small, positive numbers; $\Delta_{i}$ is a $k$-dimensional perturbation vector consisting of component-wise 
perturbations represented by $\Delta_{i k}$; and $\operatorname{Diag}[\cdot]$ transforms the input elements into a diagonal matrix.

Since the simultaneous calibration of traffic models is time-consuming for vectors of a large dimension, this study adopts a successive strategy to calibrate the traffic flow models one-by-one, instead of calibrating them all together as in the study by Balakrishna et al. (2007). The successive calibration steps are as follows:

1. The link with maximum speed deviation is selected, and the parameters of the corresponding traffic flow model are calibrated using the proposed method.

2. When the objective value does not decrease within a certain consecutive number of iterations, go to Step 1 to find the next traffic flow model to be calibrated.

3. If the maximum iteration number is reached, terminate the procedure.

The successive strategy imitates the intuitive manner in which this calibration problem would be handled manually. The successive strategy can effectively cut down the dimension of the vector of unknown variables for the stochastic algorithm, thus reducing the number of iterations significantly.

\subsection{Summary}

In this chapter, a complete methodology framework is proposed to solve the stated problems. Compared with existing dynamic O-D estimation models, the proposed methodology framework has two advantages over the existing dynamic O-D estimation models: (1) it can produce a high confidence level for the initial O-D matrix, which has the potential to significantly improve the accuracy of dynamic O-D estimation and reduce the associated computation time; (2) the framework can automatically convert traffic volume deviation to traffic density deviation in the objective function, under the 
congested traffic conditions, to avoid the side impact from the proportional assignment assumption of the existing dynamic O-D estimation models. In addition, by converting the traffic volume data into traffic density data, the traffic speed data can be implicitly incorporated into the estimation model, which means more traffic information can be incorporated into the estimation model overall, thus contributing to the improvement of estimation performance.

The methodology framework is composed of four parts, namely the traffic flow model calibration (TFMC) model, the initial O-D estimation (IODE) model, the dynamic O-D estimation (DODE) model, and the traffic flow model fine-tuning (TFMFT) model. The TFMC model aims to calibrate the supply-side parameters of the DTA model in order to ensure that the speed-density relationship in the DTA simulator mirrors real situations. TFMC uses a linear regression to calibrate the supply-side parameters.

The IODE model aims to provide an accurate input O-D matrix for the DODE model to estimate results. The IODE is comprised of two parts: the static initial O-D matrix estimation (SIODE) model and the dynamic initial O-D matrix estimation (DIODE) model. The first part attempts to perform a static O-D matrix estimation based on a maximum likelihood (ML) estimation method. The second part further calibrates the resulting O-D matrix from the first part based on a GLS estimation model.

The DODE model performs an enhanced dynamic O-D matrix estimation, especially in congested traffic conditions. Unlike the poor performance of existing estimation models using the problematic proportional assignment under congested traffic conditions, the DODE model can automatically detect the link with congested traffic conditions. Furthermore, the DODE method can go on to incorporate the speed data into 
the model to revise the link proportion matrix in the estimation. Based on the theoretic analysis, this model can improve dynamic O-D matrix estimations.

The TFMFT model further adjusts the parameters in the traffic flow models of the DTA simulator in order to match the simulated speed to the observed speed. However, there may be outlier data from the detectors; as such, the TFMC model cannot produce the best-fitted traffic flow models, and thus the TFMFT model is used. The successive SPSA algorithm is used to perform the TFMFT. When the size of the road network becomes large, it will take too long a time for the successive SPSA algorithm to perform a TFMFT. Thus, TFMFT is not a recommended method for this situation. 


\section{CHAPTER 4}

\section{A SYNTHETIC CASE STUDY}

In this chapter, only the proposed dynamic O-D matrix estimation (DODE) model is applied to a hypothetical road network. The performance of the proposed dynamic O-D matrix estimation model is compared with that of a typical estimation model (Zhou et al., 2003), shown in Equation 2.24. Zhou's model is a classic bi-level dynamic O-D estimation model with a GLS optimization in the upper-level and with dynamic traffic assignment in the lower-level. Because Zhou's model has a structure and input similar to the proposed dynamic O-D estimation model, Zhou's model is chosen as the benchmark for comparison with the proposed model in order to clearly analyze the effect of each term in the proposed dynamic O-D estimation model. Section 4.1 introduces the configuration of the road network and traffic data; Section 4.2 implements the proposed DODE model; and Section 4.3 compares the performance of the proposed model with that of Zhou's model.

\subsection{Road Network Configuration}

The case study is performed on a hypothetical freeway network and shown in Figure 4.1. The total number of O-D pairs is $1 \times 1$ (only $1 \rightarrow 2$ ). A detector is coded in the lower branch of the road network, and it collects traffic volume and speed data for each simulation time interval of 15 minutes. There are 8 simulation time intervals in total. 


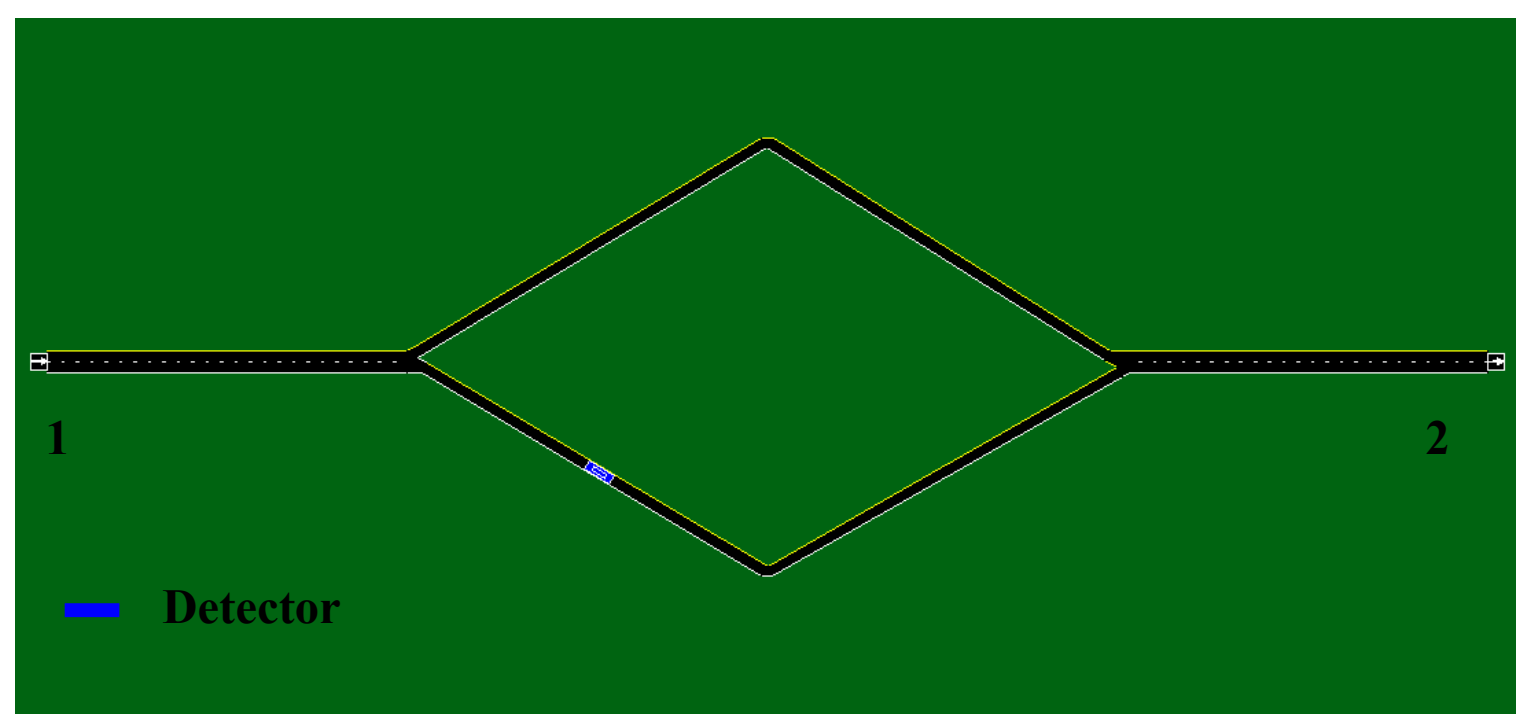

Figure 4.1 A hypothetical freeway road network.

Two assumptions are made in this case study:

1. An assumed traffic flow model is used for the hypothetical freeway network's links. The employed traffic flow model is illustrated in Figure 4.2.

2. The real and initial O-D demands from Zone 1 to Zone 2, for each time interval, are shown in Table 4.1. The initial O-D matrix estimation model proposed in Chapter 3 on methodology is not used. In order to show the capability of a DODE model under initial congested conditions, the initial O-D matrix is set much higher than what the road network can support. For the purpose of simplicity, the elements of the O-D matrix, for all time intervals, are assumed to be equal. 


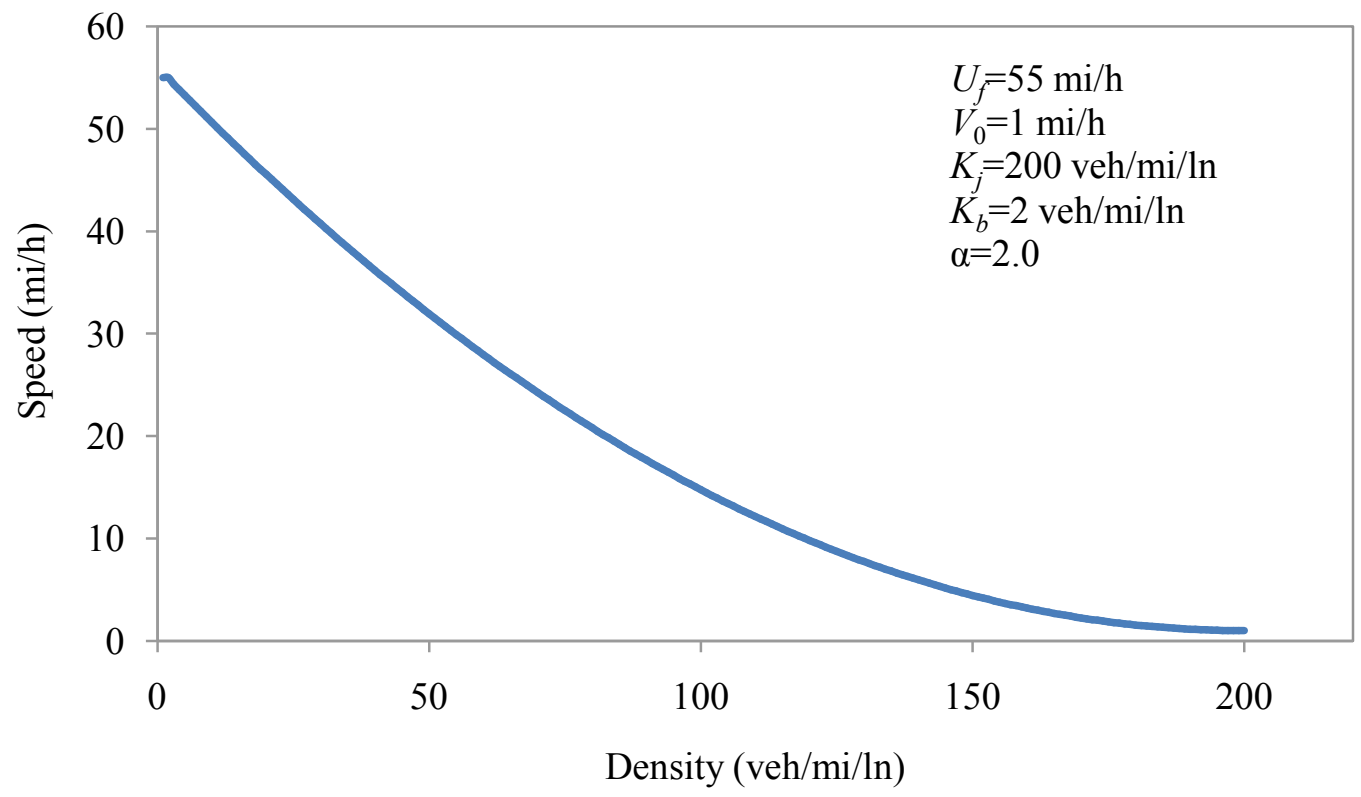

Figure 4.2 Assumed traffic flow model.

Table 4.1 Real and initial O-D demand.

\begin{tabular}{|l|l|l|l|l|l|l|l|l|}
\hline Time Intl. & 1 & 2 & 3 & 4 & 5 & 6 & 7 & 8 \\
\hline Real & 600 & 600 & 600 & 600 & 600 & 600 & 600 & 600 \\
\hline Initial & 850 & 850 & 850 & 850 & 850 & 850 & 850 & 850 \\
\hline Difference & $41.7 \%$ & $41.7 \%$ & $41.7 \%$ & $41.7 \%$ & $41.7 \%$ & $41.7 \%$ & $41.7 \%$ & $41.7 \%$ \\
\hline
\end{tabular}

With the hypothetical road network and the assumed O-D matrix and traffic flow model, the DTA simulator is used to dynamically load the available O-D matrix onto the road network. After simulation, the traffic measurement data from the detector was extracted, as demonstrated by Figures 4.3 and 4.4.

Figure 4.3 shows the simulated traffic speed when the real demand and initial demand are loaded onto the road network. Based on Figure 4.3, the initial O-D matrix causes heavy traffic congestion on the link with a detector, and the speed on the link decreases rapidly after the first time interval. Figure 4.4 shows the simulated traffic volume, which reflects the same congested traffic situation indicated in Figure 4.3. 


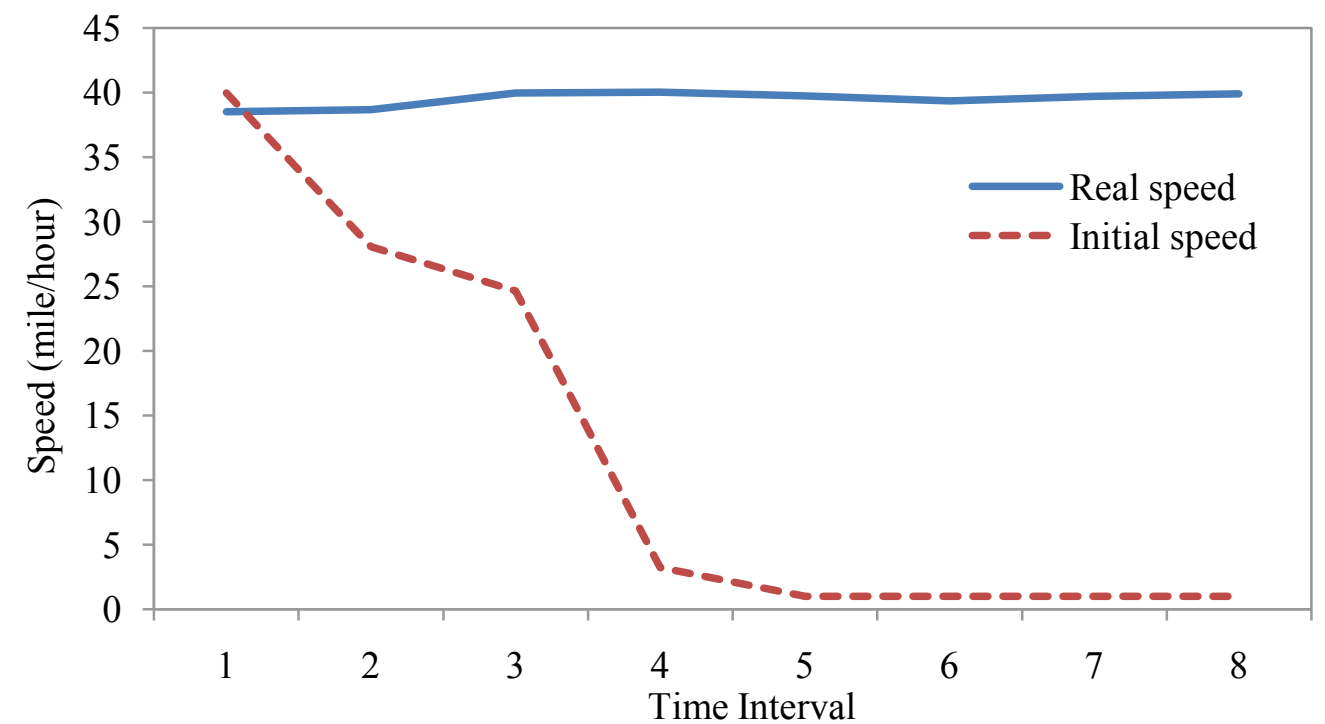

Figure 4.3 Real and initial simulated traffic speed from the detector.

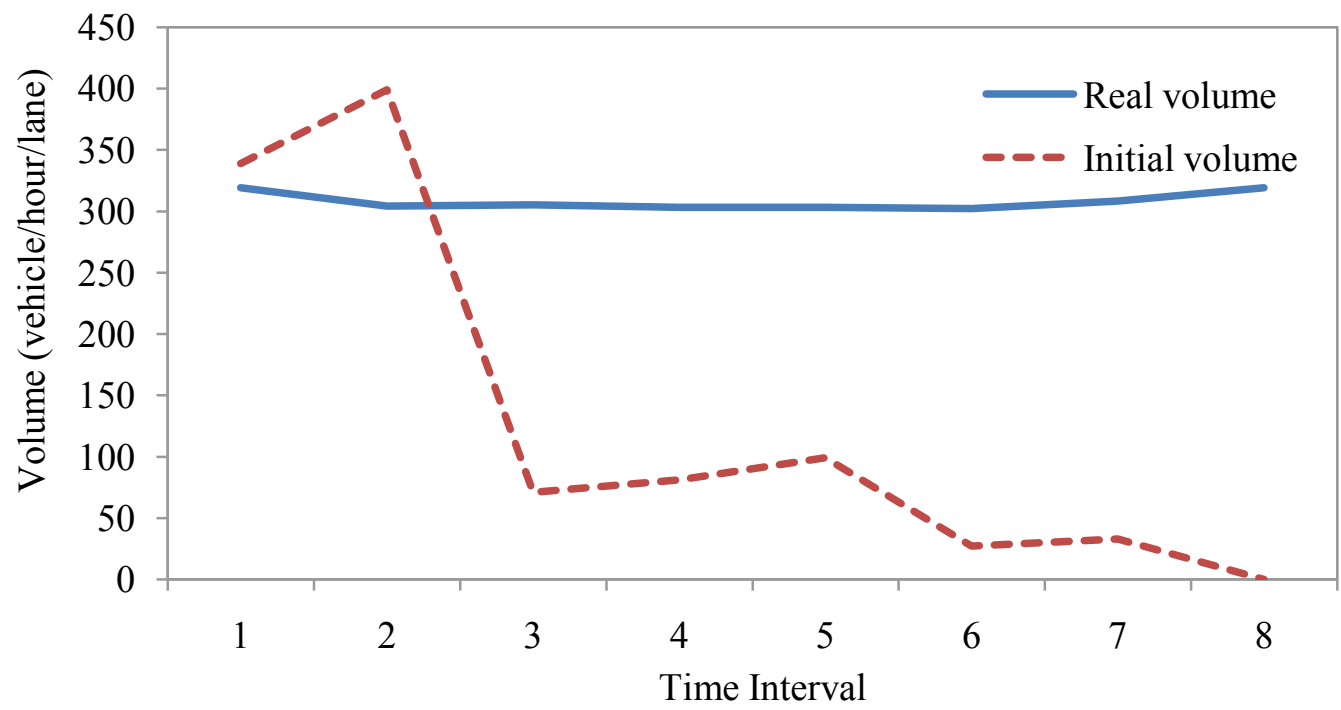

Figure 4.4 Real and initial simulated traffic volume from the detector.

Detailed information on simulation speed and volume is given in Table 4.2 and

Table 4.3 below.

Table 4.2 Real and initial traffic speed

\begin{tabular}{|l|l|l|l|l|l|l|l|l|}
\hline Time Intl. & 1 & 2 & 3 & 4 & 5 & 6 & 7 & 8 \\
\hline Real & 38.5 & 38.65 & 39.96 & 40.03 & 39.73 & 39.36 & 39.72 & 39.91 \\
\hline Initial & 39.95 & 28.05 & 24.62 & 3.23 & 1 & 1 & 1 & 1 \\
\hline Difference & $4 \%$ & $-27 \%$ & $-38 \%$ & $-92 \%$ & $-97 \%$ & $-97 \%$ & $-97 \%$ & $-97 \%$ \\
\hline
\end{tabular}


Table 4.3 Real and initial traffic volume

\begin{tabular}{|l|l|l|l|l|l|l|l|l|}
\hline Time Intl. & 1 & 2 & 3 & 4 & 5 & 6 & 7 & 8 \\
\hline Real & 319 & 304 & 305 & 303 & 303 & 302 & 308 & 319 \\
\hline Initial & 339 & 399 & 71 & 81 & 99 & 27 & 33 & 0 \\
\hline Difference & $6 \%$ & $31 \%$ & $-77 \%$ & $-73 \%$ & $-67 \%$ & $-91 \%$ & $-89 \%$ & $-100 \%$ \\
\hline
\end{tabular}

\subsection{Dynamic O-D Matrix Estimation (DODE)}

In this case study, only the dynamic O-D matrix estimation (DODE) model is used to perform the estimation. This investigation will test the three terms integral to estimation model; namely the adaptive weighting factor, the adaptive conversion factor, and the transition term, respectively.

Firstly, the proposed DODE model is employed to perform the estimation. The performance of the model is illustrated in Figure 4.5.

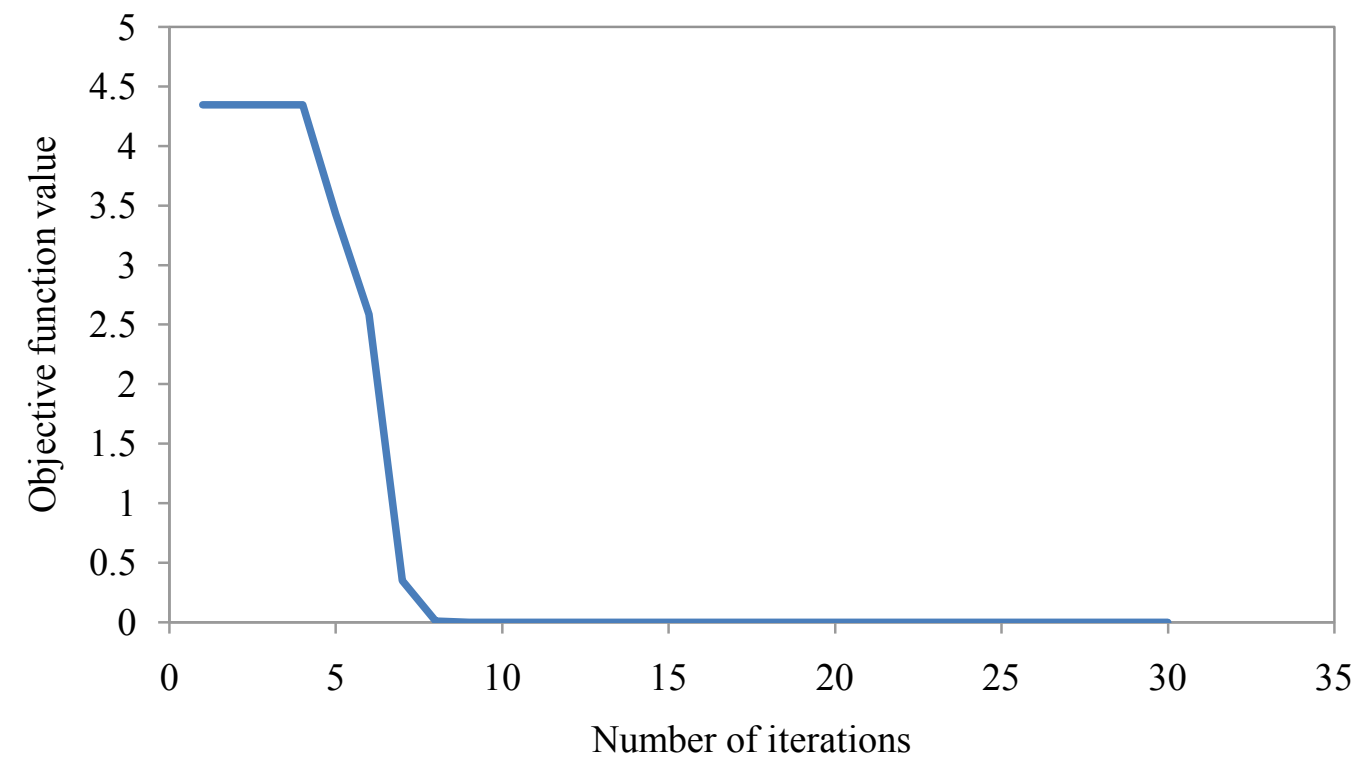

Figure 4.5 Performance of the proposed dynamic O-D matrix estimation model.

According to Figure 4.5, the model demonstrates a strong capability in dynamic O-D matrix estimation. After 8 iterations, the objective function value drops from 4.345 to 0.008 and reaches the minimum solution point. 
It is worth mentioning that there is a plateau in the first 5 iterations of Figure 4.5, which means that the objective function value does not decrease in these iterations. Figure 4.6 shows a comparison among real demand, initial demand, and the estimated demand after the first iteration. According to the figure, at the beginning, the model tends to overact to the congested traffic condition, and the estimated O-D demand is much less than the initial and real O-D demand. Under this circumstance, the weighting factor $w$ in Equation 3.11 is increased automatically, which means more weight is needed to be assigned to the demand term of the objective function in order to reduce the adjustment magnitude of the dynamic O-D demand in the next iteration. The adjustment of the weighting factor will not terminate until the objective function value decreases after the fifth iteration, as illustrated in Figure 4.5.

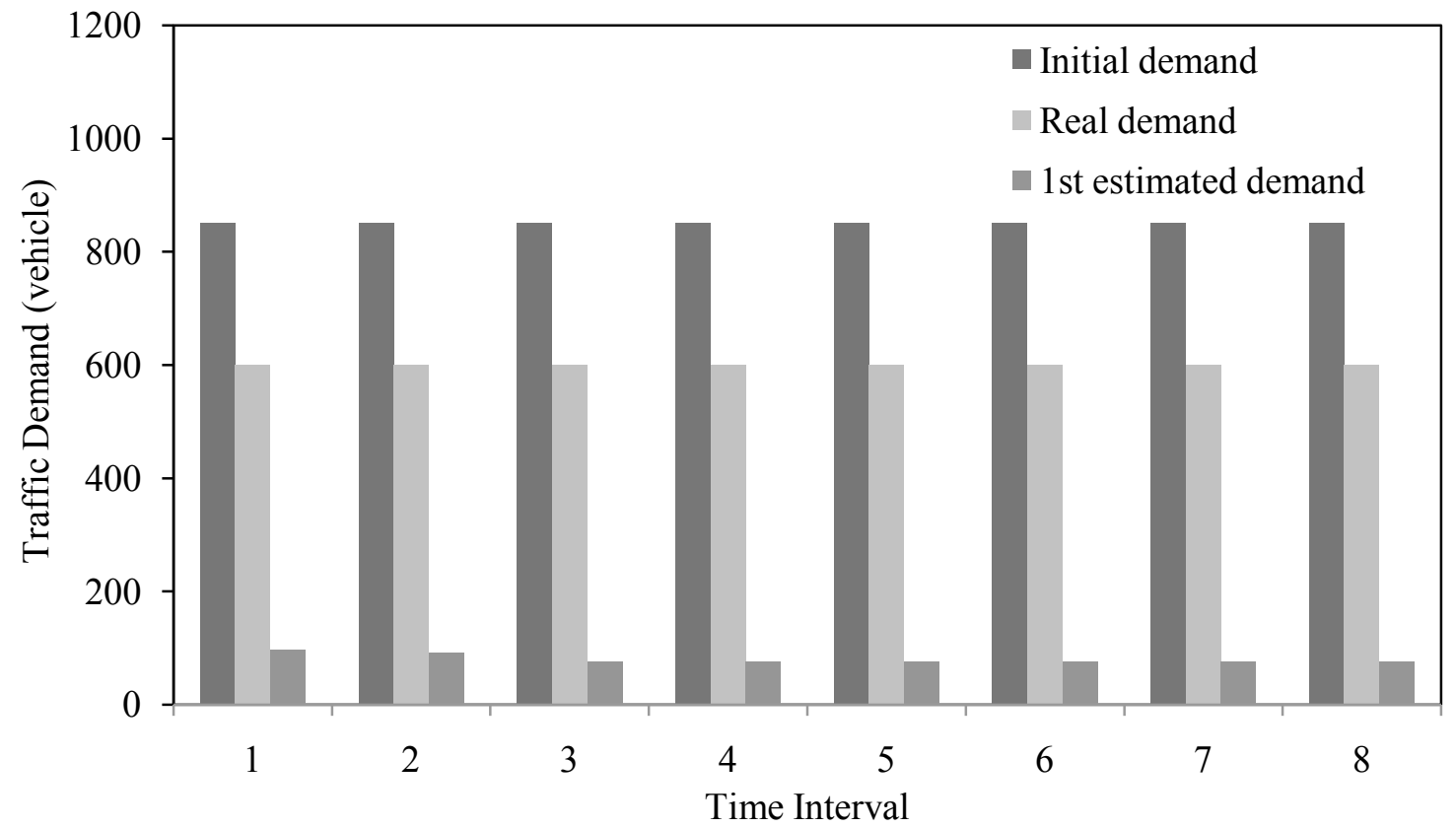

Figure 4.6 The comparison among real demand, initial demand and the estimated demand after the first iteration. 
Table 4.4 compares the real traffic demand to the estimated traffic demand. Based on the table, there is a small difference between the real and estimated dynamic O-D demand, and the maximum relative error is below $3 \%$.

Table 4.4 Real and estimated O-D demand.

\begin{tabular}{|l|l|l|l|l|l|l|l|l|}
\hline Time Intl. & 1 & 2 & 3 & 4 & 5 & 6 & 7 & 8 \\
\hline Real & 600 & 600 & 600 & 600 & 600 & 600 & 600 & 600 \\
\hline Estimated & 601 & 601 & 596 & 599 & 602 & 598 & 604 & 616 \\
\hline Difference & $0.17 \%$ & $0.17 \%$ & $-0.67 \%$ & $-0.17 \%$ & $0.33 \%$ & $-0.33 \%$ & $0.67 \%$ & $2.67 \%$ \\
\hline
\end{tabular}

Table 4.5 shows the comparison between real speed and assignment speed based on the estimated traffic demand. The comparison is illustrated in Figure 4.7. According to the table and the figure, one can see that the simulated traffic speed data, based on the estimated O-D matrix, are close to those based on the real O-D matrix. The maximum relative error between them is below $4 \%$.

Table 4.5 Real and estimated traffic speed.

\begin{tabular}{|l|l|l|l|l|l|l|l|l|}
\hline Time Intl. & 1 & 2 & 3 & 4 & 5 & 6 & 7 & 8 \\
\hline Real & 38.5 & 38.65 & 39.96 & 40.03 & 39.73 & 39.36 & 39.72 & 39.91 \\
\hline Estimated & 37.13 & 37.92 & 40.13 & 39.65 & 39.63 & 39.64 & 39.71 & 39.78 \\
\hline Difference & $-3.56 \%$ & $-1.89 \%$ & $0.43 \%$ & $-0.95 \%$ & $-0.25 \%$ & $0.71 \%$ & $-0.03 \%$ & $-0.33 \%$ \\
\hline
\end{tabular}

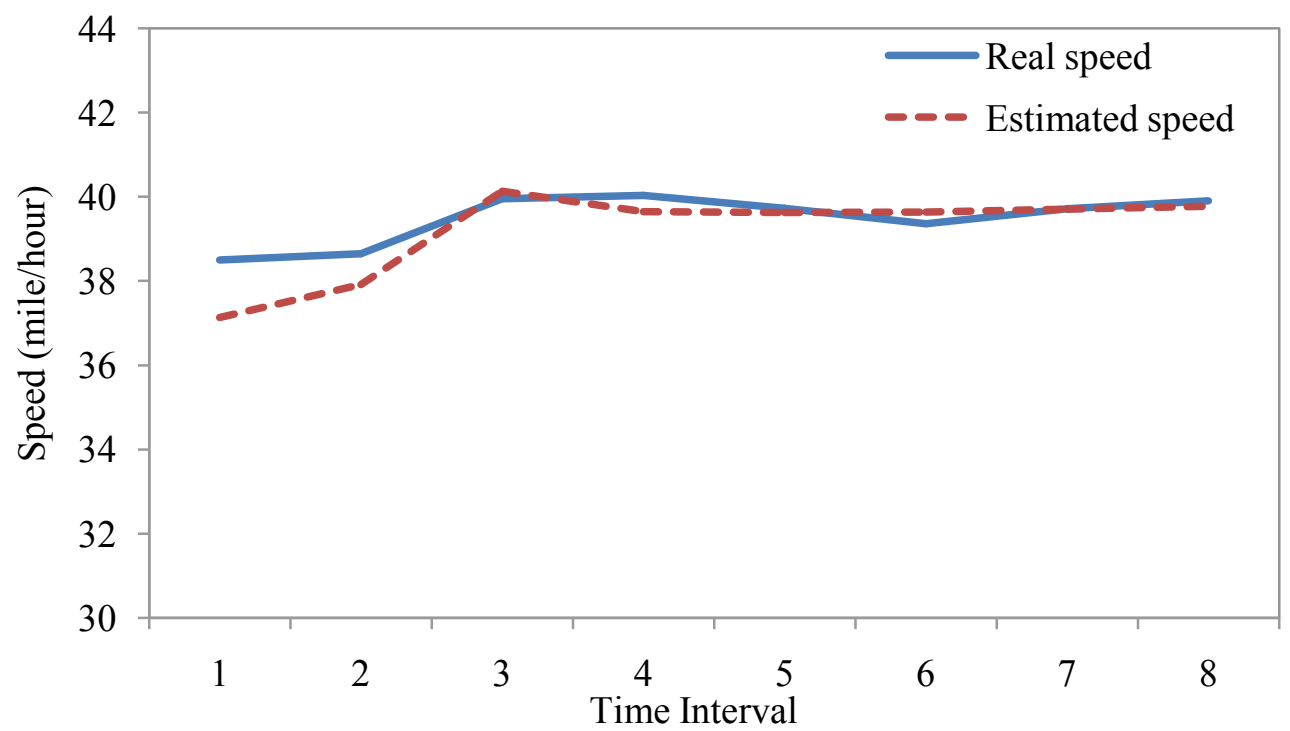

Figure 4.7 The comparison between real speed and estimated speed. 
Table 4.6 shows the comparison between real volume and assignment volume based on the estimated traffic demand. The comparison is illustrated in Figure 4.8. According to the table and figure, one can see that traffic volume data, based on the estimated O-D matrix, are close to those based on the real O-D matrix. The maximum relative error between them is below $4 \%$.

Table 4.6 Real and estimated traffic volume

\begin{tabular}{|l|l|l|l|l|l|l|l|l|}
\hline Time Intl. & 1 & 2 & 3 & 4 & 5 & 6 & 7 & 8 \\
\hline Real & 319 & 304 & 305 & 303 & 303 & 302 & 308 & 319 \\
\hline Estimated & 326 & 297 & 305 & 306 & 301 & 308 & 302 & 308 \\
\hline Difference & $2.19 \%$ & $-2.30 \%$ & $0.00 \%$ & $0.99 \%$ & $-0.66 \%$ & $1.99 \%$ & $-1.95 \%$ & $-3.45 \%$ \\
\hline
\end{tabular}

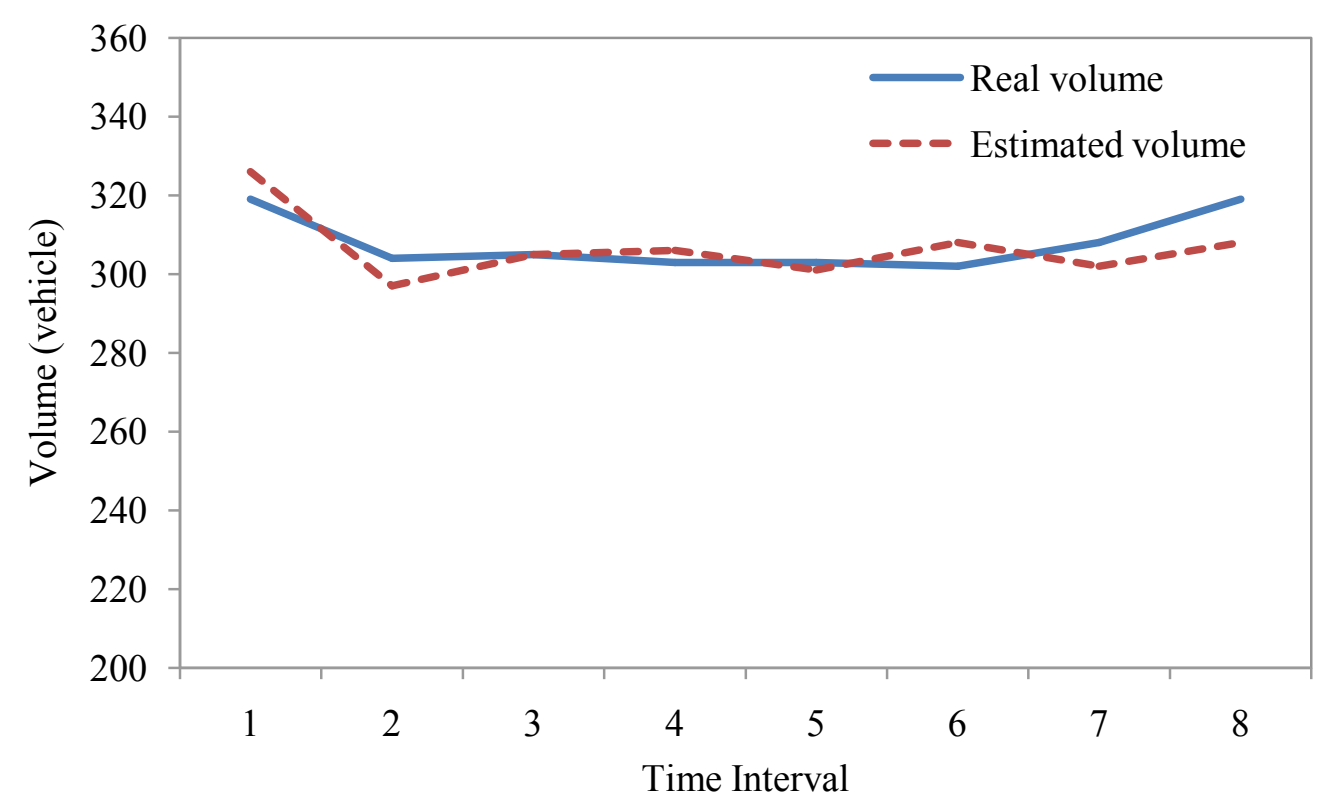

Figure 4.8 The comparison between real volume and estimated volume.

In order to demonstrate the benefits of the three terms in the proposed DODE, namely the adaptive weighting factor, the adaptive conversion factor, and the transition term, the estimation is performed using a before-and-after strategy. With this strategy, the performance of the model is tested for scenarios with and without the proposed terms.

Figure 4.9 shows the results of the before-and-after strategy. In the legend, "Proposed" stands for the estimation performance based on the proposed model. "WOW" 
represents the estimation performance based on the proposed model without the adaptive weighting factor term. "WOC" denotes the estimation performance based on the proposed model without the adaptive conversion factor term, and "WOT" represents the estimation performance based on the proposed model without the transition term. According to the figure, the performance of the estimation model is lower when excluding any of the three terms. The adaptive weighting factor seems to have the greatest contribution to the accuracy of the estimation result because without this adaptive factor, the objective function value does not decrease.

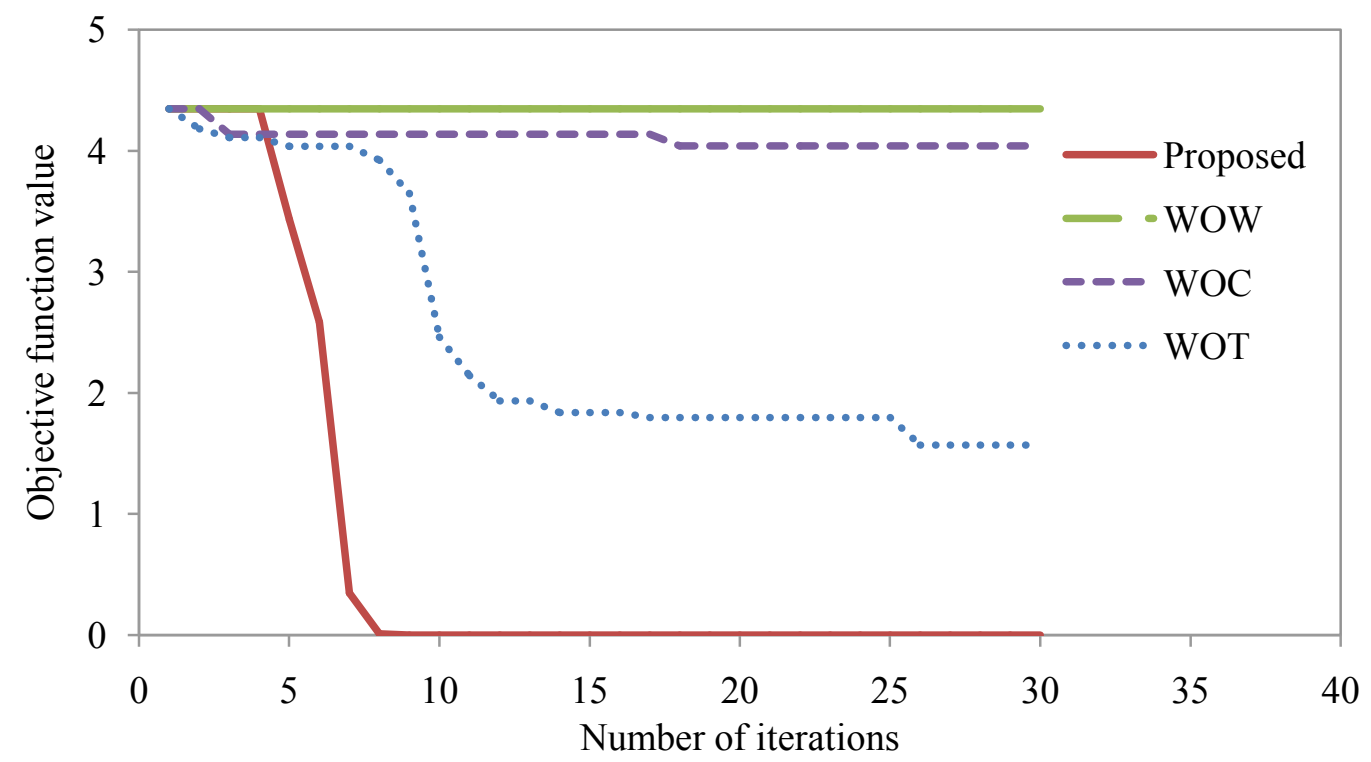

Figure 4.9 The comparison among the model performance before-and-after the proposed terms are employed.

In Table 4.7, the real O-D matrix is compared with the estimated O-D matrix. The relative error between the real and the estimated O-D matrix is represented by PROP_ERR (the relative error between the real O-D demand and the demand estimated by the proposed model), WOC_ERR (the relative error between the real O-D demand and the demand estimated by the model without an adaptive conversion factor term), 
WOT_ERR (the relative error between the real O-D demand and the demand estimated by the model without the transition factor term), and WOT_ERR (the relative error between the real O-D demand and the demand estimated by the model without the adaptive weighting factor term). Based on the table, one can see that the estimation results become poor without any of the proposed terms.

Table 4.7 The comparison of the estimation results before-and-after the proposed terms are used.

\begin{tabular}{|l|l|l|l|l|l|l|l|l|}
\hline Time Intl. & 1 & 2 & 3 & 4 & 5 & 6 & 7 & 8 \\
\hline Real & 600 & 600 & 600 & 600 & 600 & 600 & 600 & 600 \\
\hline Proposed & 601 & 601 & 596 & 599 & 602 & 598 & 604 & 616 \\
\hline WOC & 827 & 1862 & 1452 & 1234 & 1115 & 1047 & 1011 & 996 \\
\hline WOT & 543 & 703 & 0 & 0 & 745 & 554 & 617 & 713 \\
\hline WOW & 850 & 850 & 850 & 850 & 850 & 850 & 850 & 850 \\
\hline PROP_ERR & $0.17 \%$ & $0.17 \%$ & $-0.67 \%$ & $-0.17 \%$ & $0.33 \%$ & $-0.33 \%$ & $0.67 \%$ & $2.67 \%$ \\
\hline WOC_ERR & $38 \%$ & $210 \%$ & $142 \%$ & $106 \%$ & $86 \%$ & $75 \%$ & $69 \%$ & $66 \%$ \\
\hline WOT_ERR & $-10 \%$ & $17 \%$ & $-100 \%$ & $-100 \%$ & $24 \%$ & $-8 \%$ & $3 \%$ & $19 \%$ \\
\hline WOW_ERR & $42 \%$ & $42 \%$ & $42 \%$ & $42 \%$ & $42 \%$ & $42 \%$ & $42 \%$ & $42 \%$ \\
\hline
\end{tabular}

In order to demonstrate the advantages of the proposed model over the Zhou's model (without the proposed three terms), their performances are compared in Figure 4.10. According to the figure, Zhou's model does not even cause a decrease in the objective function. Table 4.8 shows the comparison between the estimation demands based on both the proposed and Zhou's estimation models, and also indicates the inability of Zhou's estimation model to deal with congested traffic conditions. 


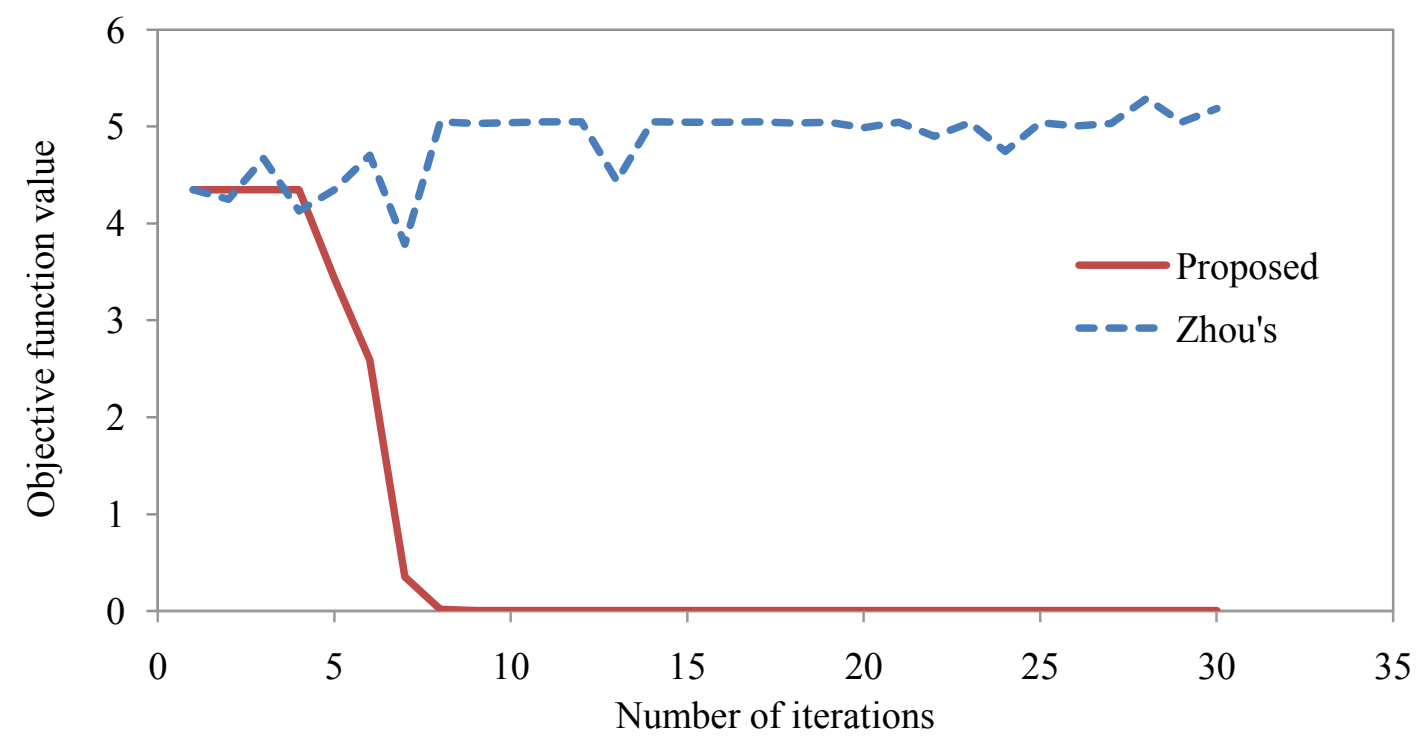

Figure 4.10 Comparison of performance between the proposed DODE model and the Zhou's model.

Table 4.8 compares the estimation results from the proposed DODE model with the results from Zhou's estimation model. In the table, PRO_ERR and TRD_ERR represent the relative errors of the proposed model and Zhou's model, respectively. According to the table, one can see that, for Zhou's model, the smallest estimation error is $12 \%$ and the largest error is $400 \%$. The main reason for this weakness in Zhou's model is that it holds the assumption of erroneous proportional assignment, and it cannot incorporate the useful observed speed data.

Table 4.8 The comparison of the estimation result based on the proposed DODE and Zhou's estimation model.

\begin{tabular}{|l|l|l|l|l|l|l|l|l|}
\hline Time Intl. & 1 & 2 & 3 & 4 & 5 & 6 & 7 & 8 \\
\hline Real & 600 & 600 & 600 & 600 & 600 & 600 & 600 & 600 \\
\hline Proposed & 601 & 601 & 596 & 599 & 602 & 598 & 604 & 616 \\
\hline Zhou's & 674 & 3000 & 851 & 845 & 849 & 855 & 850 & 852 \\
\hline PRO_ERR & $0.17 \%$ & $0.17 \%$ & $-0.67 \%$ & $-0.17 \%$ & $0.33 \%$ & $-0.33 \%$ & $0.67 \%$ & $2.67 \%$ \\
\hline TRD_ERR & $12 \%$ & $400 \%$ & $42 \%$ & $41 \%$ & $42 \%$ & $43 \%$ & $42 \%$ & $42 \%$ \\
\hline
\end{tabular}




\subsection{Summary}

In this chapter, the proposed dynamic O-D matrix estimation (DODE) model is implemented in a hypothetical road network. Using a before-and after-strategy, the three terms in the proposed model were proven effective. Finally, performance comparisons show that the proposed model demonstrates several advantages over Zhou's model.

It is worth mentioning that the better performance of the proposed model lies in three ideal conditions: (1) the links' traffic flow models are calibrated accurately; (2) the historical traffic demand pattern is known in advance; and (3) detectors installed on the road link can catch traffic information from all O-D pairs. In reality, these conditions are not easy to meet. Nevertheless, the proposed estimation model shows an overall robust performance, as will be demonstrated by the case studies in the following two chapters. 


\section{CHAPTER 5}

\section{A CASE STUDY IN I-95}

This chapter aims to demonstrate the feasibility and strength of the proposed methodology for a midsized road network. In this chapter, Section 5.1 discusses preparing the road network. Traffic flow models are calibrated in Section 5.2. Initial O-D matrix is estimated in Sections 5.3 and 5.4. Dynamic O-D matrix is estimated in Section 5.5. Traffic flow models are fine-tuned in Section 5.6. Finally, the dynamic O-D matrix is re-estimated based on the fine-tuned traffic flow model in Section 5.7.

\subsection{Road Network Configuration}

The study road network is a section of northbound interstate highway I-95 in MiamiDade County, Florida. The road segment under study is between NW 62 Street and NW 183 Street. The CUBE is used to extract the study road network and initial traffic demand matrix from the Southeast Florida Regional Planning Model (SERPM 6.5). The extracted road network (Figure 5.1) comprises 68 links and 21 zones. In Figure 5.1, the links with shallow color indicate that detectors are installed. In the boxes of the figure are the zone numbers. The study time horizon is the afternoon peak-period (three hours) on a typical work day. The basic time interval for the dynamic O-D matrix is set at 15 minutes, since that duration is considered adequate for a traveler to traverse a given section of the road network. The number of the basic time intervals is $3 \times 60 / 15=12$ for the dynamic O-D matrix. Initially, the number of unknown O-D demand variables is $21 \times 21 \times 12$.

Traffic detectors were installed by the Florida Department of Transportation (FDOT) District 6. Traffic data including traffic volume traffic speed and traffic occupancy are collected by these detectors every 20 seconds. In the study road network, 
there are a total of 18 detectors, and 10 of them are installed on the main stream of I-95. The other eight detectors are installed in the at-ramp of I-95, and they only collect traffic volume data. The collected traffic measurement data are aggregated into 15-minute intervals.

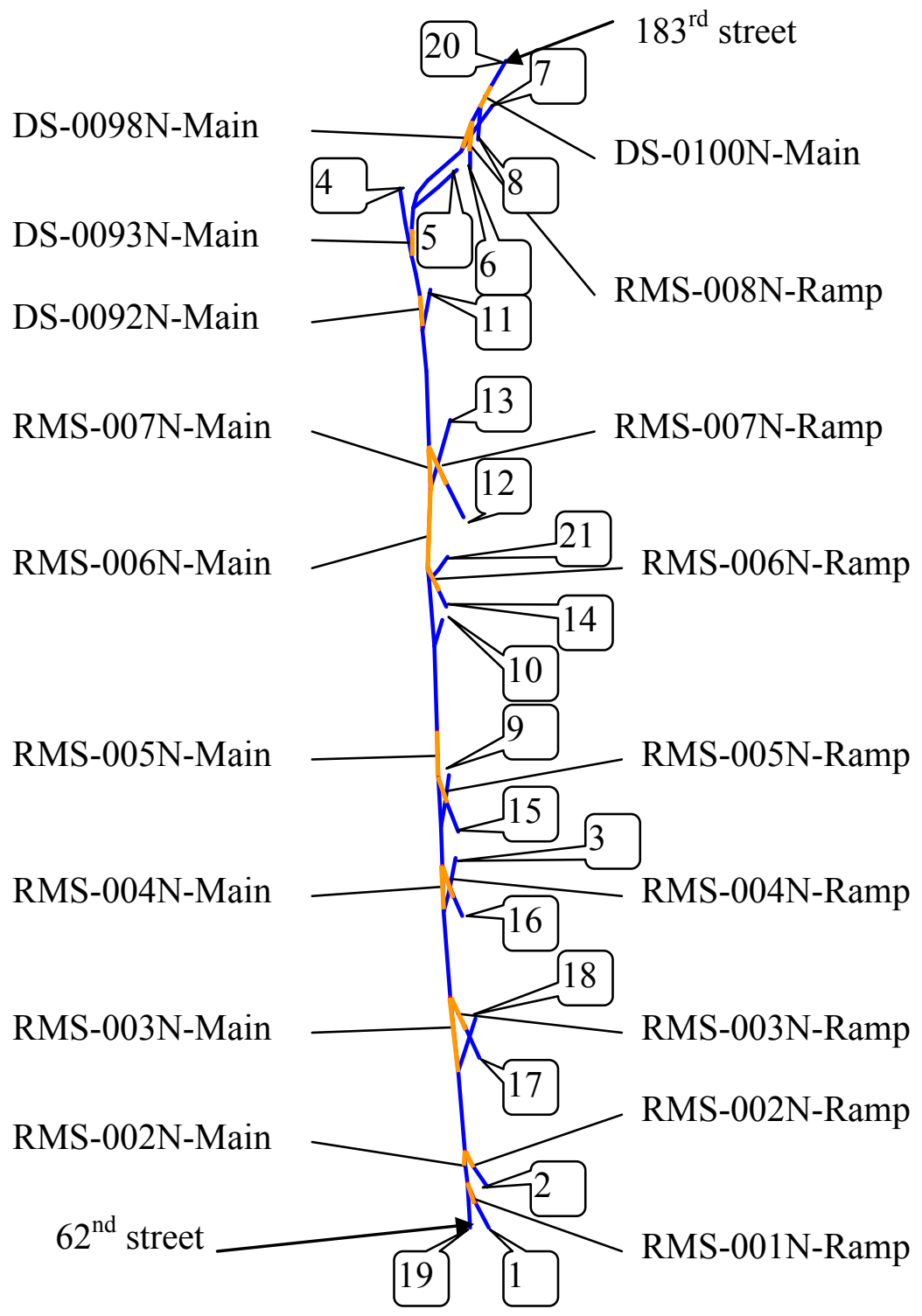

Figure 5.1 Road network structure and detector locations. 


\subsection{Traffic Flow Model Calibration}

Based on the proposed linear regression algorithm, traffic flow models can be easily calibrated. There are 10 links in the network with detectors collecting both traffic speed and traffic occupancy data. Traffic occupancy data can be roughly converted into density data based on Equation 5.1, given the average length of vehicles and detectors.

$$
D=\frac{5,280 \times O}{L_{v}+L_{d}}
$$

where $O$ is the occupancy, $D$ is the traffic density data (veh/mi/ln), $L_{v}$ is the average length of vehicles (in feet), and $L_{d}$ is the length of the detector (in feet).

With the available traffic data, the corresponding 10 traffic flow models can be calibrated as shown in Table 5.1. In the table, parameters $\alpha, U_{f}, K_{j}$, and $K_{b}$ are selected for later fine-tuning and their range of values, obtained from the scatter plot, are also provided as the values included in brackets. $V_{f}$ is dependent on other variables based on the traffic flow model. According to the speed and density plot, there is not much information on $K_{j}$, thus the values of $K_{j}$ and $V_{f}$ are fixed .

Table 5.1 Results of traffic flow model calibration.

\begin{tabular}{|c|c|c|c|r|c|c|c|}
\hline model \# & $\alpha$ & $U_{f}$ & $V_{0}$ & $V_{f}$ & $K_{j}$ & $K_{b}$ & $\mathrm{R}^{2}$ \\
\hline 1 & $5.30[1.2,10]$ & $60[55,60]$ & $12[8,15]$ & 109 & 200 & $25[10,35]$ & 0.70 \\
\hline 2 & $5.63[1.2,10]$ & $59[54,64]$ & $12[8,15]$ & 112 & 200 & $25[15,35]$ & 0.60 \\
\hline 3 & $3.38[1.2,10]$ & $55[50,60]$ & $12[8,15]$ & 80 & 200 & $25[15,35]$ & 0.70 \\
\hline 4 & $4.14[1.2,10]$ & $66[61,71]$ & $12[8,15]$ & 106 & 200 & $25[15,35]$ & 0.73 \\
\hline 5 & $4.47[1.2,10]$ & $60[55,65]$ & $12[8,15]$ & 99 & 200 & $25[15,35]$ & 0.58 \\
\hline 6 & $4.15[1.2,10]$ & $63[58,68]$ & $12[8,15]$ & 101 & 200 & $25[15,35]$ & 0.58 \\
\hline 7 & $4.25[1.2,10]$ & $59[54,64]$ & $12[8,15]$ & 95 & 200 & $25[15,35]$ & 0.57 \\
\hline 8 & $3.96[1.2,10]$ & $60[55,65]$ & $12[8,15]$ & 93 & 200 & $25[15,35]$ & 0.51 \\
\hline 9 & $4.63[1.2,10]$ & $63[58,68]$ & $12[8,15]$ & 107 & 200 & $25[15,35]$ & 0.52 \\
\hline 10 & $3.00[1.2,10]$ & $56[51,61]$ & $12[8,15]$ & 78 & 200 & $25[15,35]$ & 0.76 \\
\hline
\end{tabular}


Figure 5.2 shows the calibration result for a typical traffic flow model, which depicts the general density-speed relationship for a road link.

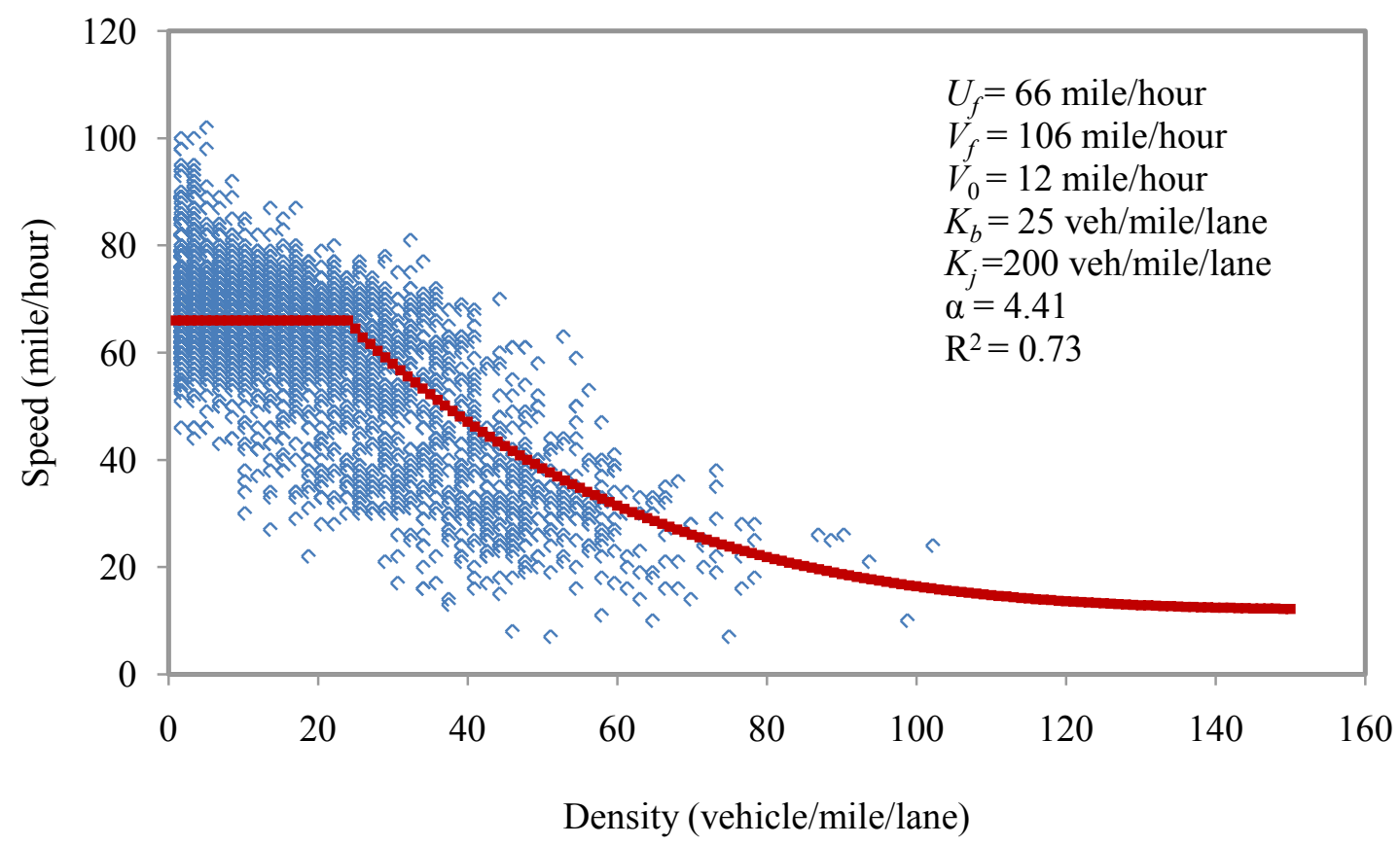

Figure 5.2 A typical traffic flow model calibration.

There are a total of 32 mainstream links in the study network. The 10 traffic flow models are simply assigned to these links in accordance with the distance between these links and the detectors. For ramps, because speed and occupancy data are unavailable, the default traffic flow model functions in the DYNASMART-P are used for them, which will not be adjusted in the traffic flow model fine-tuning (TFMFT) step.

\subsection{Static Initial O-D Matrix Estimation (SIODE)}

An O-D matrix can be extracted from the SERPM model for an afternoon peak-period of three hours. It is then factorized into 15-minute, sub-period O-D matrices based on the traffic volume distribution pattern. These factorized O-D matrices are subsequently calibrated based on the 15-minute observed traffic volume data by using the proposed initial O-D matrix estimation (SIODE) model. 
The SIODE model can be implemented by CUBE ANALYST (Citilabs 2004). CUBE ANALYST is a module of the CUBE developed specifically for estimating trip matrices based on the maximum likelihood model. CUBE ANALYST enables the user to exploit a wide variety of data to estimate the O-D matrix. The flow chart of SIODE implemented by CUBE ANALYST is presented in Figure 5.3.

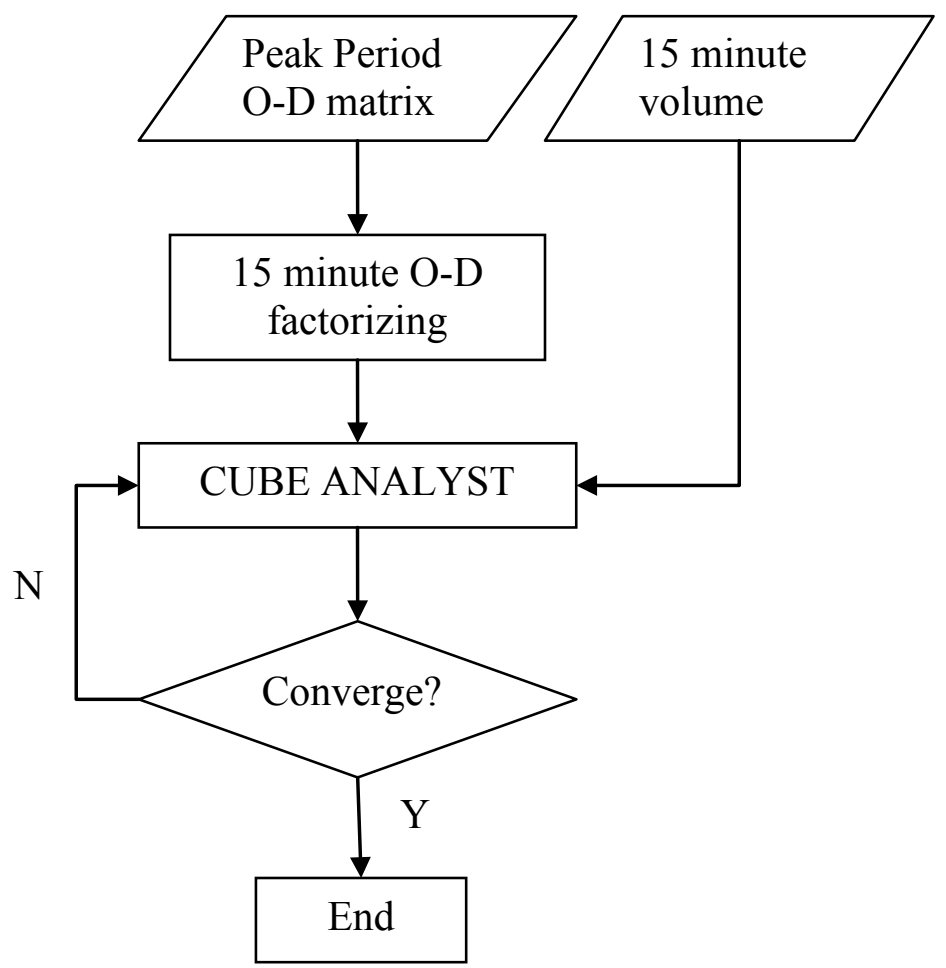

Figure 5.3 Flowchart of static initial O-D matrix estimation (SIODE).

The estimation result is evaluated based on the comparison between the static assignment traffic volumes with the O-D matrix before-and-after SIODE. RMSPE (root mean square percent error) is chosen to evaluate the estimation results, as shown:

$$
R M S P E=\sqrt{\frac{1}{N} \sum_{n=1}^{N}\left[\frac{Y_{n}^{s}-Y_{n}^{o}}{Y_{n}^{o}}\right]^{2}}
$$


where $Y_{n}^{S}$ is the assignment item for $n$ time intervals and $Y_{n}^{o}$ is the observed item for $n$ time intervals. In this case study, $n=12$.

The final results are shown in Table 5.2. Based on the last two columns of the table, it can be seen that after SIODE, there is a significant improvement in RMSPE between observed traffic volume and assigned traffic volume for screenlines. The average of the RMSPE for volume has been reduced from 0.32 to 0.07 .

Table 5.2 Comparison between static assignment traffic volumes before-and-after SIODE.

\begin{tabular}{|c|c|c|c|c|c|}
\hline \multirow{2}{*}{$\begin{array}{c}\text { Screen } \\
\text { line }\end{array}$} & \multirow{2}{*}{$\begin{array}{c}\text { Avg. } \\
\text { observed } \\
\text { volume per } \\
15 \text { minutes }\end{array}$} & \multicolumn{2}{|c|}{$\begin{array}{l}\text { Avg. assignment volume per } \\
15 \text { minutes }\end{array}$} & \multicolumn{2}{|c|}{ RMSPE for volume } \\
\hline & & Before SIODE & After SIODE & $\begin{array}{l}\text { Before } \\
\text { SIODE }\end{array}$ & $\begin{array}{l}\text { After } \\
\text { SIODE }\end{array}$ \\
\hline 1 & 1,148 & 867 & 1,193 & 0.24 & 0.04 \\
\hline 2 & 798 & 577 & 866 & 0.28 & 0.09 \\
\hline 3 & 1,376 & 899 & 1,234 & 0.35 & 0.10 \\
\hline 4 & 2,202 & 1,532 & 2,128 & 0.30 & 0.04 \\
\hline 5 & 2,133 & 1,457 & 1,989 & 0.32 & 0.07 \\
\hline 6 & 2,129 & 1,626 & 2,129 & 0.24 & 0.01 \\
\hline 7 & 1,869 & 1,683 & 2,068 & 0.10 & 0.11 \\
\hline 8 & 2,039 & 1,580 & 1,999 & 0.23 & 0.02 \\
\hline 9 & 2,066 & 1,640 & 1,991 & 0.21 & 0.04 \\
\hline 10 & 2,081 & 1,676 & 2,041 & 0.20 & 0.02 \\
\hline 11 & 174 & 63 & 158 & 0.64 & 0.09 \\
\hline 12 & 59 & 81 & 63 & 0.41 & 0.08 \\
\hline 13 & 180 & 66 & 157 & 0.63 & 0.13 \\
\hline 14 & 98 & 118 & 98 & 0.24 & 0.02 \\
\hline 15 & 138 & 100 & 126 & 0.27 & 0.08 \\
\hline 16 & 192 & 88 & 181 & 0.54 & 0.06 \\
\hline 17 & 188 & 101 & 170 & 0.46 & 0.10 \\
\hline 18 & 181 & 201 & 195 & 0.14 & 0.08 \\
\hline Avg & 1,058 & 798 & 1,044 & 0.32 & 0.07 \\
\hline
\end{tabular}

Figure 5.4 illustrates the comparison of RMSPE for volumes before-and-after SIODE. According to the figure, it can be seen that the RMSPE for volume has been reduced at almost every detector after SIODE. 


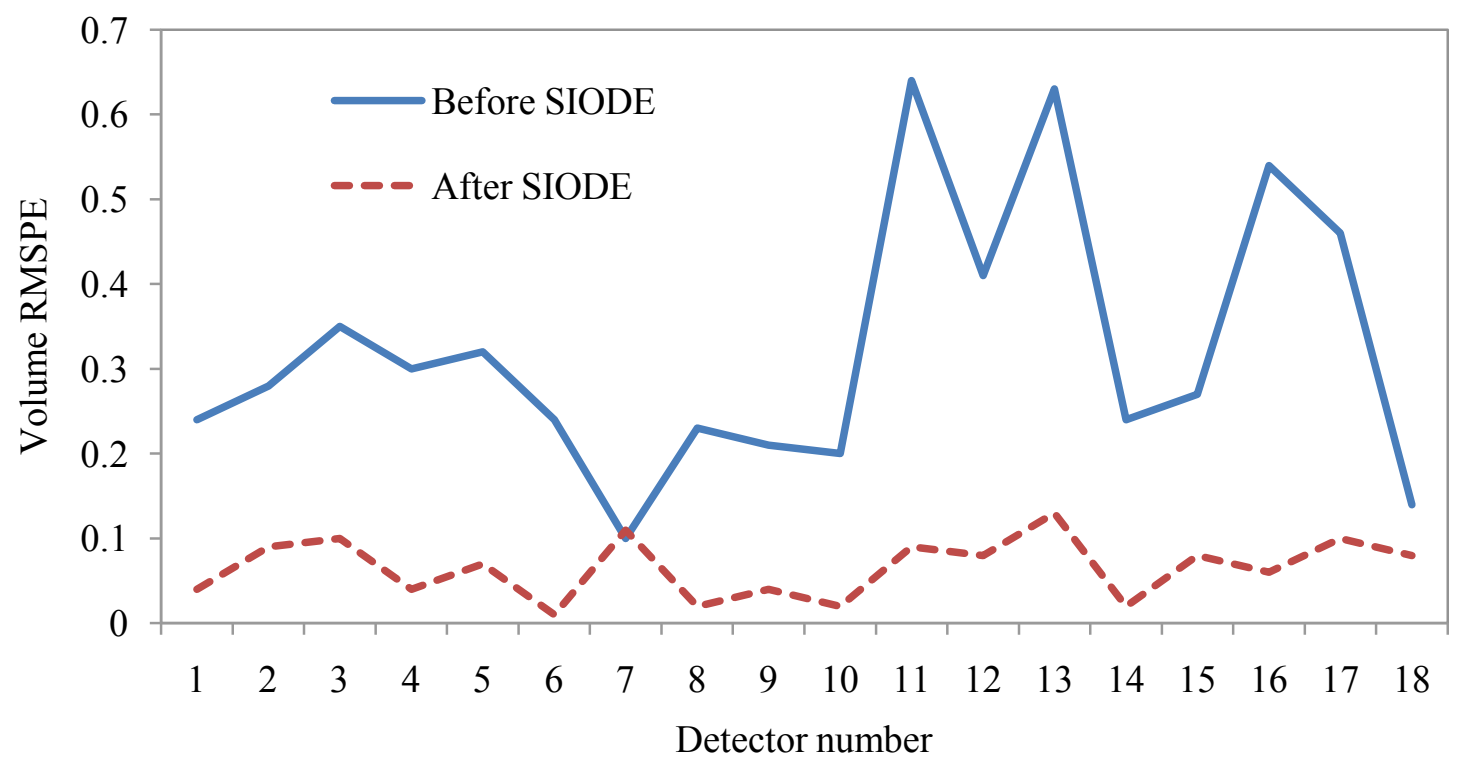

Figure 5.4 Comparison of assignment volume RMSPE before and after SIODE

\subsection{Dynamic Initial O-D Matrix Estimation (DIODE)}

The DIODE model is used to improve the result of SIODE with the aid of dynamic traffic assignment. The two sub-models in DIODE are employed, and their performance is illustrated by Figures 5.5 and 5.6, respectively. Sub-model 1 reaches convergence after one iteration, and the objective function value drops from 1.395 to 1.372 . The Sub-model 2 reaches convergence after two iterations, and the objective function value drops from 1.372 to 1.331. Although Equation 3.11 is used to estimate the dynamic O-D matrix, due to the effect of the adaptive weighting factor, the adaptive conversion factor, and the transition term, the value of Equation 3.11 cannot reflect the real deviation between the simulated and observed traffic volumes. In those two Figures, the objective function value is calculated based on the sum square of relative errors (SSRE) of observed volumes and simulated volumes, which are defined as SSRE $=\sum_{k, t}\left[\left(V_{k, t}-V_{k}^{O}, t\right) / V_{k}^{O},\right]^{2}$. 
DIODE does not significantly reduce the objective function in this case study. This is because the network size is small and there is not much difference between the static and dynamic route choices that SIODE has already given a good initial O-D matrix estimation. When a road network becomes large, DIODE can significantly improve the initial estimation result of the SIODE, which will be shown in the next chapter. It should be mentioned that the DIODE models have advantages in saving computation time, for they involve only a few variables. In this case study, Sub-model 1 has one variable, and the Sub-model 2 contains 12 variables. With the aid of the DIODE models, the estimation results are further improved, which is shown in Figure 5.7.

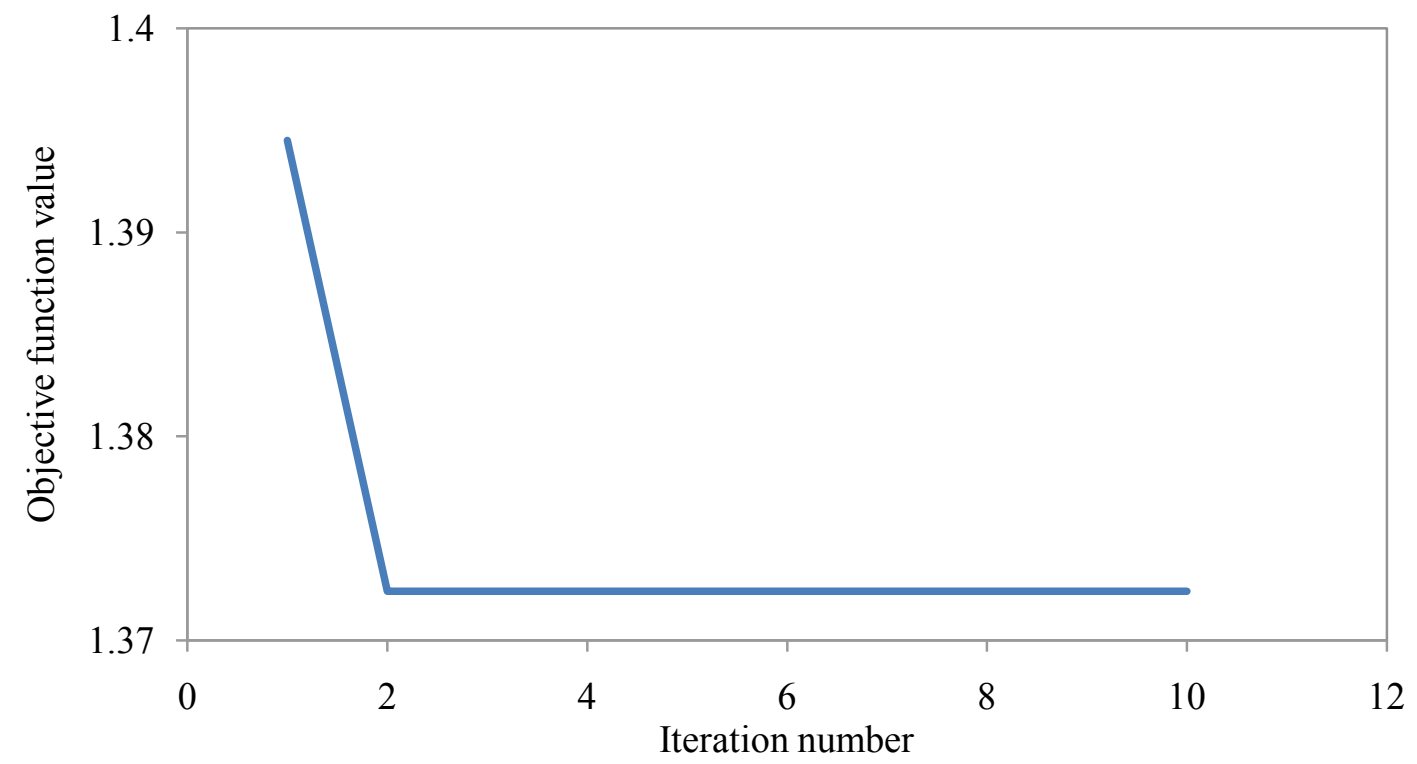

Figure 5.5 Performance of DIODE model 1. 


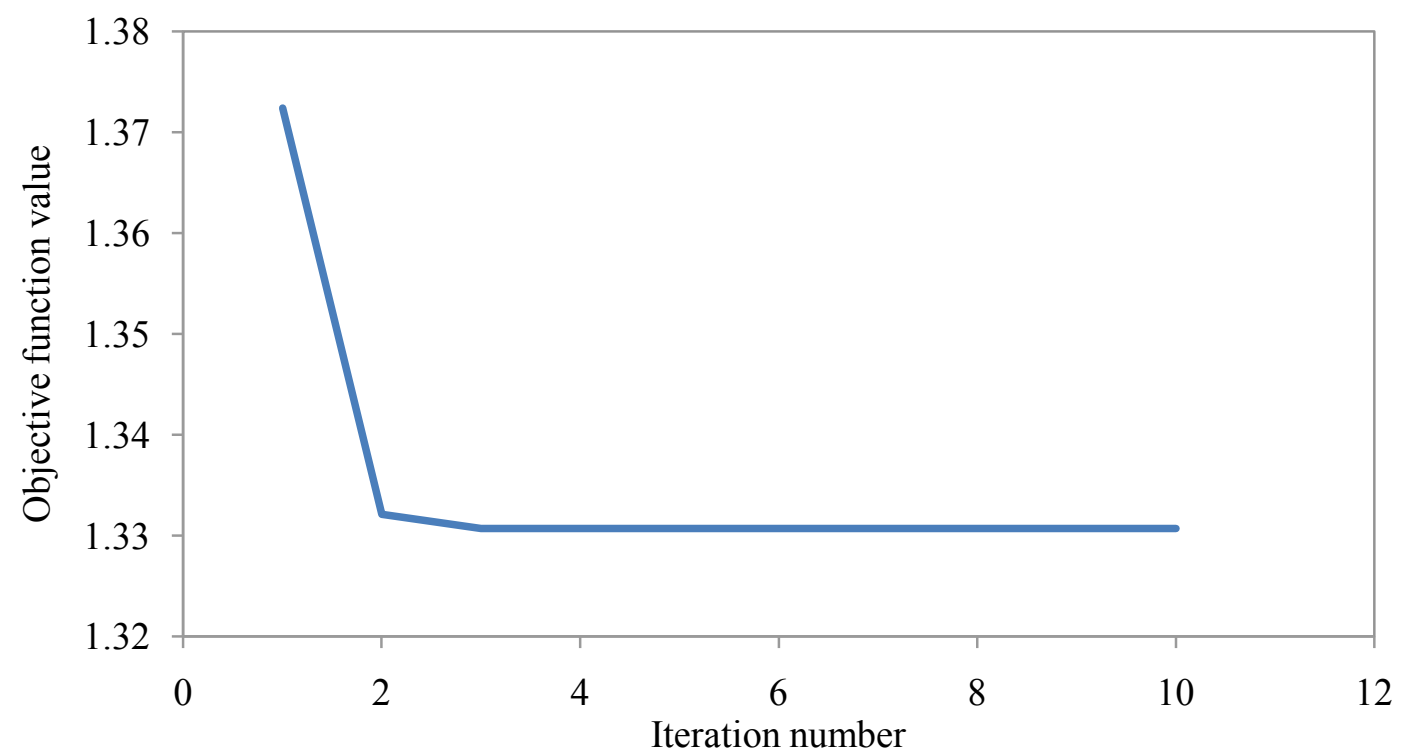

Figure 5.6 Performance of DIODE model 2.

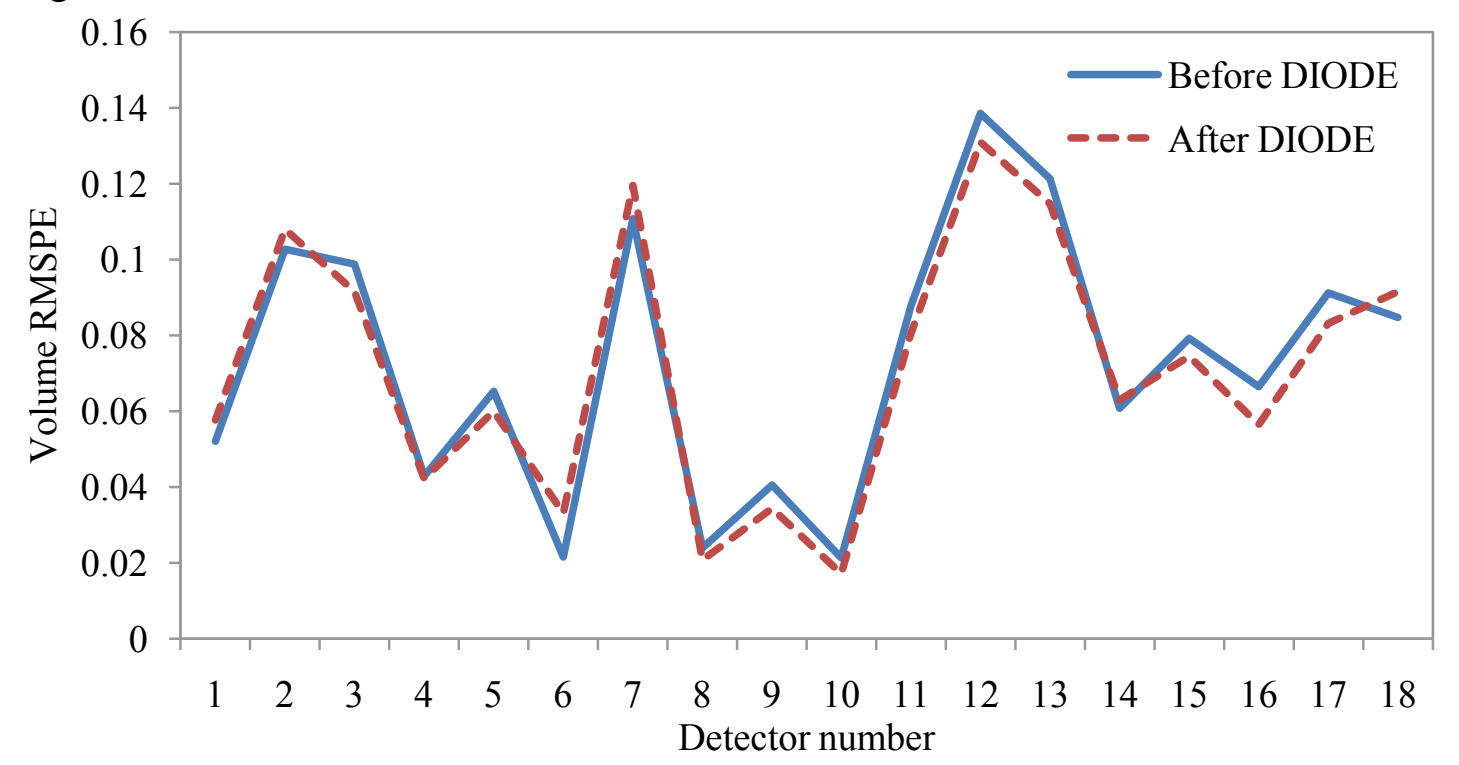

Figure 5.7 Comparison of RMSPE for assignment volume before-and-after DIODE.

In Figure 5.7, the solid line represents the RMSPE between the observed volume and the dynamic assignment volume based on the O-D demand estimated by the SIODE model. The dashed line stands for the RMSPE between the observed volume and the dynamic assignment volume based on the O-D demand estimated by the DIODE. The detailed traffic volume deviation before-and-after DIODE is illustrated in Table 5.3. 
Based on the table, the average RMSPE for volume has been reduced from 0.73 to 0.71 after DIODE.

Table 5.3 Comparison of traffic volume RMSPE before-and-after DIODE.

\begin{tabular}{|c|c|c|c|c|c|}
\hline \multirow{2}{*}{$\begin{array}{l}\text { Screen } \\
\text { line }\end{array}$} & \multirow{2}{*}{$\begin{array}{l}\text { Avg. } \\
\text { observed } \\
\text { volume per } \\
15 \text { minutes }\end{array}$} & \multicolumn{2}{|c|}{$\begin{array}{l}\text { Avg. assignment volume per } \\
15 \text { minutes }\end{array}$} & \multicolumn{2}{|c|}{ RMSPE for volume } \\
\hline & & $\begin{array}{l}\text { Before } \\
\text { DIODE }\end{array}$ & $\begin{array}{l}\text { After } \\
\text { DIODE }\end{array}$ & $\begin{array}{l}\text { Before } \\
\text { DIODE }\end{array}$ & $\begin{array}{l}\text { After } \\
\text { DIODE }\end{array}$ \\
\hline 1 & 1,148 & 1199 & 1208 & 0.052 & 0.058 \\
\hline 2 & 798 & 871 & 877 & 0.103 & 0.108 \\
\hline 3 & 1,376 & 1241 & 1250 & 0.099 & 0.092 \\
\hline 4 & 2,202 & 2142 & 2159 & 0.043 & 0.042 \\
\hline 5 & 2,133 & 2001 & 2016 & 0.065 & 0.060 \\
\hline 6 & 2,129 & 2140 & 2157 & 0.022 & 0.033 \\
\hline 7 & 1,869 & 2075 & 2091 & 0.111 & 0.120 \\
\hline 8 & 2,039 & 2000 & 2017 & 0.024 & 0.021 \\
\hline 9 & 2,066 & 1987 & 2003 & 0.041 & 0.034 \\
\hline 10 & 2,081 & 2042 & 2060 & 0.021 & 0.017 \\
\hline 11 & 174 & 159 & 160 & 0.088 & 0.080 \\
\hline 12 & 59 & 63 & 63 & 0.139 & 0.131 \\
\hline 13 & 180 & 158 & 160 & 0.121 & 0.115 \\
\hline 14 & 98 & 101 & 102 & 0.061 & 0.063 \\
\hline 15 & 138 & 127 & 128 & 0.079 & 0.075 \\
\hline 16 & 192 & 181 & 182 & 0.067 & 0.057 \\
\hline 17 & 188 & 172 & 173 & 0.091 & 0.083 \\
\hline 18 & 181 & 195 & 197 & 0.085 & 0.092 \\
\hline Avg. & 1,058 & 1,047 & 1,056 & 0.073 & 0.071 \\
\hline
\end{tabular}

\subsection{Dynamic O-D Matrix Estimation (DODE)}

With the estimated initial O-D matrix as demand side input and the calibrated traffic flow models as supply side input, the proposed dynamic O-D matrix estimation (DODE) model is used to perform the dynamic O-D matrix estimation. Nonlinear integer programming is used to solve the upper-level problem, and DYNASMART-P is used to solve the dynamic traffic loading problem in the lower-level. It takes four iterations for the DODE model to reach a convergent solution, as illustrated in Figure 5.8. The objective function value is reduced from 1.33 to 0.025 . 


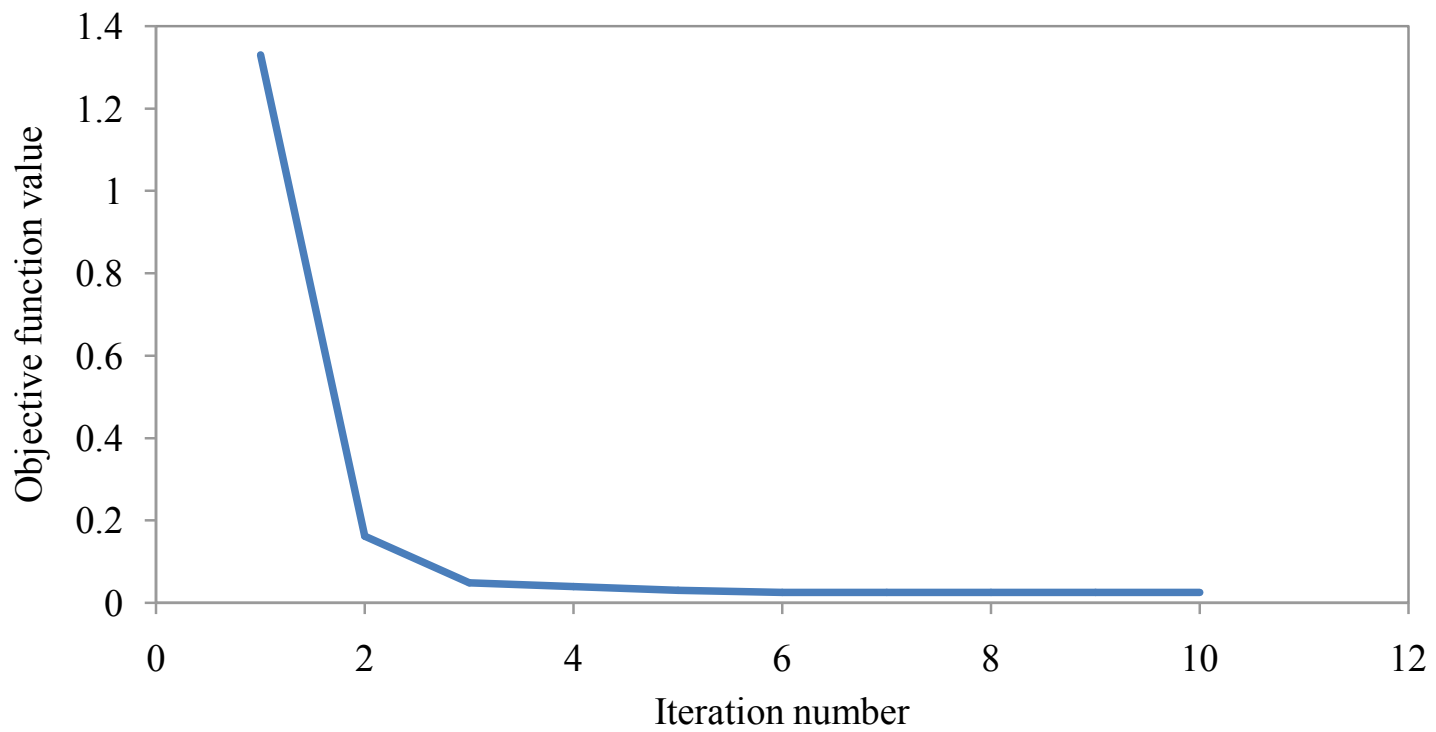

Figure 5.8 Performance of the DODE model.

Tables 5.4 and 5.5 provide a comparison of traffic volume and speed, respectively, after assigning the estimated dynamic O-D matrix onto the road network. According to Table 5.4, it can be seen that after the derived DODE simulated volumes are close to the observed volumes, the average RMSPE for volume is reduced from 0.07 to 0.01 . The comparison in Table 5.4 is illustrated in Figure 5.9. However, there are still large deviations in the RMSPE for speed after the DODE, according to Table 5.5. In order to reduce such differences, the traffic flow model fine-tuning (TFMFT) is performed, as is presented in the next section. 


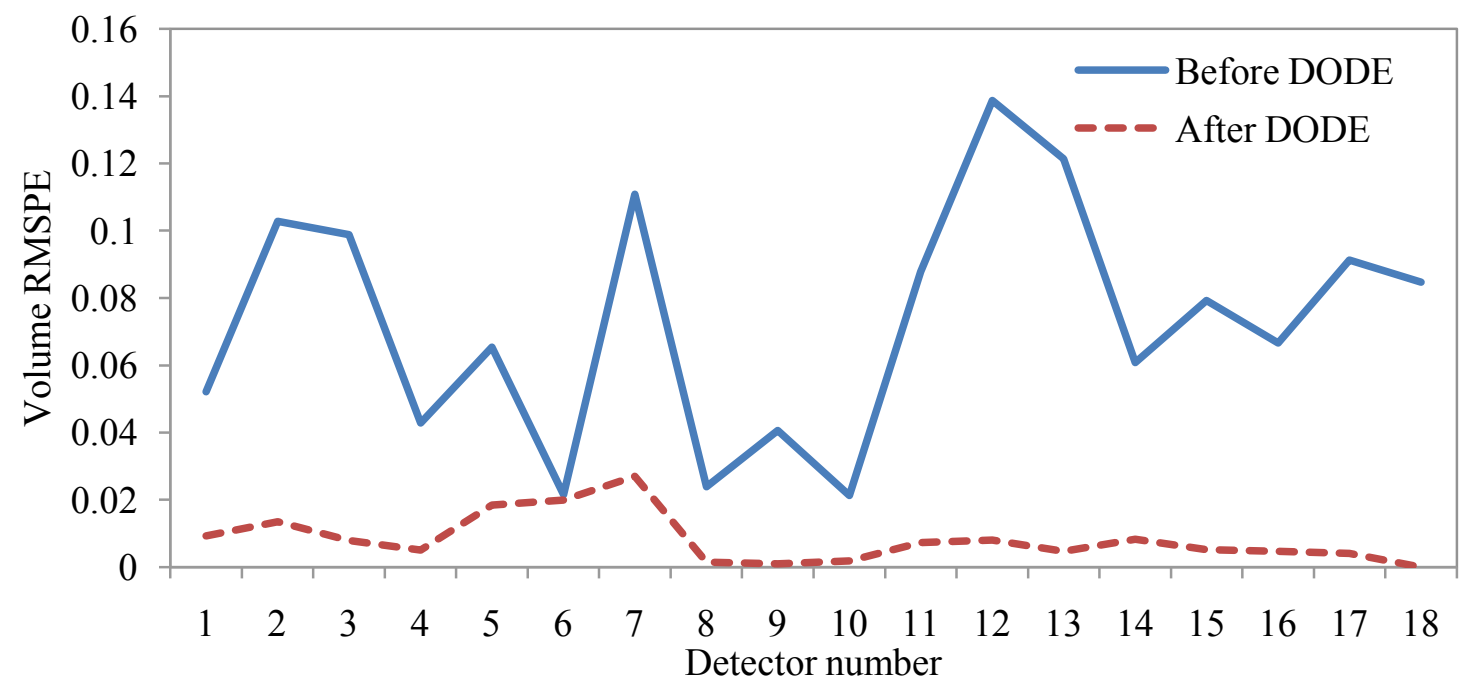

Figure 5.9 Comparison of RMSPE for assignment volume before and after DODE.

Table 5.4 Comparison of assignment traffic volume before-and-after DODE.

\begin{tabular}{|c|r|r|r|r|r|}
\hline \multirow{2}{*}{$\begin{array}{l}\text { Screen } \\
\text { Line }\end{array}$} & \multirow{2}{*}{$\begin{array}{l}\text { Avg. Observed } \\
\text { Volume per } 15\end{array}$} & \multicolumn{2}{l|}{$\begin{array}{l}\text { Avg. Simulated volume per } \\
\text { 15 Minutes }\end{array}$} & \multicolumn{2}{l|}{ RMSPE for Volume } \\
\cline { 3 - 6 } & Minutes & Before DODE & After DODE & Before DODE & After DODE \\
\hline 1 & 1,148 & 1,208 & 1,161 & 0.058 & 0.009 \\
\hline 2 & 798 & 877 & 794 & 0.108 & 0.013 \\
\hline 3 & 1,376 & 1,250 & 1,373 & 0.092 & 0.008 \\
\hline 4 & 2,202 & 2,159 & 2,186 & 0.042 & 0.005 \\
\hline 5 & 2,133 & 2,016 & 2,035 & 0.060 & 0.018 \\
\hline 6 & 2,129 & 2,157 & 2,103 & 0.033 & 0.012 \\
\hline 7 & 1,869 & 2,091 & 1,972 & 0.120 & 0.027 \\
\hline 8 & 2,039 & 2,017 & 2,027 & 0.021 & 0.002 \\
\hline 9 & 2,066 & 2,003 & 2,049 & 0.034 & 0.001 \\
\hline 10 & 2,081 & 2,060 & 2,102 & 0.017 & 0.002 \\
\hline 11 & 174 & 160 & 174 & 0.080 & 0.007 \\
\hline 12 & 59 & 63 & 59 & 0.131 & 0.008 \\
\hline 13 & 180 & 160 & 180 & 0.115 & 0.005 \\
\hline 14 & 98 & 102 & 98 & 0.063 & 0.008 \\
\hline 15 & 138 & 128 & 137 & 0.075 & 0.005 \\
\hline 16 & 192 & 182 & 193 & 0.057 & 0.005 \\
\hline 17 & 188 & 173 & 188 & 0.083 & 0.004 \\
\hline 18 & 181 & 197 & 181 & 0.092 & 0 \\
\hline Avg. & 1,058 & 1,056 & 1,056 & 0.07 & 0.01 \\
\hline
\end{tabular}


Table 5.5 Comparison of traffic speed before and after DODE.

\begin{tabular}{|c|c|c|c|c|c|}
\hline \multirow{2}{*}{$\begin{array}{l}\text { Screen } \\
\text { Line }\end{array}$} & \multirow{2}{*}{$\begin{array}{l}\text { Avg. } \\
\text { Observed } \\
\text { Speed per } \\
15 \text { Minutes }\end{array}$} & \multicolumn{2}{|c|}{$\begin{array}{l}\text { Avg. Simulated Speed per } 15 \\
\text { Minutes }\end{array}$} & \multicolumn{2}{|c|}{ RMSPE for Speed } \\
\hline & & Before DODE & After DODE & Before DODE & After DODE \\
\hline 1 & 45 & 49 & 50 & 0.169 & 0.181 \\
\hline 2 & 55 & 58 & 58 & 0.068 & 0.068 \\
\hline 3 & 45 & 53 & 51 & 0.212 & 0.181 \\
\hline 4 & 43 & 59 & 55 & 0.549 & 0.522 \\
\hline 5 & 48 & 62 & 61 & 0.416 & 0.406 \\
\hline 6 & 51 & 60 & 60 & 0.199 & 0.194 \\
\hline 7 & 42 & 59 & 58 & 0.412 & 0.403 \\
\hline 8 & 48 & 62 & 62 & 0.310 & 0.304 \\
\hline 9 & 44 & 59 & 58 & 0.350 & 0.331 \\
\hline 10 & 53 & 66 & 65 & 0.256 & 0.239 \\
\hline Avg. & 47 & 59 & 58 & 0.294 & 0.283 \\
\hline
\end{tabular}

For the purpose of comparison, Zhou's dynamic O-D matrix estimation model is used to perform the estimation in this case study. The initial O-D matrix is extracted from the SERPM model and factorized into multi-period O-D matrices by the volume proportional factor. The traffic flow models are used from Table 5.1. The performance of Zhou's estimation model is shown in Figure 5.10. According to the figure, the objective function value drops from 28.06 to 0.25 after 50 iterations. In the proposed methodology framework, with negligible computation time spent in SIODE, it takes three iterations for the DIODE model to reduce the objective function value from 28.06 to 1.33 . Finally, it takes four iterations for the DODE to reach the final objective function value of 0.025 . The total number of iterations for the proposed methodology framework is seven, and the final objective function value is 0.025 . The comparison of the traffic volume and speed between the proposed model and Zhou's model is presented in Table 5.6. One can see from the table that after Zhou's model estimation, the average RMSPE for volume is 0.023 and for speed 0.285 . After DODE, the average RMSPE for volume is 0.010 and for 
speed 0.283 . The conclusion is that the proposed methodology framework performs better than Zhou's model in this case study.

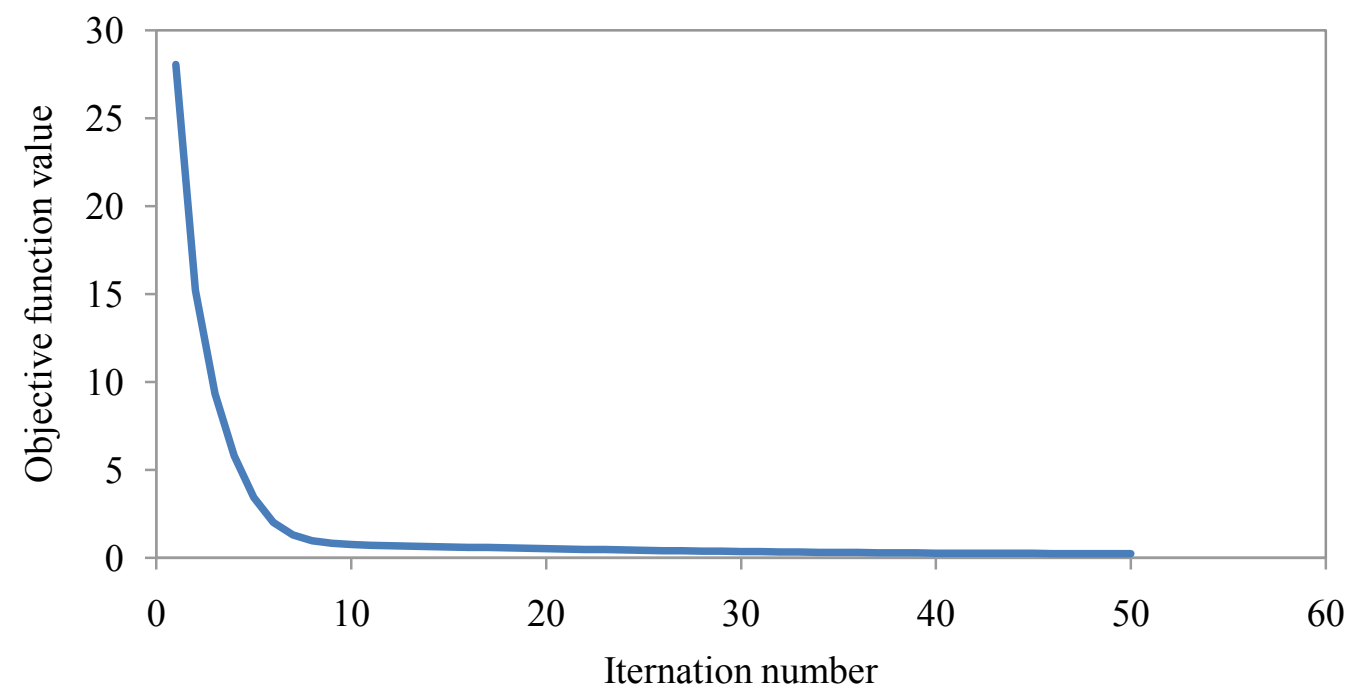

Figure 5.10 Performance of Zhou's dynamic O-D matrix estimation model.

Table 5.6 Comparison of traffic volume and speed based on DODE and Zhou's model.

\begin{tabular}{|c|r|r|r|r|}
\hline \multirow{2}{*}{$\begin{array}{l}\text { Screen } \\
\text { line }\end{array}$} & \multicolumn{2}{|c|}{ RMSPE for volume } & \multicolumn{3}{c|}{ RMSPE for speed } \\
\cline { 2 - 5 } & $\begin{array}{l}\text { After } \begin{array}{c}\text { Zhou's } \\
\text { model estimation }\end{array} \\
\text { After DODE }\end{array}$ & $\begin{array}{l}\text { After } \\
\text { model estimation }\end{array}$ & After DODE \\
\hline 1 & 0.016 & 0.009 & 0.198 & 0.181 \\
\hline 2 & 0.031 & 0.013 & 0.066 & 0.068 \\
\hline 3 & 0.013 & 0.008 & 0.182 & 0.181 \\
\hline 4 & 0.045 & 0.005 & 0.533 & 0.522 \\
\hline 5 & 0.068 & 0.018 & 0.414 & 0.406 \\
\hline 6 & 0.016 & 0.012 & 0.192 & 0.194 \\
\hline 7 & 0.100 & 0.027 & 0.401 & 0.403 \\
\hline 8 & 0.016 & 0.002 & 0.299 & 0.304 \\
\hline 9 & 0.021 & 0.001 & 0.336 & 0.331 \\
\hline 10 & 0.010 & 0.002 & 0.231 & 0.239 \\
\hline 11 & 0.002 & 0.007 & $\mathrm{X}$ & $\mathrm{X}$ \\
\hline 12 & 0.039 & 0.008 & $\mathrm{X}$ & $\mathrm{X}$ \\
\hline 13 & 0.005 & 0.005 & $\mathrm{X}$ & $\mathrm{X}$ \\
\hline 14 & 0.008 & 0.008 & $\mathrm{X}$ & $\mathrm{X}$ \\
\hline 15 & 0.010 & 0.005 & $\mathrm{X}$ & $\mathrm{X}$ \\
\hline 16 & 0.007 & 0.005 & $\mathrm{X}$ & $\mathrm{X}$ \\
\hline 17 & 0.006 & 0.004 & 0.285 & 0.283 \\
\hline 18 & 0.004 & 0 & & \\
\hline Avg. & 0.023 & 0.010 & & \\
\hline
\end{tabular}




\subsection{Traffic Flow Model Fine Tuning (TFMFT)}

The purpose of this section is to successively adjust the parameters in each traffic flow model to reduce speed deviation without increasing volume deviation. As mentioned in Section 3.5, the successive SPSA algorithm is used to calibrate each of the traffic models. Before using the algorithm, coefficients in the algorithm need to be predefined.

Spall (1998) recommended that, for the coefficients in gain sequences $a_{i}=a /(A+$ $i+1)^{\alpha}$ and $c_{i}=c /(i+1)^{\gamma}$ in the SPSA algorithm, the coefficients $\alpha$ and $\gamma$ should be around 0.602 and 0.101 . He also suggested that the basic \pm 1 Bernoulli distribution be used to describe the components of perturbation vectors. In this case study, through trial and error, the other parameters of the SPSA algorithm are set to $a=50, c=5$, and $\mathrm{A}=100$. The maximum number of iterations for the SPSA is set to 400 . For the calibration of each traffic flow model, the SPSA algorithm is switched to the next candidate traffic flow model if, (1) no better result is found in 30 consecutive iterations, or (2) the objective function value has reached the predefined level.

The performance of the successive SPSA algorithm for traffic flow model calibration is illustrated in Figure 5.11. Based on the figure, after about 300 iterations, a satisfying solution is found. After calibrating traffic flow models using the successive SPSA algorithm, there is improvement in the RMSPE for speed. The RMSPE for speed has been reduced from 0.28 to 0.17 , while the RMSPE for volume did not change significantly. The results are provided in Table 5.7, where " $\mathrm{X}$ " means no data are available because the detectors at the corresponding screenlines did not collect traffic speed data. 


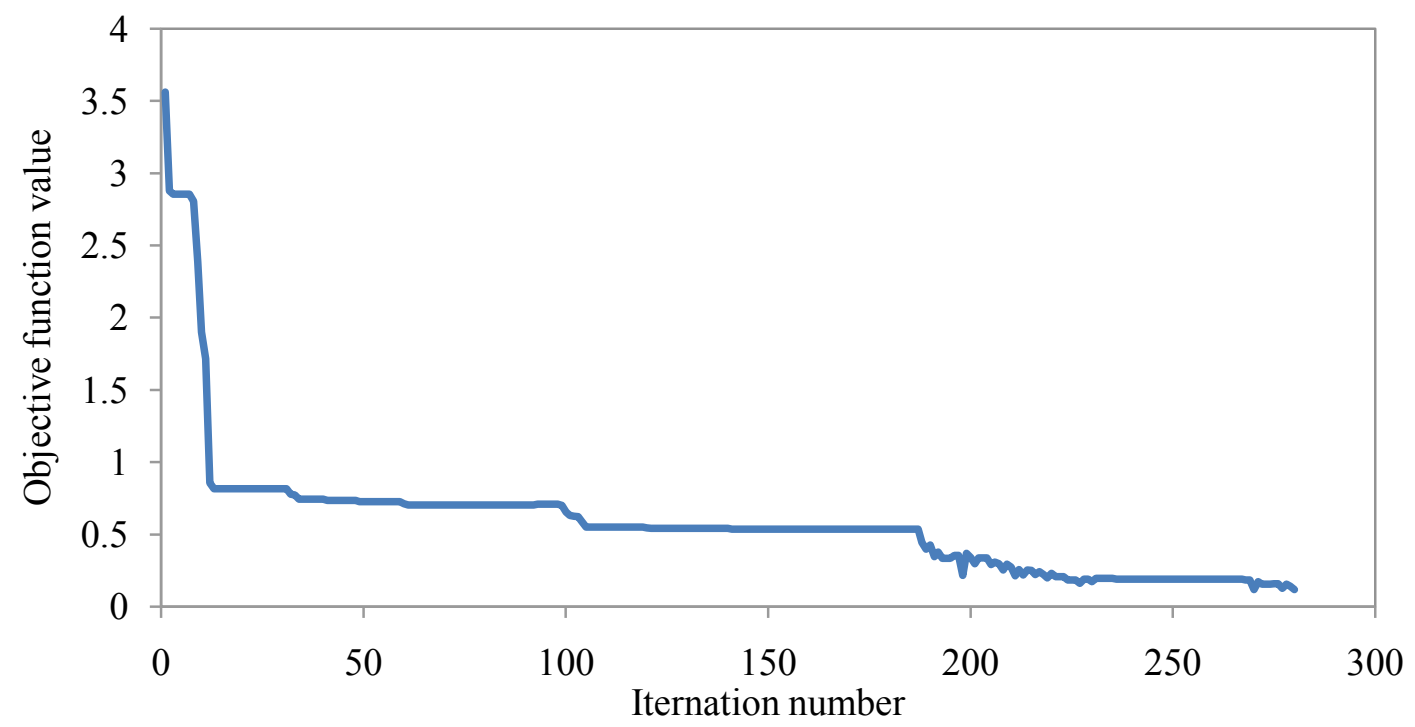

Figure 5.11 Performance of the successive SPSA algorithm for TFMFT.

Table 5.7 Traffic volume and speed comparison after TFMFT.

\begin{tabular}{|c|c|c|c|c|c|c|}
\hline \multirow[b]{2}{*}{$\begin{array}{l}\text { Screen } \\
\text { Line }\end{array}$} & \multicolumn{2}{|c|}{ Avg. Volume per 15 Minutes } & \multirow{2}{*}{$\begin{array}{l}\text { RMSPE } \\
\text { for } \\
\text { Volume }\end{array}$} & \multicolumn{2}{|c|}{ Avg. Speed per 15 Minutes } & \multirow{2}{*}{$\begin{array}{l}\text { RMSPE } \\
\text { for } \\
\text { Speed }\end{array}$} \\
\hline & Observed & After TFMFT & & Observed & After TFMFT & \\
\hline 1 & 1,148 & 1,164 & 0.02 & 45 & 49 & 0.16 \\
\hline 2 & 798 & 795 & 0.02 & 55 & 58 & 0.07 \\
\hline 3 & 1,376 & 1,375 & 0.01 & 45 & 50 & 0.16 \\
\hline 4 & 2,202 & 2,193 & 0.03 & 43 & 39 & 0.26 \\
\hline 5 & 2,133 & 2,039 & 0.05 & 48 & 47 & 0.24 \\
\hline 6 & 2,129 & 2,108 & 0.02 & 51 & 58 & 0.17 \\
\hline 7 & 1,869 & 1,972 & 0.06 & 42 & 46 & 0.12 \\
\hline 8 & 2,039 & 2,028 & 0.01 & 48 & 55 & 0.18 \\
\hline 9 & 2,066 & 2,049 & 0.01 & 44 & 51 & 0.18 \\
\hline 10 & 2,081 & 2,102 & 0.01 & 53 & 62 & 0.18 \\
\hline 11 & 174 & 174 & 0.00 & $\mathrm{X}$ & $\mathrm{X}$ & $\mathrm{X}$ \\
\hline 12 & 59 & 59 & 0.01 & $\mathrm{X}$ & $\mathrm{X}$ & $\mathrm{X}$ \\
\hline 13 & 180 & 180 & 0.00 & $\mathrm{X}$ & $\mathrm{X}$ & $\mathrm{X}$ \\
\hline 14 & 98 & 98 & 0.00 & $\mathrm{X}$ & $\mathrm{X}$ & $\mathrm{X}$ \\
\hline 15 & 138 & 137 & 0.00 & $\mathrm{X}$ & $\mathrm{X}$ & $X$ \\
\hline 16 & 192 & 193 & 0.01 & $\mathrm{X}$ & $X$ & $X$ \\
\hline 17 & 188 & 188 & 0.00 & $X$ & $\mathrm{X}$ & $\mathrm{X}$ \\
\hline 18 & 181 & 181 & 0.00 & $\mathrm{X}$ & $\mathrm{X}$ & $\mathrm{X}$ \\
\hline Avg. & 1,058 & 1,058 & 0.01 & 47.4 & 51.5 & 0.17 \\
\hline
\end{tabular}




\subsection{Iterations between DODE and TFMFT}

Based on the proposed methodology, DODE and TFMFT are iterated to obtain the best solution. For this case study, only two iterations are needed to reach a stable solution. The final calibrated traffic flow models are presented in Table 5.8. Table 5.9 presents the comparison between the observed volume and speed and the assignment volume and speed, where ODTFM represents the combination between the dynamic O-D matrix estimation and traffic flow model fine-tuning. It can be seen that the average RMSPE for volume is 0.01 . The average RMSPE for speed is 0.130 .

Table 5.8 Results of traffic flow model calibration.

\begin{tabular}{|c|c|c|c|c|c|c|}
\hline model \# & $\alpha$ & $U_{f}$ & $V_{0}$ & $V_{f}$ & $K_{j}$ & $K_{b}$ \\
\hline 1 & 1.38 & 47.78 & 8.80 & 50.70 & 200 & 10.19 \\
\hline 2 & 2.49 & 55.71 & 10.35 & 71.34 & 200 & 22.42 \\
\hline 3 & 1.46 & 50.17 & 10.99 & 56.08 & 200 & 18.35 \\
\hline 4 & 1.22 & 56.38 & 9.12 & 61.53 & 200 & 16.27 \\
\hline 5 & 2.85 & 53.66 & 12.51 & 64.73 & 200 & 16.04 \\
\hline 6 & 2.23 & 56.29 & 11.43 & 64.81 & 200 & 15.00 \\
\hline 7 & 3.46 & 51.11 & 9.59 & 63.97 & 200 & 15.00 \\
\hline 8 & 1.64 & 53.36 & 10.01 & 60.04 & 200 & 16.74 \\
\hline 9 & 1.82 & 53.34 & 9.82 & 59.97 & 200 & 15.00 \\
\hline 10 & 1.34 & 50.05 & 9.51 & 56.28 & 200 & 20.24 \\
\hline
\end{tabular}

The reason a higher RMSPE for speed is obtained, compared to that for volume, may be attributed to three reasons: (1) erroneous assignment of the traffic flow model to the closed links, since more traffic data need to be collected to calibrate the traffic flow model in other links; (2) low-performance of the stochastic algorithm; and/or (3) the mesoscopic simulation model embedded in the DTA simulator cannot simulate changes of speed due to some traffic conditions, such as traffic weaving before and after an interchange. Further study is needed to address these problems. 
Table 5.9 Final assignment traffic volume and traffic speed comparison.

\begin{tabular}{|c|c|c|c|c|c|c|}
\hline \multirow{2}{*}{$\begin{array}{l}\text { Screen } \\
\text { Line }\end{array}$} & \multicolumn{2}{|c|}{ Avg. Volume per 15 Minutes } & \multirow{2}{*}{$\begin{array}{l}\text { RMSPE } \\
\text { Volume }\end{array}$} & \multicolumn{2}{|c|}{ Avg. Speed per 15 Minutes } & \multirow{2}{*}{$\begin{array}{l}\text { RMSPE } \\
\text { Speed }\end{array}$} \\
\hline & Observed & After ODTFM & & Observed & After ODTFM & \\
\hline 1 & 1,148 & 1,158 & 0.02 & 45 & 46 & 0.12 \\
\hline 2 & 798 & 796 & 0.01 & 55 & 58 & 0.07 \\
\hline 3 & 1,376 & 1,375 & 0.01 & 45 & 46 & 0.11 \\
\hline 4 & 2,202 & 2,190 & 0.03 & 43 & 40 & 0.26 \\
\hline 5 & 2,133 & 2,040 & 0.05 & 48 & 45 & 0.21 \\
\hline 6 & 2,129 & 2,098 & 0.02 & 51 & 52 & 0.09 \\
\hline 7 & 1,869 & 1,963 & 0.05 & 42 & 46 & 0.11 \\
\hline 8 & 2,039 & 2,034 & 0.01 & 48 & 50 & 0.07 \\
\hline 9 & 2,066 & 2,051 & 0.01 & 44 & 46 & 0.11 \\
\hline 10 & 2,081 & 2,101 & 0.01 & 53 & 57 & 0.11 \\
\hline 11 & 174 & 174 & 0.00 & $X$ & $X$ & $\mathrm{X}$ \\
\hline 12 & 59 & 59 & 0.01 & $\mathrm{X}$ & $X$ & $X$ \\
\hline 13 & 180 & 180 & 0.00 & X & X & X \\
\hline 14 & 98 & 98 & 0.00 & $X$ & $X$ & $\mathrm{X}$ \\
\hline 15 & 138 & 137 & 0.00 & $\mathrm{X}$ & $X$ & $\mathrm{X}$ \\
\hline 16 & 192 & 193 & 0.01 & $\mathrm{X}$ & $\mathrm{X}$ & $\mathrm{X}$ \\
\hline 17 & 188 & 188 & 0.00 & $\mathrm{X}$ & $\mathrm{X}$ & $\mathrm{X}$ \\
\hline 18 & 181 & 181 & 0.00 & $X$ & $\mathrm{X}$ & $\mathrm{X}$ \\
\hline Avg. & 1,058 & 1,056 & 0.01 & 47.4 & 48.6 & 0.13 \\
\hline
\end{tabular}

Figure 5.12 plots the observed volume against the assignment volume based on the calibrated traffic flow model and estimated dynamic O-D matrix. In the figure, the fit line is a red color, indicating the closeness between observed volume and assignment volume. Based on the equation of the fit line, it can be seen that the link volume generated by the estimated O-D matrix is close to the observed volume. Figure 5.13 illustrates the comparison between the observed speed and the final assignment speed based on the estimated O-D matrix. According to the figure, it can be seen that the fitness between them is not satisfying, which is indicated in Table 5.9. 


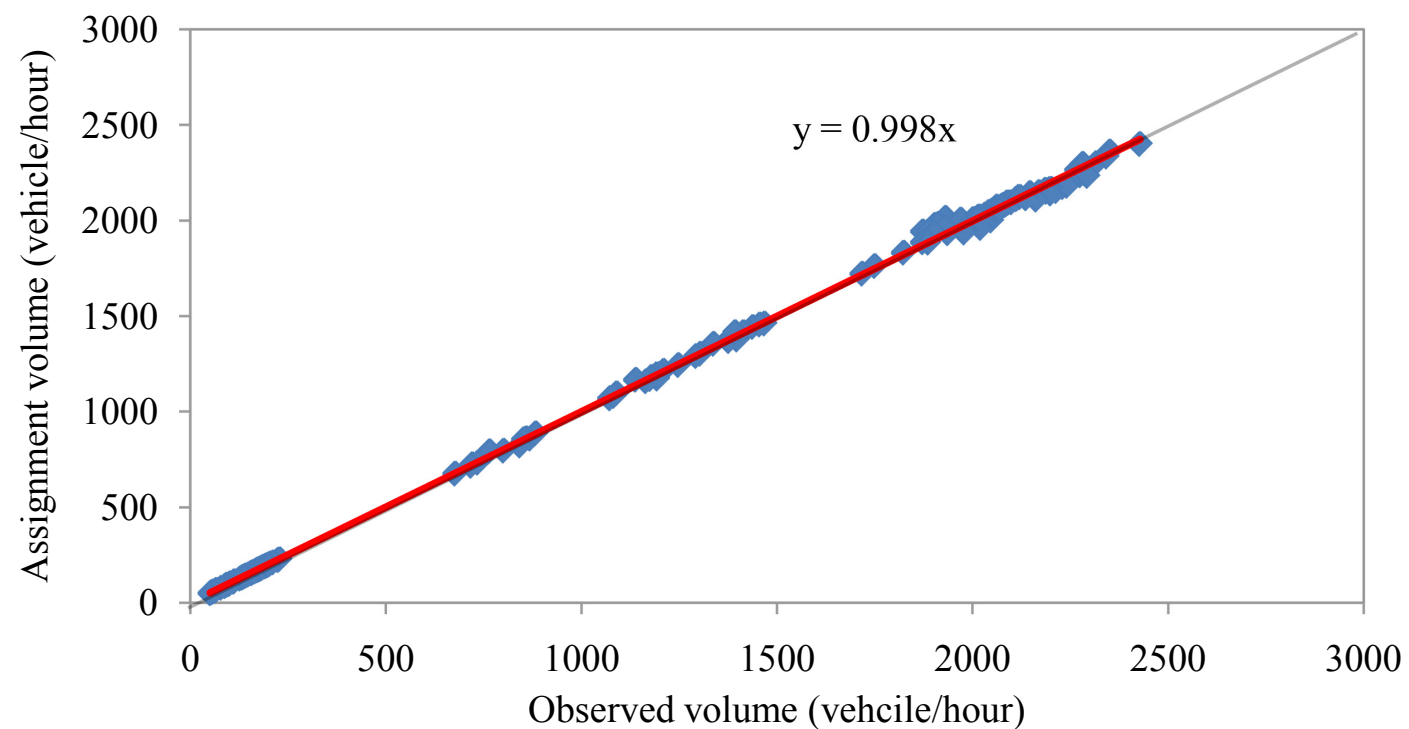

Figure 5.12 Observed volume versus final assignment volume.

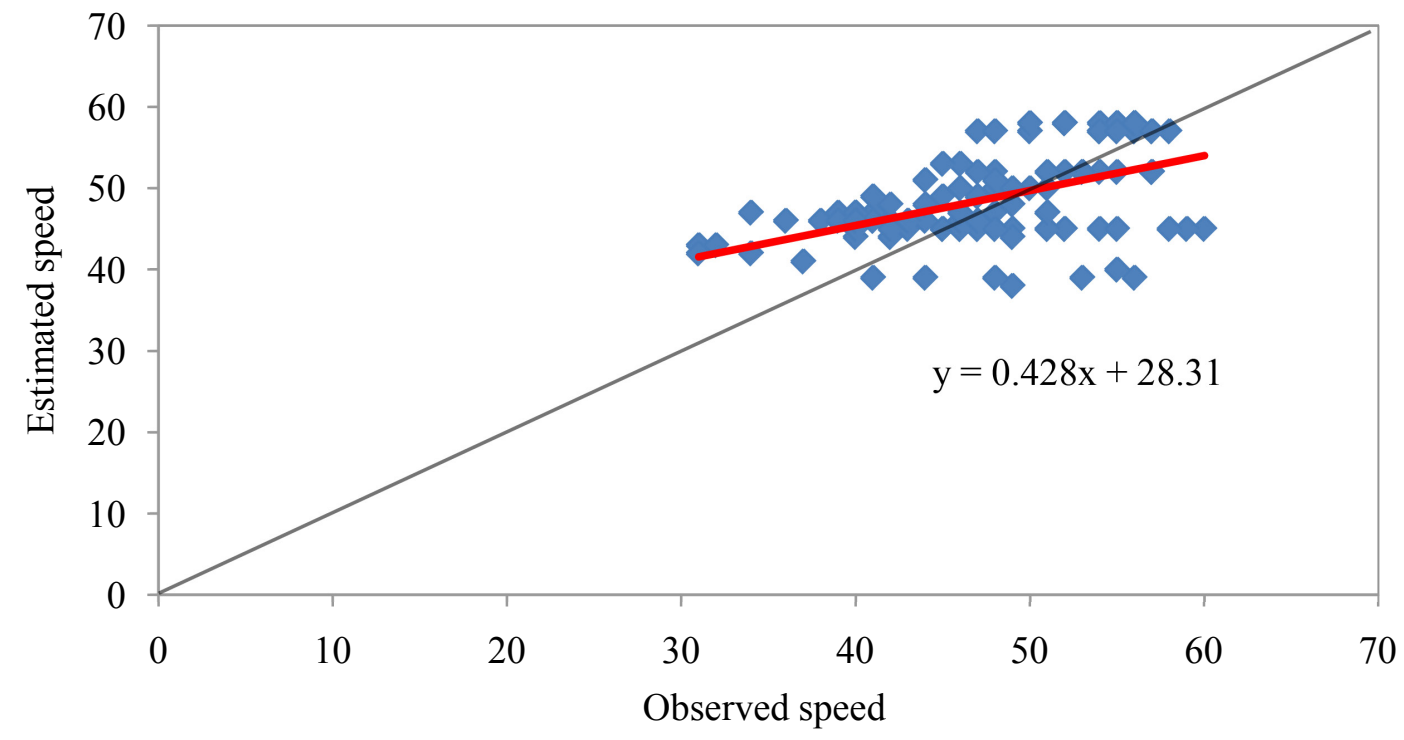

Figure 5.13 Observed speed versus final assignment speed.

\section{$5.8 \quad$ Summary}

This chapter presents a case study for a dynamic O-D matrix estimation on a segment of I-95. The traffic flow model calibration (TFMC) model employs linear regression to calibrate the parameters of traffic flow models in the DTA model. The calibration is reasonable and the average $\mathrm{R}$ square is around 0.70 . The initial O-D estimation (IODE) 
model can produce a good initial input and reduce the computational time for the dynamic O-D estimation (DODE) model. It can also reduce the objective function value from 28.05 to 1.33 in about four iterations.

With the estimated initial O-D matrix, the dynamic O-D estimation (DODE) model can obtain the improved result in four iterations, and the objective function value drops from 1.33 to 0.025 . By comparison, it takes 50 iterations for Zhou's dynamic O-D matrix estimation model to reach the final objective function value of 0.250 . Therefore, the proposed models demonstrate a better performance than Zhou's model in this case study.

The traffic flow model fine-tuning model (TFMFT) uses the successive SPSA algorithm to fine-tune the traffic flow model of the DTA simulator. Instead of calibrating the traffic flow models simultaneously, this study uses a successive strategy to calibrate them one-by-one. This strategy can reduce the dimensions of the unknown variables significantly, thus improving the performance of the original SPSA algorithm. The RMSPE for speed drops from 0.280 to 0.120 . When the estimated dynamic O-D matrix is loaded onto the calibrated road network, the average RMSPE for volume is 0.010 , and the RMSPE for speed is 0.130 . 


\section{CHAPTER 6}

\section{A CASE STUDY OF JACKSONVILLE}

This case study is designed to demonstrate the performance of the proposed methodology framework for a large-scale network. The case study is performed on the road network for Jacksonville, Florida. The road network and initial O-D demand are extracted from the integrated Florida statewide model (FLSWM). The basic time interval for the dynamic O-D matrix is set at 15 minutes. The number of basic time intervals is eight for the dynamic O-D matrix, with the study conducted from 18:00 to 20:00 on a typical work day. In this chapter, the road network and initial O-D matrix are prepared in Section 6.1, traffic flow models are calibrated in Section 6.2, the initial O-D matrix is estimated in Section 6.3, and the dynamic O-D matrix is estimated in Section 6.4.

\subsection{Subarea Road Network and Initial Traffic Demand Preparation}

CUBE is used to extract the subarea network and initial period O-D matrix from FLSWM. The extracted subarea network is presented in Figure 6.1. The subarea network includes interstate highway I-10, I-95, and I-295 and some arterials. There are a total of 1,238 links and 143 zones in the subarea network. Based on the traffic volume distribution factors, the extracted period O-D matrix is then factorized into a series of sub-period O-D matrices for the input of initial O-D estimation in Section 6.3. 


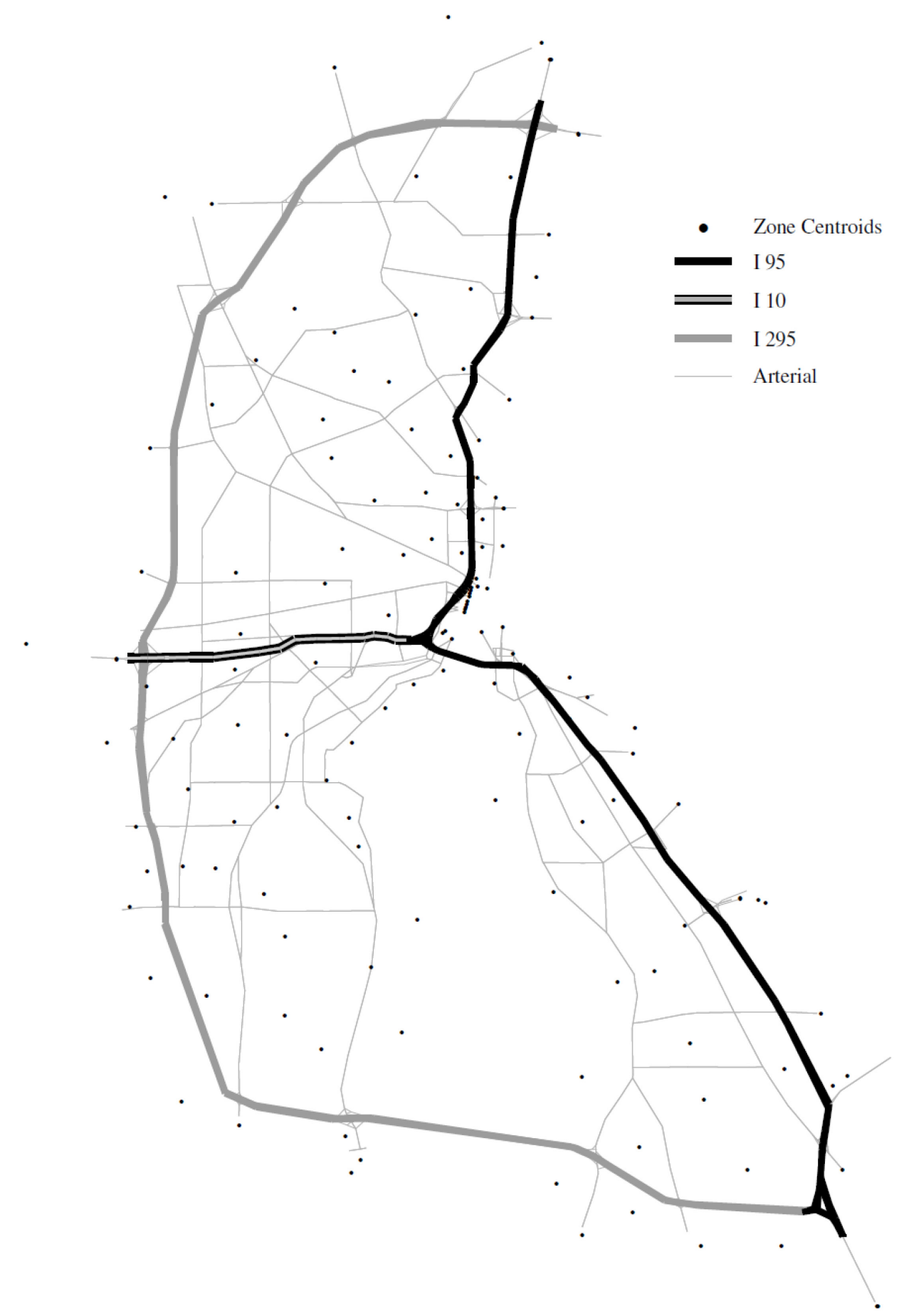

Figure 6.1 Subarea road network in Jacksonville, FL. 


\subsection{Traffic Data Preparation and Traffic Flow Model Calibration}

There are a total of 35 detectors selected to provide traffic measurement data for the dynamic O-D matrix estimation. The detectors are listed in Table 6.1, with the locations of these detectors specified in Figure 6.2. Traffic data including speed, volume, and occupancy are collected from these detectors within a time interval of 15 minutes.

Table 6.1 Detector and road link.

\begin{tabular}{|c|c|c|c|c|c|c|c|}
\hline & & \multicolumn{2}{|c|}{ Link Node } & & & \multicolumn{2}{c|}{ Link Node } \\
\cline { 7 - 8 } Num. & Detector & A & B & Num. & Detector & A & B \\
\hline 1 & 220022 & 118541 & 118542 & 19 & 200052 & 118741 & 119989 \\
\hline 2 & 220122 & 118488 & 119962 & 20 & 200042 & 122802 & 119994 \\
\hline 3 & 220142 & 120536 & 118544 & 21 & 210312 & 120057 & 122036 \\
\hline 4 & 220202 & 118483 & 118478 & 22 & 210362 & 119140 & 120060 \\
\hline 5 & 220362 & 118547 & 118475 & 23 & 210442 & 121604 & 121925 \\
\hline 6 & 220432 & 118549 & 118550 & 24 & 210632 & 119151 & 119152 \\
\hline 7 & 220562 & 118465 & 119950 & 25 & 210692 & 119154 & 119155 \\
\hline 8 & 220602 & 118551 & 118552 & 26 & 210711 & 121013 & 120788 \\
\hline 9 & 220631 & 122230 & 120971 & 27 & 210511 & 119133 & 119136 \\
\hline 10 & 220551 & 121413 & 120520 & 28 & 210371 & 119119 & 119120 \\
\hline 11 & 220491 & 118435 & 118436 & 29 & 210211 & 119122 & 119123 \\
\hline 12 & 220382 & 120512 & 120513 & 30 & 210171 & 119147 & 121602 \\
\hline 13 & 220311 & 118440 & 120514 & 31 & 210041 & 121457 & 118668 \\
\hline 14 & 220131 & 118442 & 118443 & 32 & 200091 & 118674 & 118676 \\
\hline 15 & 220071 & 118491 & 118494 & 33 & 200141 & 118706 & 118677 \\
\hline 16 & 220011 & 120970 & 118445 & 34 & 200201 & 118680 & 118681 \\
\hline 17 & 200132 & 118750 & 118705 & 35 & 200242 & 121466 & 118718 \\
\hline 18 & 200082 & 118751 & 118752 & & & & \\
\hline
\end{tabular}




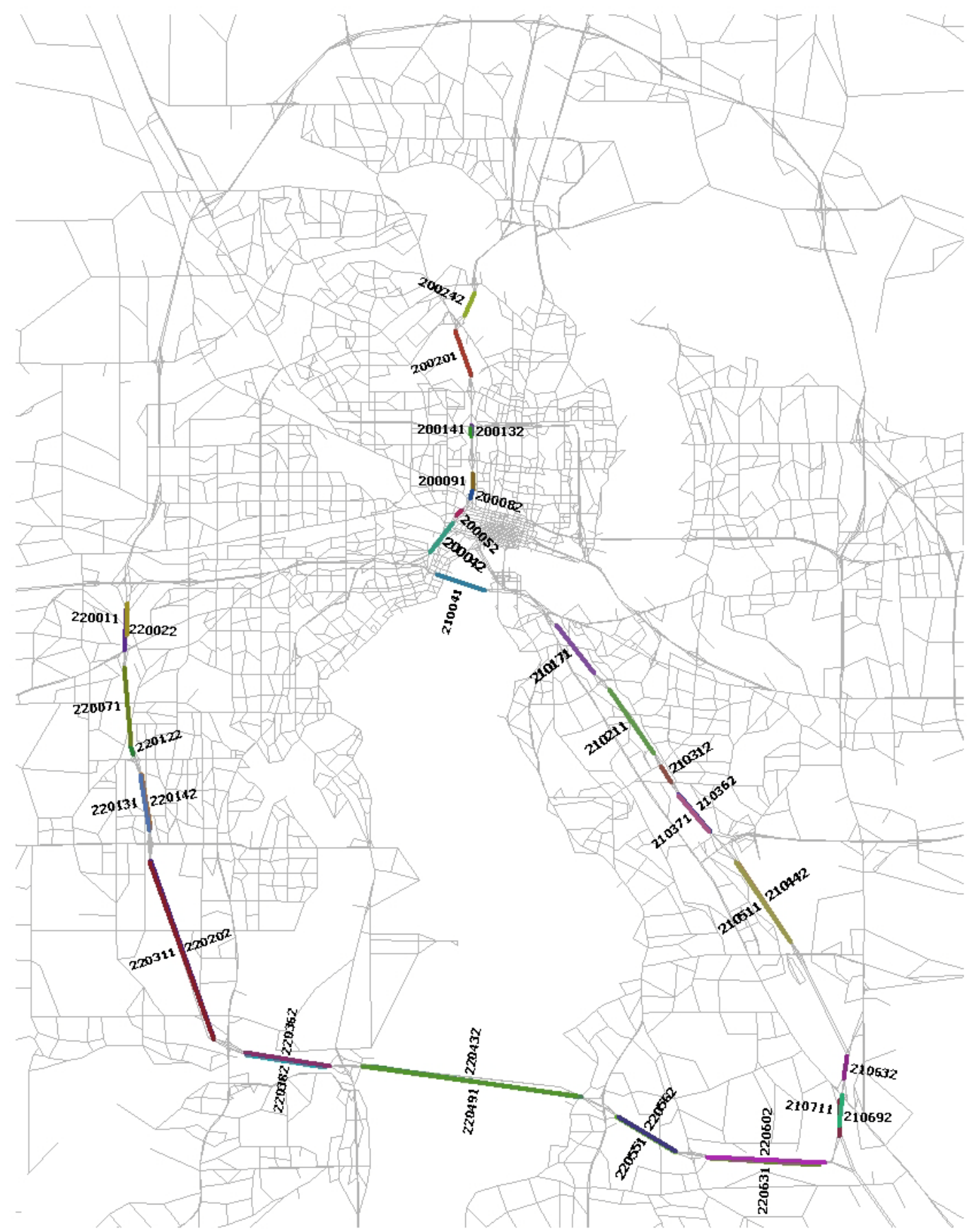

Figure 6.2 The location of detectors in the subarea road network.

According to the availability of traffic data, a total of ten traffic flow models are calibrated based on the proposed linear regression algorithm, as presented in Table 6.2. 
Table 6.2 Traffic flow model parameters.

\begin{tabular}{|c|c|c|c|c|c|c|c|}
\hline Model & $U_{f}$ & $K_{b}$ & $V_{f}$ & $V_{0}$ & $K_{j}$ & $\alpha$ & $\mathrm{R}^{2}$ \\
\hline 1 & 52 & 28 & 112 & 6 & 200 & 5.59 & 0.70 \\
\hline 2 & 65 & 22 & 106 & 6 & 200 & 4.59 & 0.88 \\
\hline 3 & 62 & 33 & 133 & 6 & 200 & 4.48 & 0.85 \\
\hline 4 & 65 & 18 & 99 & 6 & 200 & 4.12 & 0.83 \\
\hline 5 & 69 & 20 & 126 & 6 & 200 & 6.11 & 0.64 \\
\hline 6 & 69 & 27 & 130 & 6 & 200 & 4.71 & 0.75 \\
\hline 7 & 64 & 23 & 110 & 6 & 200 & 4.75 & 0.83 \\
\hline 8 & 70 & 25 & 103 & 6 & 200 & 3.05 & 0.79 \\
\hline 9 & 68 & 27 & 170 & 6 & 200 & 6.74 & 0.91 \\
\hline 10 & 60 & 23 & 90 & 6 & 200 & 3.60 & 0.85 \\
\hline
\end{tabular}

\subsection{Initial O-D Matrix Estimation (IODE)}

The initial O-D matrix estimation includes two parts: the static initial O-D matrix estimation and the dynamic O-D estimation. The static initial O-D matrix estimation (SIODE) is performed with the aid of the CUBE ANALYST program. CUBE ANALYST requires road network, traffic count, initial O-D matrix, and so forth as inputs. The initial estimation methodology flow chart is presented in Figure 5.3.

The extracted subarea O-D matrix is a daily O-D matrix, which needs to be factorized into a sequence of 15 minutes based on O-D matrices to be used as input for CUBE ANALYST. The daily O-D matrix is factorized based on the ratio of observed traffic volume in a certain time interval to the total observed traffic volume.

The initial O-D matrices are estimated based on the SIODE model. Like the previous case study in Chapter 5, RMSPE for volume is used to evaluate SIODE result. In Figure 6.3, the solid line represents the RMSPE between observed volume and static assignment volume based on the initial demand for the 35 detectors, and the dashed line stands for the RMSPE between observed volume and static assignment volume based on the estimated demand for the 35 detectors. The average RMSPE is reduced from 0.369 to 
0.111 as the result of SIODE. The figure demonstrates that the SIODE estimation improves the accuracy of the initial O-D matrix.

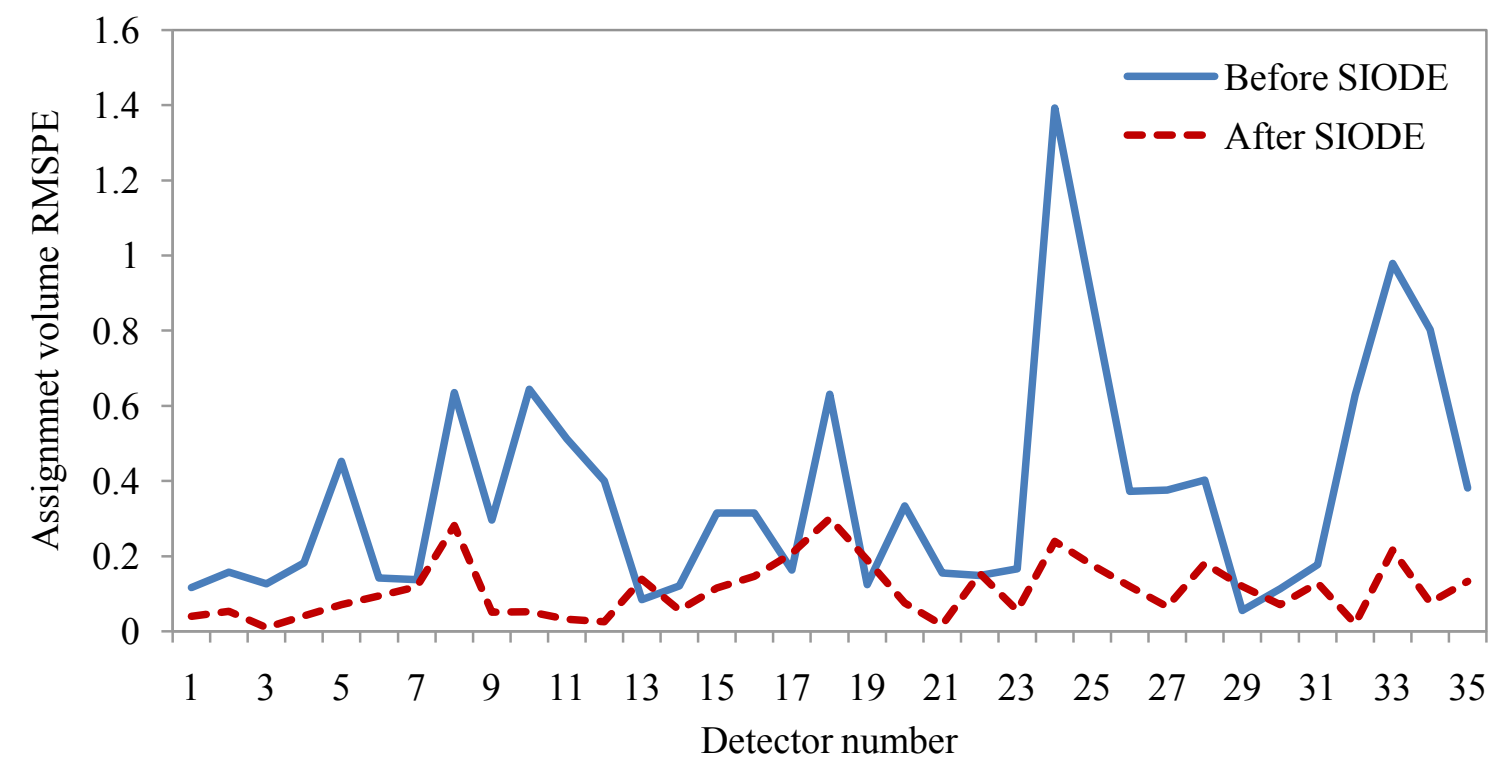

Figure 6.3 Comparison between assignment volume before-and-after SIODE.

SIODE depends on static assignment methods. The estimated O-D matrix needs to be further tuned based on dynamic traffic assignment methods. The DIODE model is used to further tune the estimated O-D matrix. The two proposed sub models in DIODE are used, and their performances are illustrated in Figures 6.4 and 6.5. Sub-model 1 reaches convergence after three iterations, and the objective function value drops from 400 to 48 . Sub model 2 reaches convergence after six iterations, and the objective function value drops from 48 to 45 . The DIODE model also proves advantageous in saving computation time, since it contains only a few variables. In this case study, Submodel 1 has one variable, and Sub-model 2 has eight variables. 


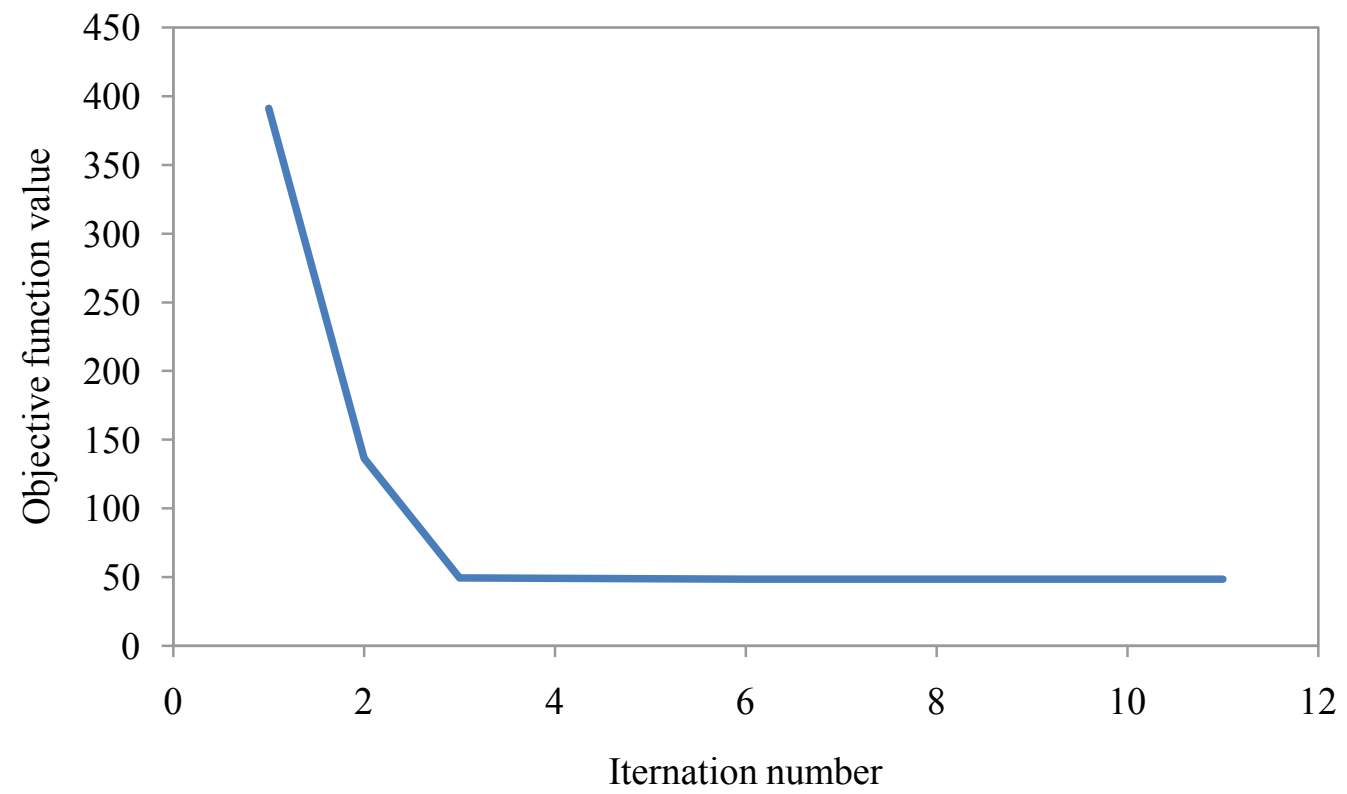

Figure 6.4 Performance of DIODE Sub-model 1.

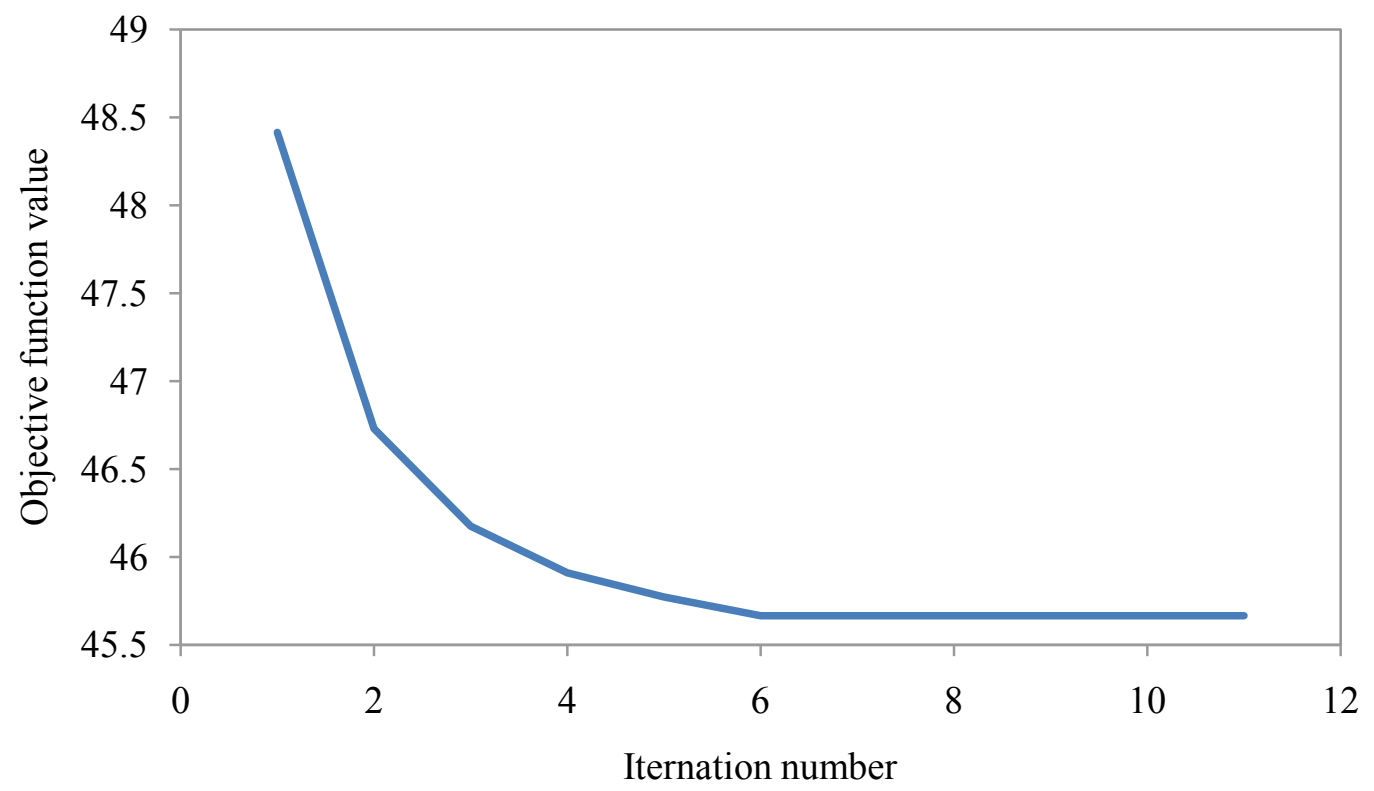

Figure 6.5 Performance of DIODE Sub-model 2.

With the aid of the DIODE model, the estimation result is improved, as is illustrated in Figure 6.6. In the figure, the solid line represents the RMSPE for volume before DIODE. The dashed line stands for the RMSPE of volume after DIODE. The average RMSPE for volume is reduced from 1.044 to 0.362 . It should be mentioned that 
the RMSPE for volume before DIODE in Figure 6.6 is different from that in Figure 6.3, because the assignment traffic volume is obtained from the static assignment in Figure 6.3 and from the dynamic assignment in Figure 6.6.

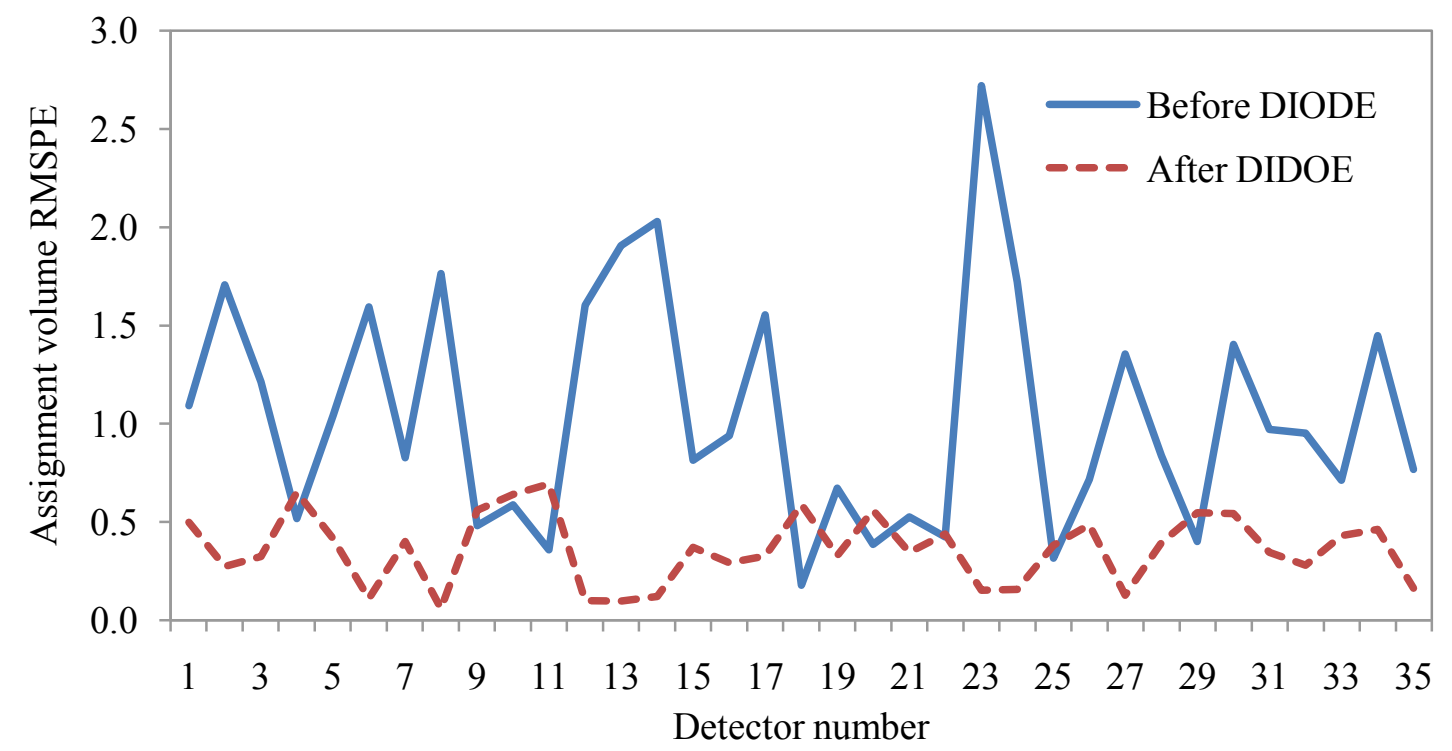

Figure 6.6 Comparison between assignment volume before-and-after DIODE.

\subsection{Dynamic O-D Matrix Estimation (DODE)}

After the initial O-D matrix estimation, the proposed dynamic O-D matrix estimation (DODE) model is used to estimate the dynamic O-D demand. Since there are more unknown variables (dynamic O-D pairs, $147 \times 147 \times 12$ ) than those of available constraint traffic information (available traffic measurement $35 \times 8 \times 2$ ), extra constraints are needed for the DODE model.

In this case study, additional capacity constraints are used. The assumption is that a traffic analysis zone (TAZ) will not produce or attract more trips than those of the road network connected with the TAZ can afford within the whole study periods. This idea can be illustrated in Figure 6.7, where $\mathrm{A}$ and $\mathrm{H}$ represent two different TAZs, AB and $\mathrm{GH}$ are the links directly connected with the TAZs, and $\mathrm{BC}, \mathrm{BD}, \mathrm{EC}$, and $\mathrm{EG}$ are 
connected with link $\mathrm{AB}$ and link GH. In the DTA simulator DYNASMART, usually a very large capacity is assigned to link $\mathrm{AB}$ and $\mathrm{GH}$, also called the generation link, so that the capacity bottlenecks often occur in link BD and BC for TAZ A, and in link EG and FG for TAZ H. Thus, the production constraint for TAZ A is the traffic production of $\mathrm{TAZ} \mathrm{A} \leq$ capacity of link BC plus capacity of link BD. In the same way, the attraction constraint for TAZ $\mathrm{H}$ is attraction of TAZ $\mathrm{H} \leq$ capacity of link EG plus capacity of link FG.

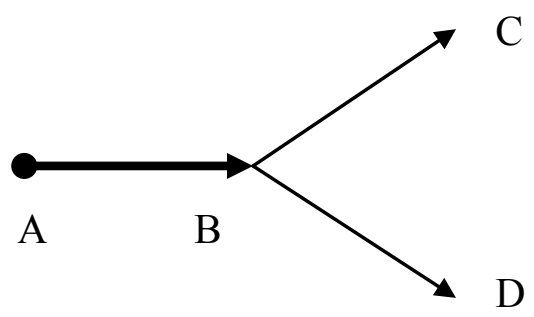

(a)

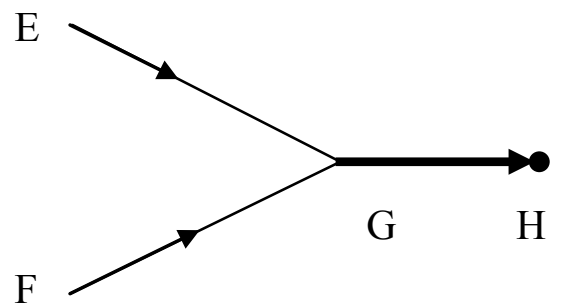

(b)

Figure 6.7 Capacity constrain.

Before using this capacity constraint, users need to make sure that there is no significant difference between the simulation and real road network in the connectors between TAZs and major roads; otherwise, the constraint may not reflect the real traffic conditions. In this study, with the proposed capacity constraints, the DODE model reaches a convergent solution point after 10 iterations, as illustrated in Figure 6.8, and the objective function value drops from 45.213 to 0.714 . Figure 6.9 shows a comparison of RMSPE for volume before-and-after DODE. Based on the figure, the RMSPE of volume is reduced significantly. The average RMSPE for volume drops from 0.362 to 0.045 through the DODE. 


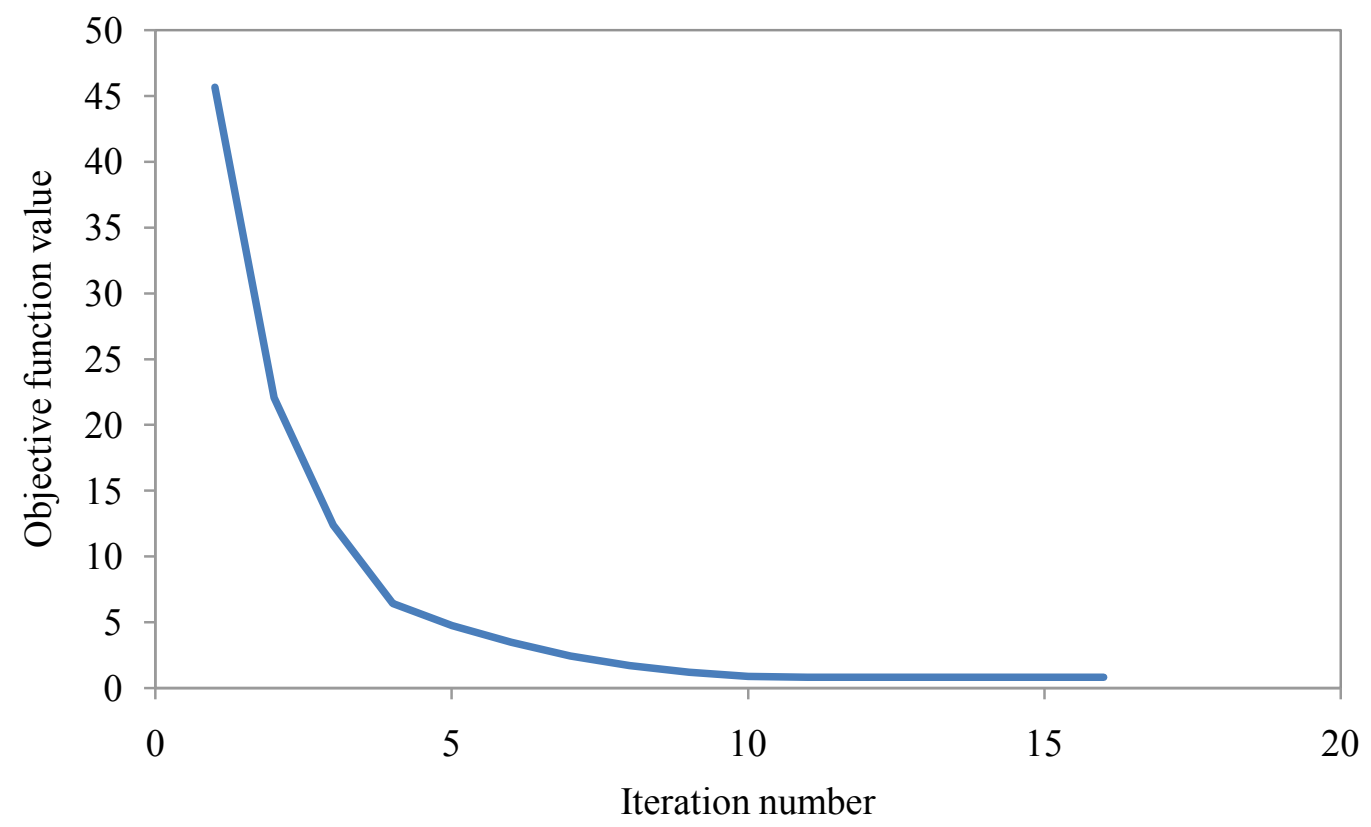

Figure 6.8 Performance of dynamic O-D matrix estimation (DODE).

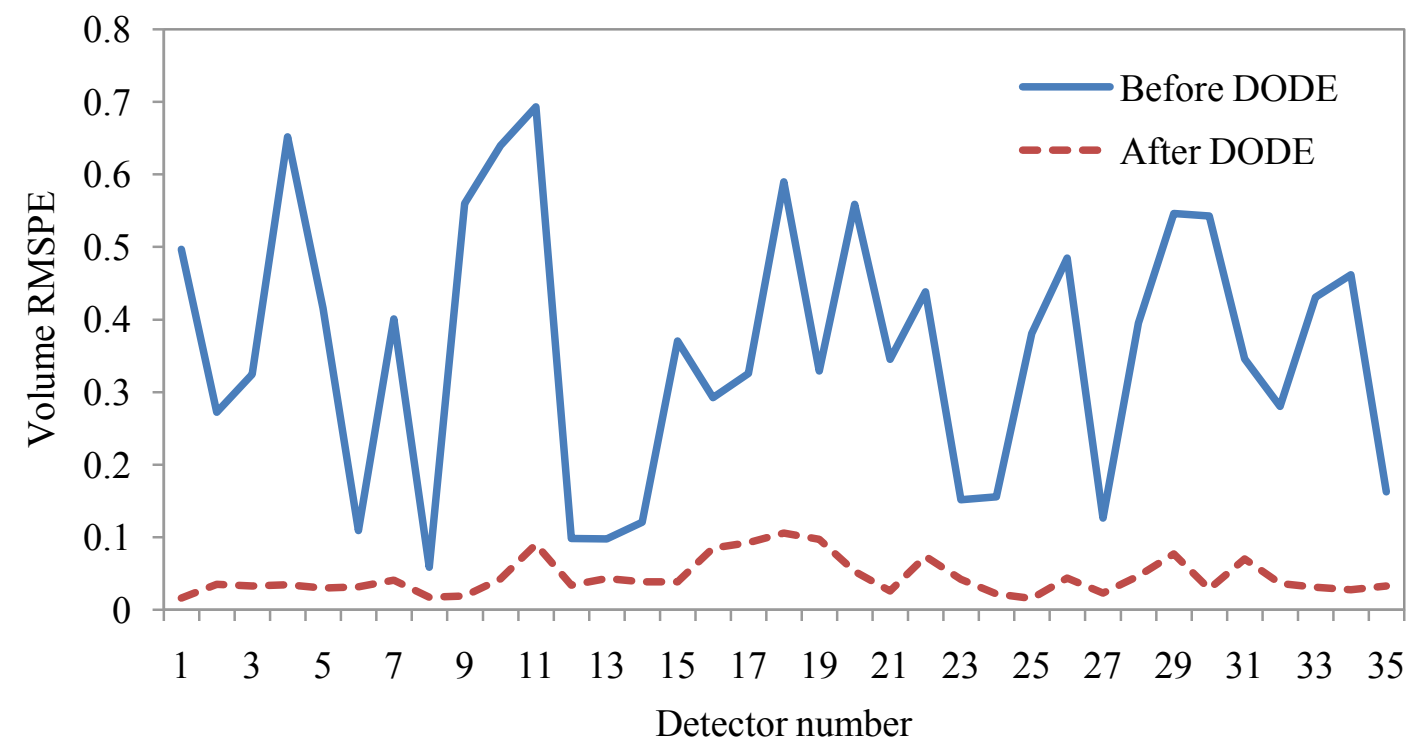

Figure 6.9 Comparison between assignment volume before-and-after DODE.

Figure 6.10 compares the observed volumes and assignment volumes before DODE and Figure 6.11, after DODE. By comparing Figure 6.10 with Figure 6.11, one can conclude that the estimation result is reasonable, and that the real traffic count can be reflected by assigning the estimated dynamic O-D matrix to the network. 


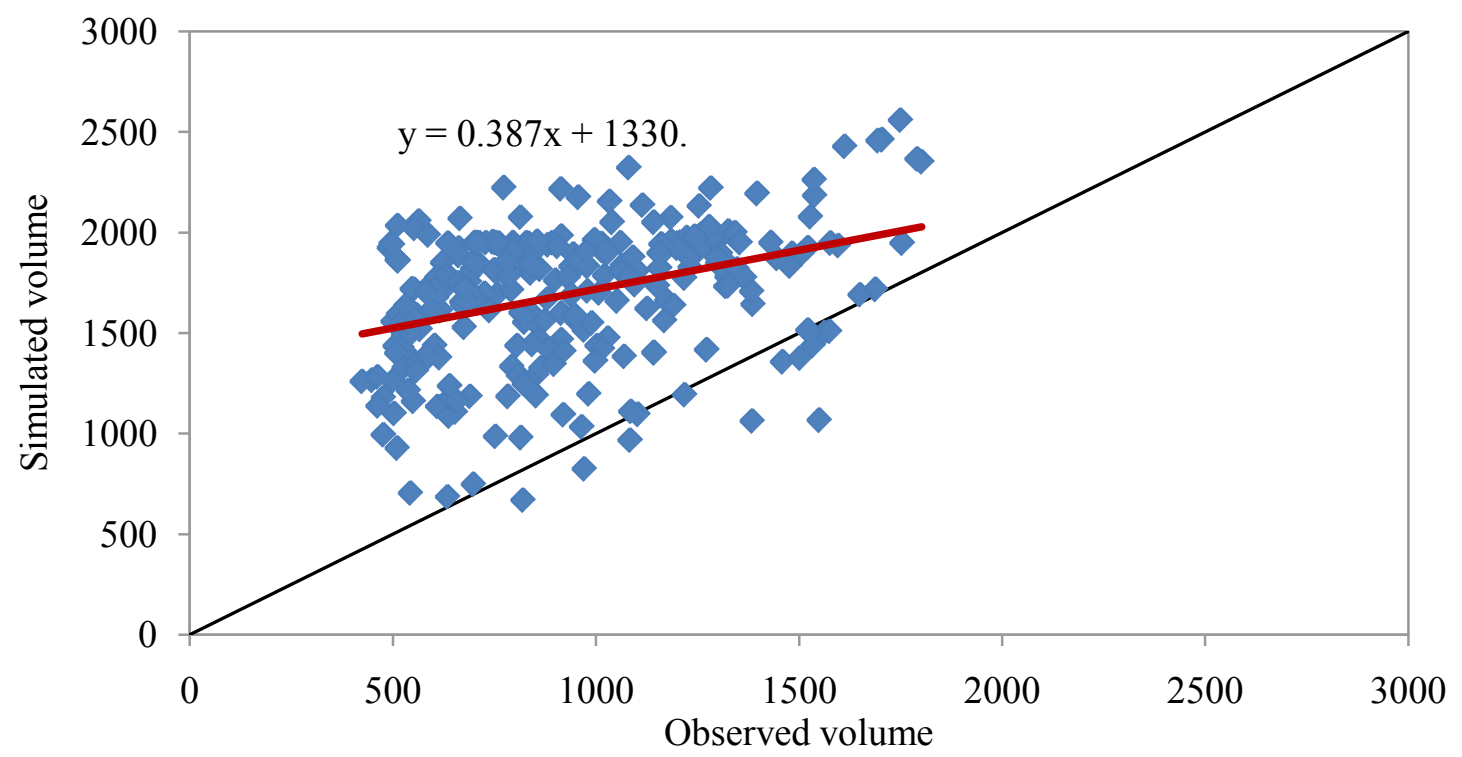

Figure 6.10 Assignment volumes versus observed volumes before SIODE.

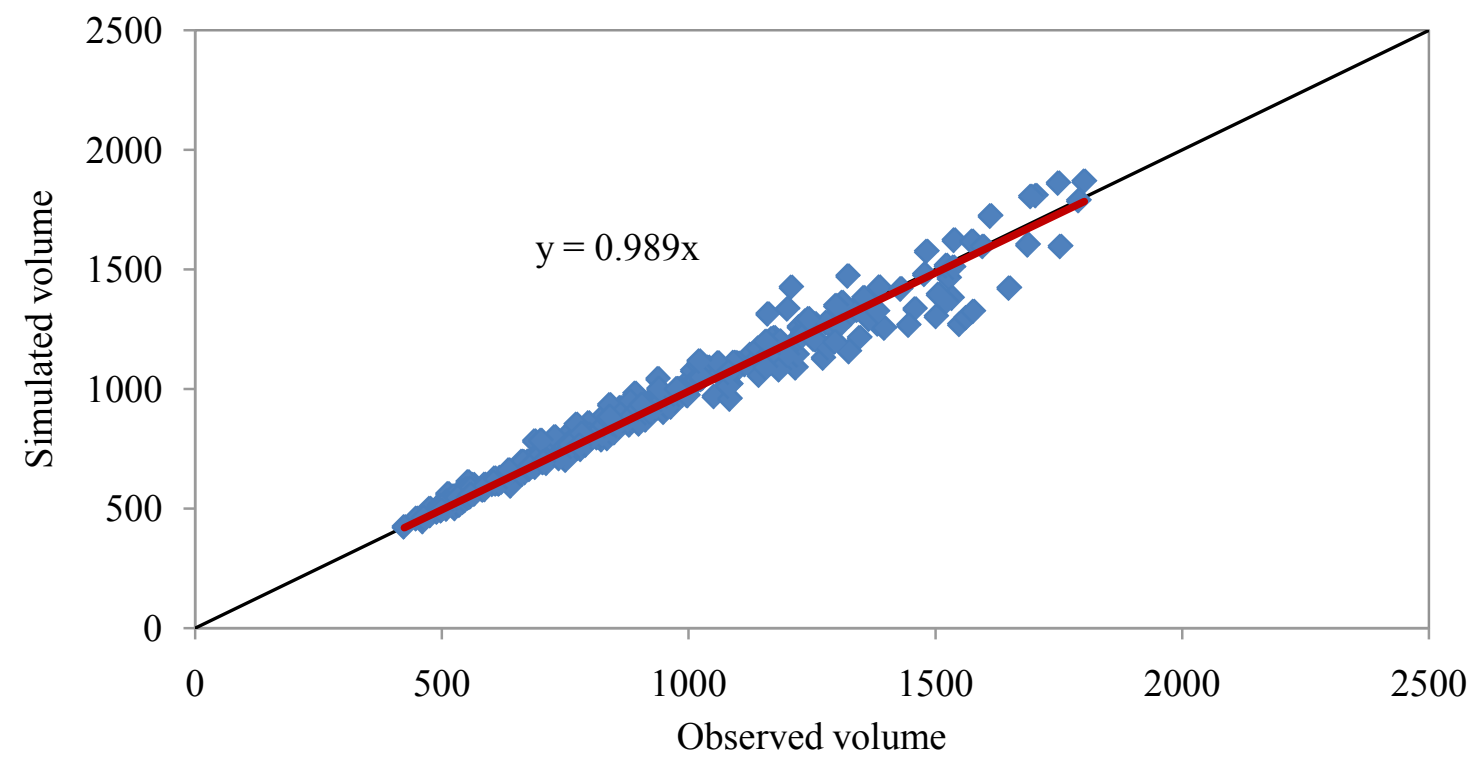

Figure 6.11 Assignment volumes versus observed volumes after DODE.

Figure 6.12 shows a comparison of RMSPE for speed before-and-after applying the proposed models. Although there is much improvement on the RMSPE for speed, which has been reduced from 0.410 to 0.110 , more work is needed since the RMSPE for the speed of some detectors is still large (around 0.5 after using the proposed models). 


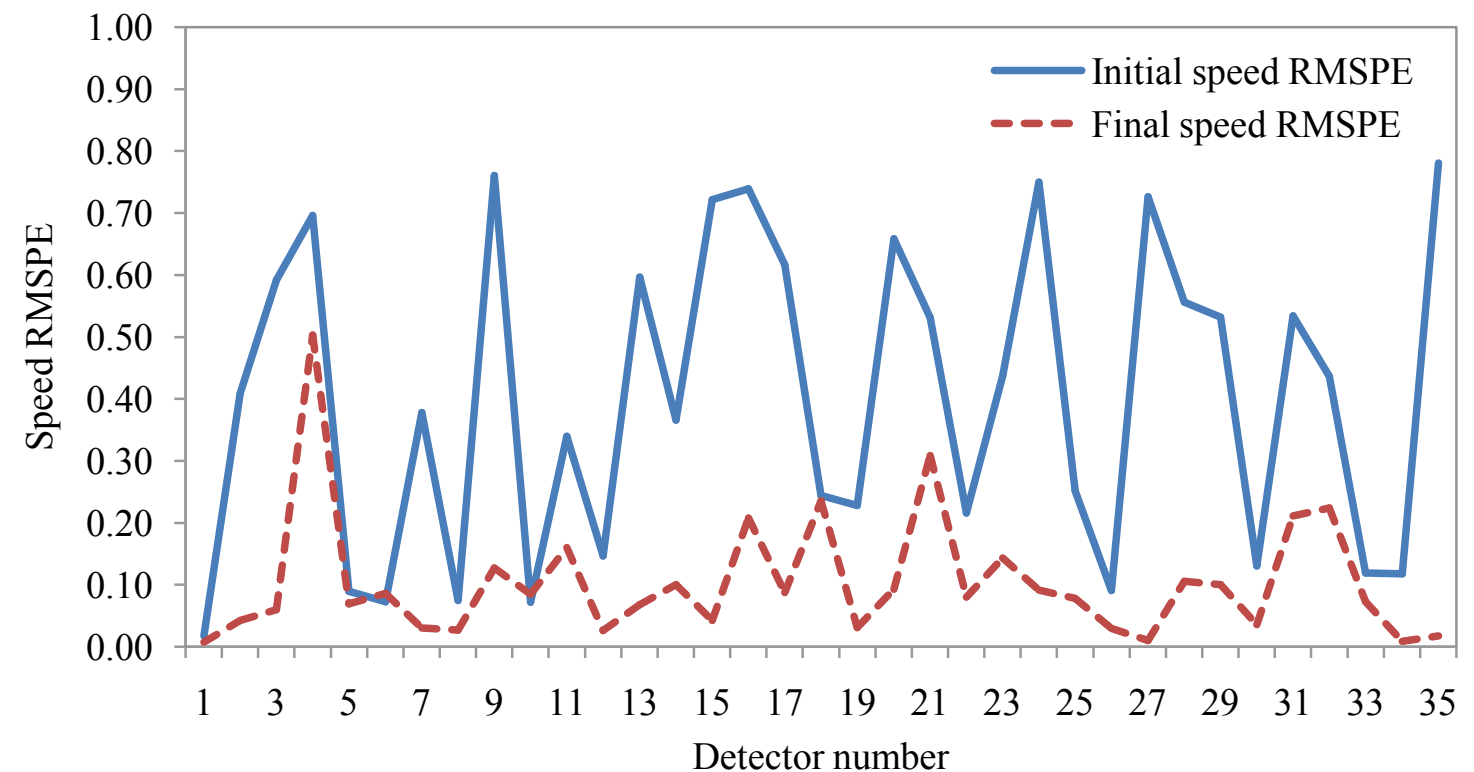

Figure 6.12 Comparison between assignment speed before-and-after applying the proposed methodology framework.

In this case study, the proposed traffic flow model fine-tuning (TFMFT) model is not used since the stochastic optimization algorithm (sequential SPSA) requires simulation hundreds of times to reach a satisfying result. The large size of the network in this case study causes a much longer computation time. Thus, it is unrealistic to use the TFMFT model to calibrate the parameters of the traffic flow model.

Finally, Zhou's dynamic O-D matrix estimation model is used to perform the estimation for this case study. For the purpose of comparison, the initial O-D matrix is directly extracted from the SERPM model and factorized by the volume proportional factor. The traffic flow models are presented in Table 6.1. The performance of Zhou's estimation model is illustrated in Figure 6.13. 


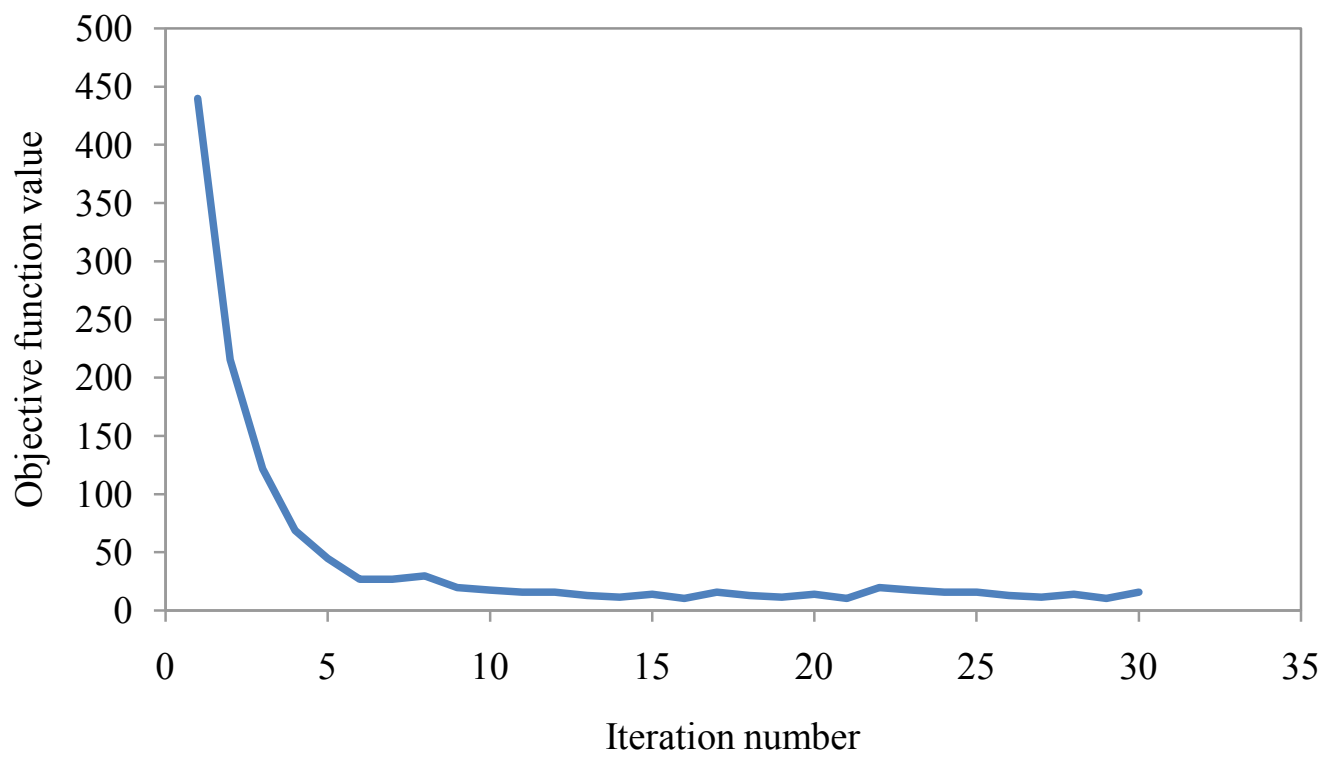

Figure 6.13 Performance of Zhou's dynamic O-D matrix estimation model.

According to Figure 6.13, after 10 initial iterations, the objective function value drops from 450.213 to 10.491 . In subsequent iterations, however, the objective function value stays the same. With the estimated O-D matrix from Zhou's estimation model loading onto the road network, the average RMSPE for volume is 0.168 and 0.179 for average speed RMSPE. Comparatively, in the proposed estimation framework, with the negligible computation time spent by the SIODE, it takes six iterations for the DIODE to reach a stable solution, as indicated by Figures 6.4 and 6.5. Furthermore, it takes 10 iterations for the DODE to reach a stable solution, as indicated by Figure 6.8. The total iteration number for the proposed methodology framework is 16 . Its estimation results have the average volume RMSPE of 0.045 and speed RMSPE of 0.110. Based on the above comparison, it can be concluded that the proposed methodology has a better performance than Zhou's model. 


\subsection{Summary}

In this chapter, the proposed methodology framework is applied to the regional road network in Jacksonville, FL. Before the application of the proposed models, there are two modifications to the methodology: (1) the capacity constraint is enforced to relieve the under-specification problem, and (2) the TFMFT model is not used due to the heavy computation burden. Through estimation, the objective function value drops from 403.145 to 0.7 in 16 iterations. The average RMSPE for the assignment volumes is around 0.045 after the estimation. For the purpose of comparison, Zhou's dynamic O-D matrix estimation model is also used to perform the estimation. The result shows that the objective function value drops from 450.213 to 10.491 in 10 iterations and will not decrease further. The average RMSPE for volume is 0.168 after the estimation by Zhou's model. Based on the data, it can be concluded that the proposed estimation framework shows an improved performance when compared with Zhou's estimation model. Although the average RMSPE for speed was reduced significantly after the dynamic O-D matrix estimation, it is still high. Future efforts should be aim at reducing the RMSPE for speed. 


\section{CHAPTER 7}

\section{RESEARCH SUMMARY}

This study develops a dynamic O-D matrix estimation framework, which has better performance than existing models, especially under congested traffic conditions. This chapter provides conclusions about the performance of each model in the proposed framework, based on the case studies, and restates the original contribution of the proposed framework.

\subsection{Conclusions}

Compared with existing dynamic O-D estimation models, the proposed methodology framework has two advantages: (1) it can produce an initial O-D matrix with a highconfidence level, which has the potential to significantly improve the accuracy of dynamic O-D estimation, and reduce the associated computation time; (2) the framework can automatically convert traffic volume deviation to traffic density deviation in the objective function, under the congested traffic condition, to avoid the side impact from the proportional assignment assumption of the existing dynamic O-D estimation models. In addition, by converting traffic volume data into traffic density data, traffic speed data are implicitly incorporated into the estimation model, which means more traffic information can be incorporated into the estimation model, thus contributing to the improvement in estimation performance.

The proposed methodology framework includes four models: the traffic flow model calibration (TFMC) model, the initial O-D matrix estimation (IODE) model, the dynamic O-D matrix estimation (DODE) model, and the traffic flow model fine-tuning (TFMFT) model. 
The IODE model aims to provide an accurate initial O-D matrix for the proposed DODE model. The advantages of the IODE are, (1) it can reduce the systematic deviation of the link traffic measurement while maintaining the information of the input O-D matrix in terms of relative relationship of O-D demand value; (2) it can relieve the problematic proportional assignment assumption by automatically converting the traffic volume deviation into traffic density deviation in the objective function, under congested traffic conditions; and (3) it can be solved without a heavy computational burden in that less variables are involved.

The DODE model has three advantages over the existing estimation model. First, similar to the IODE model, the DODE model can relieve the problematic proportional assignment assumption by automatically converting the traffic volume deviation into traffic density deviation in the objective function, under congested traffic conditions. Second, it can adaptively update the weighting factor to restrict the magnitude of O-D demand adjustment to avoid the divergent problem. Finally, the DODE model can incorporate the temporal pattern of the historic O-D matrix into the estimation model to further improve estimation.

Three case studies were performed to test the proposed method. In the first case study, a hypothetical network is used to test the DODE model under congested, initial traffic conditions. The DODE model demonstrates much better performance than that of Zhou's model, reaching a good estimation result within eight iterations, while Zhou's estimation model could not obtain a convergent result at all.

In the second case study, the road network is a segment of I-95 in Miami-Dade County, FL. There are a total of 21 zones in the network. It takes seven iterations with the 
proposed method to reach a convergent objective function value of 0.025 , while it takes about 50 iterations with Zhou's estimation model to reach an objective function value of 0.250. After the estimation from the proposed models, the average RMSPE for volume is 0.010 , and the average RMSPE for speed is 0.283 . In comparison, when Zhou's model is used, the final average RMSPE for volume is 0.023 , and the average RMSPE for speed is 0.285 . The case study demonstrates that the proposed method has better performance than Zhou's model. In addition, the TFMFT model can reduce the average RMSPE for speed from 0.283 to 0.130 .

In the third case study, the road network is the regional road network in Jacksonville, FL. There are a total of 149 TAZs in the network. It takes 16 iterations for the proposed method to reach the objective function value of 0.70 , compared to the 10 iterations for Zhou's estimation model to reach the final value of 10.71. Using the proposed method, the final average RMSPE for volume is 0.045 , and the average RMSPE for speed is 0.110 . When Zhou's estimation model is used, the final average RMSPE for volume is 0.168 , and the final average RMSPE for speed is 0.179 . The case study shows that the proposed method is more efficient than Zhou's model. The advantages of the proposed methodology framework in all three case studies demonstrate the feasibility of its application to a large network with an efficient estimation.

\subsection{Limitations and Future Work}

There are several limitations in the proposed method, which need to be addressed in future studies. Firstly, the IODE and DODE models cannot perform O-D matrix estimation on O-D pairs with a zero initial demand value. The existing O-D matrix estimation (dynamic or static) model has the same problem. The reason for this difficulty 
is that the estimation is based on the link proportion matrix $\mathbf{P}$, and $\mathbf{P}$ is generated based on the assignment results from the initial O-D demand matrix. If an O-D pair in the initial O-D matrix has zero demand value, then there is no corresponding contribution factor in P from this O-D pair to link volume. Consequently, the estimation model will not adjust the demand of the O-D pair. For this reason, the proposed framework fails to guarantee a good estimation for the skewed initial O-D demand matrix with multiple zero (missing) demand value. In the future, efforts should be focused on how to combine more information, such as a survey of travelers' behaviors, into the link proportion matrix $\mathbf{P}$, so that the proposed framework has a robust performance given conditions of inaccurate or missing values in the initial O-D matrix.

Secondly, the IODE and DODE models yield poor estimation results when the detectors are located downstream of the simulated traffic bottlenecks. In this situation, the information collected from the detectors fails to indicate the congested situation of the road network because the traffic downstream of the bottlenecks remains uncongested. The estimation models may blindly increase traffic demand to raise the traffic volume in the downstream, which may only weaken the estimation. In the future, the impact of the location and number of detectors needs to be studied to determine the minimum traffic measurement information required to obtain good estimation.

Thirdly, the TFMFT model is inapplicable of dealing with a large road network due to the heavy computational burden. It is a topic for future studies to devise an efficient algorithm to solve this problem.

Finally, because of the under-specification problem, it is problematic to believe that a strong O-D matrix estimate is achieved only based on the matching criteria 
between the assignment traffic measurement data from DTA simulators and the observed traffic measurement data from the same limited detectors. In the future, efforts should be focused on how to effectively combine more traffic measurement data, such as AVI data, into the estimation model to increase accuracy of the estimation results. 


\section{REFERENCES}

Antoniou, C., M. Ben-Akiva, and H. Koutsopoulos (2004). "Incorporating Automated Vehicle Identification Data into Origin-Destination Estimation." Transportation Research Record: Journal of the Transportation Research Board, No. 1882, pp. 37-44.

Antoniou, C., M. Ben-Akiva and H.N. Koutsopoulos (2007). "Nonlinear Kalman Filtering Algorithms for On-Line Calibration of Dynamic Traffic Assignment Models." IEEE Transportations on Intelligent Transportation, Vol. 8, No. 4, pp. 661-670.

Ashok, K. and M.E. Ben-Akiva (1993). "Dynamic Origin-Destination Matrix Estimation for Real-Time Traffic Management Systems." Proceedings of the $12^{\text {th }}$ International Symposium on the Theroy of Traffic Flow and Transportation. (Daganzo, C.F. ed.), Elsevier Science Publishing Company, Inc. Berkeley, CA, USA, pp. 465-484.

Ashok, K. and M.E. Ben-Akiva (2000). "Alternative Approaches for Real-Time Estimation and Prediction of Time-Dependent Origin-Destination Flows." Transportation Science, Vol.36, No.2, 2002. pp 184-198.

Ashok, K. and M.E. Ben-Akiva (2002). "Estimation and Prediction of Time-Dependent Origin-Destination Flows with a Stochastic Mapping to Path Flows and Link Flows". Transportation Science, Vol.36, No.2, pp 184-198.

Balakrishna, R., C. Antoniou, M.E. Ben-Akiva, H.N. Koutsopoulos and Y. Wen (2007). "Calibration of Microscopic Traffic Simulation Models: Methods and Application." Transportation Research Record: Journal of the Transportation Research Board, No. 1999, pp. 198-207.

Balakrishna, R., M.E. Ben-Akiva and H.N. Koutsopoulos (2007). "Off-line Calibration of Dynamic Traffic Assignment: Simultaneous Demand-Supply Estimation." Transportation Research Record: Journal of the Transportation Research Board, No. 2003, pp. 50-58.

Bell, M.G.H. (1991a). "The Estimation of Origin-Destination Matrices by Constrained Generalized Least Squares.” Transportation Research Part B: Methodological, Vol. 25B, No. 1, pp. 13-22.

Bell, M.G.H. (1991b). "The Real Time Estimation of Origin-Destination flows in the Presence of Platoon Dispersion." Transportation Research Part B: Methodological, Vol. 25B, No. 2/3, pp. 115-125.

Bell, M.G.H. and Y. Iida (1997). Transportation Network Analysis, John Wiley \& Sons.

Bierlaire, M. and F. Crittin (2004). "An Efficient Algorithm for Real-Time Estimation and Prediction of Dynamic OD Tables." Operations Research, Vol. 52, No. 1, pp. 116127. 
Cascetta, E. (1984). "Estimation of Trip Matrices from Traffic Counts and Survey Data: A Generalized Least Squares Estimator." Transportation Research Part B: Methodological, Vol. 18B, No.4/5, pp. 289-299.

Cascetta, E. and S. Nguyen (1988). "A Unified Framework for Estimating or Updating Origin/Destination Matrices from Traffic Counts." Transportation Research Part B: Methodological, Vol. 22, No. 6, pp. 437-455.

Cascetta, E., D. Inaudi, and G. Marquis (1993). "Dynamic Estimators of OriginDestination Matrices Using Traffic Counts." Transportation Science, Vol. 27, No. 4, pp. 363-373.

Cascetta, E. and M. N. Postorino (2001). "Fixed Point Approaches to the Estimation of O/D Matrices Using Traffic Counts on Congested Networks." Transportation Science, Vol. 35, No. 2, pp. 134-147.

Chang, G. L. and J. Wu (1994). "Recursive Estimation of Time-Varying OriginDestination Flows from Traffic Counts in Freeway Corridors." Transportation Research Part B: Methodological, Vol. 28, No. 2, pp. 141-160.

Caliper (2000). TransCAD User's Guide Version 4.5. Caliper Corp., Newton, MA.

Casey, H.J (1995). "Applications to Traffic Engineering of the Law of Retail Gravitation." Traffic Quarterly IX, pp.23-25.

Citilabs (2004). Cube User's Guide Version 3.2.1. Citilabs, Inc., Oakland, CA.

Cremer, M. and H. Keller (1981). "Dynamic Identification of Flows from Traffic Counts at Complex Intersections." Proceedings the $8^{\text {th }}$ International Symposium on Transportation and Traffic Theory, (V. F. Hurdle, V.F et al Eds.) Toronto University Press, Toronto, ON, Canada, pp. 199-209.

Cremer M. and H. Keller (1984). "A Systems Dynamics Approach to the Estimation of Entry and Exit O-D Flows." Proceedings of the $9^{\text {th }}$ International Symposium on Transportation and Traffic Theory. (Volmuller, I. and R. Hamerslag eds.) VNU Science Press, Utrecht, the Netherlands, pp. 431-450.

Cremer M. and H. Keller (1987). "A New Class of Dynamic Methods for Identification of Origin-Destination Flows." Transportation Research Part B: Methodological, Vol. 21B, No. 2, pp. 117-132.

Dixon, M. P. (2000). Incorporation of Automatic Vehicle Identification Data into the Synthetic OD Estimation Process. P.hD. Dissertation. Department of Civil Engineering, Texas A\&M University, College Station. TX. 
Dixon, M. P. and L.R. Rilett (2002). "Real-Time OD Estimation Using Automatic Vehicle Identification and Traffic Count Data". Computer-Aided Civil and Infrastructure Engineering, Vol. 17, pp. 7-21.

Gajewski, B.J., L.R. Rilett, M.P. Dixon, and C.H. Spiegelman (2002). "Robust Estimation of Origin-Destination Matrices." Journal of Transportation and Statistics. Vol. 5, No. 2/3, pp 37-56.

Kalman, R. E. (1960). "A New Approach to Linear Filtering and Prediction Problems." Transactions of the ASME - Journal of Basic Engineering, Vol. 82, pp. 35-45.

Kattan, L. and B. Abdulhai (2006). "Noniterative Approach to Dynamic Traffic OriginDestination Estimation with Parallel Evolutionary Algorithms." Transportation Research Record: Journal of the Transportation Research Board, No. 1964, pp. 201-210.

Kim, S.J. (2006). Simultaneous Calibration of a Microscopic Traffic Simulation Model and $O D$ Matrix. P.hD. Dissertation. Department of Civil Engineering, Texas A\&M University, College Station. TX.

Kuwahara, M. and E.C. Sullivan (1987). "Estimating O-D Matrices from Roadside Survey Data". Transportation Research Part B: Methodological, Vol. 21B, No. 3, pp. 233-248.

Kwon, J. and P.Varaiya (2005). "Real-Time Estimation of Origin-Destination Matrices with Partial Trajectories from Electronic Toll Collection Tag Data". Compendium of papers, the 84th Annual Meeting of the Transportation Research Board, National Research Council, Washington, DC.

Lin, W. (2006). A Robust Model for Estimating Freeway Dynamic Origin-Destination Matrices. PhD. Dissertation, Department of Civil and Environmental Engineering University of Maryland, College Park. MD.

Maher, M. J. (1983). "Inferences on Trip Matrices from Observations on Link Volumes: A Bayesian Statistical Approach." Transportation Research Part B: Methodological, Vol. 17B, No. 6, pp. 435-447.

Mahmassani, H. S., H. Sbayti and X. Zhou (2005). DYNASMART-P, User's Guide Version 1.2. Maryland Transportation Initiative, University of Maryland, College Park, MD.

Mahmassani, H.S., X. Qin and X. Zhou (2004). DYNASMART-X Evaluation for RealTime TMC Application: Irvine Test Bed. US DOT/FHWATREPS Phase 1.5B final report, Maryland Transportation Initiative, University of Maryland.

Murtagh, B.A. and M. A. Saunders (1987), MINOS 5.1 User`s Guide, Report SOL 8320R, Department of Operations Research, Stanford University, 
Nie, Y., H.M. Zhang and W.W. Recker (2005). "Inferring origin-destination trip matrices with a decoupled GLS path flow estimator." Transportation Research Part B: Methodological, Vol. 39, pp. 497-518.

Nie, Y. and H.M. Zhang (2008). "Relaxation Approach for Estimating OriginDestination Trip Tables." Networks and Spatial Economics, Vol. 10, pp. 147-172.

Nie, Y. and H.M. Zhang (2008). "A Variational Inequality Approach for Inferring Dynamic Origin-Destination Travel Demands." Transportation Research Part B: Methodological, Vol. 42, pp. 635-662.

Nihan, N.L. and G.A. Davis (1987). "Recursive Estimation of Origin Destination Matrices from Input/Output Counts." Transportation Research Part B: Methodological, Vol. 21B, No. 2, pp. 149-163.

Okutani, I. (1987). "The Kalman Filtering Approach in Some Transportation and Traffic Problems." Proceedings of the $10^{\text {th }}$ International Symposium on Transportation and Traffic Theory (N.H. Gartner and N.H.M. Wilson, eds.), Elsevier Science Publishing Company, Inc. Cambridge, MA, pp. 397-416.

Schrank, D.L. and T.J. Lomax (2009). The 2009 Urban Mobility Report. Texas Transportation Institute, Texas A\&M University, College Station, TX.

Sherali, H. D. and T. Park (2001) "Estimation of Dynamic Origin-Destination Trip Tables for a General Network." Transportation Research Part B: Methodological, Vol. 35B, pp. 217-235.

Spall, J.C. (1998). "Implementation of the Simultaneous Perturbation Algorithm for Stochastic Optimization." IEEE Transactions on Aerospace and Electronic Systems, Vol. 34, pp.47 817-823.

Spiess, H. (1987). "A Maximum Likelihood Model for Estimating Origin-Destination Matrices." Transportation Research Part B: Methodological, Vol. 21B, No. 5, pp. 395412.

Sun, C. and H. Porwal (2000). "Dynamic Origin/Destination Estimation Using True Section Densities." Research Reports: Paper UCB-ITS-PRR-2000-5, California Partners for Advanced Transit and Highways (PATH), University of California, Berkeley CA.

Tavana, H. and H.S. Mahmassani (2001). "Estimation of Dynamic Origin-Destination Flows from Sensor Data Using Bi-Level Optimization Method." Compendium of Papers, the 80th Annual Meeting of the Transportation Research Board, National Research Council, Washington, DC. 
Van Zuylen, H. (1978). "The Information Minimizing Method: Validity and Applicability to Transport Planning." New Developments in Modeling Travel Demand and Urban Systems (Jansen, G. R. M. et al. eds.). Aaxon, Famborough.

Van Zuylen, H.J. and L.G. Willumsen (1980). "The Most Likely Trip Matrix Estimated from Traffic Counts." Transportation Research Part B: Methodological, Vol. 14B, pp. 281-293.

Van der Zijpp, N.J. (1997). "Dynamic OD-Matrix Estimation from Traffic Counts and Automated Vehicle Identification Data." Transportation Research Record: Journal of the Transportation Research Board, No. 1607, pp. 87-94.

Wilson A. G. (1970), Entropy in Urban and Regional Modeling. Pion, London.

Zhou, X., X. Qin and H.S. Mahmassani (2003). "Dynamic Origin-Destination Demand Estimation Using Multi-Day Link Traffic Counts for Planning Applications." Transportation Research Record: Journal of the Transportation Research Board, No. 1831, pp. 30-38.

Zhou, X. and H.S. Mahmassani (2005). "Recursive Approaches for Online Consistency Checking and OD Demand Updating for Real-time Dynamic Traffic Assignment Operation." Transportation Research Record: Journal of the Transportation Research Board, No. 1923, pp. 218-226.

Zhou, X. and H.S. Mahmassani (2006). "Dynamic OD Estimation Using Automatic Vehicle Identification Data." IEEE Transactions on Intelligent Transportation Systems, Vol. 7, No. 1, pp. 105-114.

Zhou, X., H.S. Mahmassani (2007). "A Structural State Space Model for Real-Time Traffic Origin-Destination Demand Estimation and Prediction in A Day-to-Day Learning Framework." Transportation Research Part B: Methodological, Vol. 41B, pp. 823-840. 
VITA

\section{HONGBO CHI}

Jan. 1979

Born, Inner Mongolia, China

\section{EDUCATION}

$1996-2001$

B.S., Civil Engineering

Beijing University of Technology

Beijing, China

$2001-2004 \quad$ M.S., Civil Engineering

Beijing University of Technology

Beijing, China

$2004-2010 \quad$ Doctoral Candidate in Civil Engineering

Florida International University

Miami, Florida

\section{EMPLOYMENT}

2005 - 2009 Graduate Research Assistant

Lehman Center for Transportation Research

Florida International University

Miami, Florida

\section{AFFILIATIONS}

- $\quad$ Student member, Chi Epsilon Honor Society, 2005 - Present

- $\quad$ Student member, Institute of Transportation Engineers, 2004 - Present

\section{PUBLICATIONS AND PRESENTATIONS}

- Chi, H., F. Zhao, and X. Sun. (2010), “An Effective Dynamic Origin-Destination Matrix Estimation Framework". Compendium of Papers, the 89th Annual Meeting of the Transportation Research Board, National Research Council, Washington, D.C.

- $\quad$ Chow, L., H. Chi and F. Zhao. (2010), "Subregional Transit Ridership Models Based on Geographically Weighted Regression". Compendium of Papers, the 89th Annual Meeting of the Transportation Research Board, National Research Council.

- $\quad$ Chi, H., C. Lu, and F. Zhao. (2009), "Vehicle Detection from Satellite Images: Intensity, Chromaticity-, and Lane-Based Method". Transportation Research Record, Journal of the Transportation Research Board, No. 2105, Transportation Research Board, National 
Research Council, Washington, D.C., 2009, pp. 109-117.

- Zhao, F., H. Chi, H. Qiang, K. Xing, and L. D. Shen (2008). Use of Satellite Imagery for Post Hurricane Traffic Condition Assessment, Report USDOT-FTA-FL-BDF84, Lehman Center for Transportation Research, Florida International University, Miami, FL.

- $\quad$ Chi, H., F. Zhao, and D. Shen. (2008), "Delineating Travel Lanes from Satellite Images for Disaster Relief Application". The Fourteenth ISSAT International Conference on Reliability and Quality in Design, pp. 240-243.

- Chi, H., F. Zhao, L. Chow and D. Shen. (2008). "Estimating Evacuation Impact on Transportation Network with Dynamic Traffic Assignment". The Eighth International Conference of Chinese Transportation and Logistics Professionals.

- Zhao, F., and H. Chi. (2007). A Rapid Transportation Network Condition Assessment System for Intermodal Security and Safety, Report FTA-FL-26-7102-01, Lehman Center for Transportation Research, Florida International University, Miami, FL.

\section{HONORS AND AWARDS}

- $\quad$ Dissertation Year fellowship, Florida International University, 2009-2010.

- Fourth place Spring 2010 ITE Gold Coast Chapter Book Scholarship Award for the paper titled "Stepwise GWR-Based Transit Ridership Estimation Model." ITE Gold Coast Chapter, 2010.

- $\quad$ AAHTO Franncios Award, 2009.

- $\quad$ Grand Prize, Student Poster Competition, FIU GIS Day, March 2009.

- $\quad$ Second Prize, Gold Coast Chapter Book Scholarship Essay Competition January 2008.

- $\quad$ Presidential Enhanced Fellowship, Florida International University, 2004-2008. 